\title{
A new checklist of lichenized fungi occurring in Thailand
}

Kawinnat Buaruang', Kansri Boonpragob', Pachara Mongkolsuk', Ek Sangvichien', Kajohnsak Vongshewarat', Wetchasart Polyiam', Achariya Rangsiruji ${ }^{2}$, Wanaruk Saipunkaew ${ }^{3}$, Khwanruan Naksuwankul ${ }^{4}$, Jutarat Kalb ${ }^{5}$, Sittiporn Parnmen ${ }^{6}$, Ekaphan Kraichak ${ }^{7}$, Phimpisa Phraphuchamnong', Sanya Meesim', Theerapat Luangsuphabool', Phimpha Nirongbut', Vasun Poengsungnoen', Natwida Duangphui', Mattika Sodamuk', Supatra Phokaeo', Muthita Molsil', André Aptroot ${ }^{8}$, Klaus Kalb ${ }^{9}$, Robert Lücking ${ }^{10}$, H. Thorsten Lumbsch"1

I Lichen Research Unit, Biology Department, Faculty of Science, Ramkhamhaeng University, Ramkhamhaeng 24 road, Bangkok, 10240 Thailand 2 Department of Biology, Faculty of Science, Srinakharinwirot University, Bangkok, Thailand 3 Department of Biology, Faculty of Science, Chiang Mai University, 239 Huay Kaew Rd., Suthep, Mueang, Chiang Mai, 50200 Thailand 4 Department of Biology and Natural Medicinal Mushroom Museum, Faculty of Science, Mahasarakham University, Kantarawichai, Maha Sarakham Province, 44150 Thailand 5 King Mongkut's Institute of Technology Ladkrabang, Prince of Chumphon campus, 17/1 Moo 6, Chumcho, Patieu district, Chumphon, 86160 Thailand 6 Toxicology center, National Institute of Health, Department of Medical Sciences, Ministry of Public Health, Tivanon Rd., Nonthaburi 11000, Thailand 7 Department of Botany, Faculty of Science, Kasetsart University, Bangkok 10900, Thailand 8 ABL Herbarium, Gerrit van der Veenstraat 107, NL-3762 XK Soest, The Netherlands 9 Lichenologisches Institut Neumarkt, Im Tal 12, D-92318 Neumarkt, Germany, and University of Regensburg, Institute of Plant Sciences, Universitätsstraße 31, D-93040 Regensburg, Germanys 10 Botanischer Garten und Botanisches Museum Berlin, Königin-Luise-Strasse 6-8, 14195 Berlin, Germany I I Science \& Education, The Field Museum, 1400 South Lake Shore Drive, Chicago, Illinois 60605-2496, U.S.A.

Corresponding author: H. Thorsten Lumbsch (tlumbsch@fieldmuseum.org)

Academic editor: C. Gueidan | Received 11 March 2017 | Accepted 13 April 2017 | Published 9 June 2017

Citation: Buaruang K, Boonpragob K, Mongkolsuk P, Sangvichien E, Vongshewarat K, Polyiam W, Rangsiruji A, Saipunkaew W, Naksuwankul K, Kalb J, Parnmen S, Kraichak E, Phraphuchamnong P, Meesim S, Luangsuphabool T, Nirongbut P, Poengsungnoen V, Duangphui N, Sodamuk M, Phokaeo S, Molsil M, Aptroot A, Kalb K, Lücking R, Lumbsch HT (2017) A new checklist of lichenized fungi occurring in Thailand. MycoKeys 23: 1-91. https://doi. org/10.3897/mycokeys.23.12666

Copyright Kawinnat Buaruang et al. This is an open access article distributed under the terms of the Creative Commons Attribution License (CC BY 4.0), which permits unrestricted use, distribution, and reproduction in any medium, provided the original author and source are credited. 


\begin{abstract}
A new revised checklist of lichenized fungi in Thailand is presented, including 1,292 species. Recent work on the taxonomy of these organisms in Thailand resulted in an enormous increase in our knowledge of the lichen biota of the country - the current checklist includes more than twice as many species as the previous catalogue published 15 years ago - and phylogenetic studies resulted in numerous changes in the generic classification of lichenized fungi. Hence, a new checklist is here presented summarizing the current knowledge of lichens in Thailand. Six new records are reported, viz. Acanthothecis salazinica, Bactrospora metabola, Buellia parastata, Diploschistes cinereocaesius, Rolfidium coccocarpioides, and Trapelia placodioides. Five previously recorded species, namely Lecanora carpinea, Platismatia glauca, P. Lacunosa, P. tuckermanii and Roccella phycopsis are shown to be based on misidentifications and are excluded from the checklist. Three new combinations of species previously placed in Pertusaria to Lepra are proposed: L. bulolensis (A.W.Archer, Elix \& Streimann) Schmitt \& Lumbsch, L. patellifera (A.W.Archer) Schmitt \& Lumbsch, and L. subventosa (Malme) Schmitt \& Lumbsch.
\end{abstract}

\title{
Keywords
}

Asia, biodiversity, lichens, new records, taxonomy

\section{Introduction}

The lichen flora of Thailand is rich due to the numerous habitats with high diversity especially in montane forests and coastal forests and the fact that it includes monsoonal, seasonal dry habitats, tropical lowland forests in the south and subtropical habitats in northern montane forests. The country is situated in the Indo-Chinese floristic region, bordering the distinct Indian, Malesian and the Eastern Asiatic floristic regions (Takhtajan 1986) with floristic interchange adding to the diversity of the flora. Despite the species richness of this tropical country, the lichen flora has been poorly known for a long time. This is partly due to the fact that the exploration of the lichen flora depended almost entirely on sporadic visits of overseas scientists starting with studies by Vainio $(1909,1921)$ reporting on collections by Danish expeditions to Koh Chang island in the southeast of the country and Doi Suthep in northern Thailand. A number of workshops and increased collaborations of oversees scientists with Thai colleagues starting in the 1990s laid the foundation for a renewed interest in lichens in the country, including the workshop 'Lichen Taxonomy and Biology' at Khao Yai National Park in 1998 (Boonpragob et al. 1998), a 'Thelotremataceae workshop' in 2008 (Boonpragob et al. 2010), and the organization of the IAL7 conference in 2012 (Brodo 2012). This resulted in an enormous increase in studies addressing lichen biodiversity and taxonomy in Thailand. As a result of this scientific activity the number of species listed in this checklist $(1,292)$ is more than twice as high as in the last catalogue (Wolseley et al. 2002) with 554 species that was only published 15 years ago. In parallel with this increase in knowledge of the diversity of lichens in Thailand, our understanding of the evolution of lichenized fungi and their phylogenetic relationships dramatically increased over the last decades due to molecular data (Jaklitsch et al. 2016; Lücking et al., 2016; Lumbsch 2000; Lumbsch and Huhndorf 2010; Printzen 2010). This resulted in numerous changes in the generic classification that make it difficult for 
users to interpret older names in the literature. To make the information of our current knowledge of species occurring in Thailand and their currently used names more readily available, we have compiled this revised checklist that is based on Wolseley et al. (2002) and subsequently published results and also includes revision of doubtful records. As a result of our studies of voucher material, five species are excluded from the checklist. We anticipate that this checklist will help to further stimulate taxonomic research in Thailand and adjacent countries in Southeast Asia.

\section{The checklist}

1. Absconditella delutula (Nyl.) Coppins \& H.Kilias, in Hawksworth et al., Lichenologist 12: 106 (1980)

Literature: Aptroot et al., 2007

2. Acanthothecis salazinica S. Joshi \& Hur, Lichenologist 45: 600 (2013)

3. Aderkomyces armatus (Vězda) Lücking, Sérus. \& Vězda, Lichenologist 37: 161 (2005)

Literature: Aptroot et al., 2007

4. Aderkomyces thailandicus Papong, Boonpragob \& Lücking, Phytotaxa 18: 11 (2011)

Literature: Lumbsch et al., 2011

5. Agonimia pacifica (H.Harada) Diederich, in Aptroot et al., Biblioth. Lichenol. 64: 12 (1997)

Literature: Aptroot et al., 2007

6. Agonimia tristicula (Nyl.) Zahlbr., Bot. Anstalt. Wien, Österr. bot. Z. 5: 351 (1909)

Literature: Aptroot et al., 2007

7. Amandinea diorista (Nyl.) Marbach, Biblioth. Lichenol. $74: 58$ (2000)

Literature: Meesim and Mongkolsuk 2010b

8. Amandinea diorista var. hypopelidna (Stirt.) Marbach, Biblioth. Lichenol. 74: $60(2000)$

Literature: Van den Boom et al. 2014

9. Amandinea efflorescens (Müll.Arg.) Marbach, Biblioth. Lichenol. 74: 61 (2000) Literature: Aptroot et al., 2007; Van den Boom et al. 2014

10. Amandinea extenuata (Müll.Arg.) Marbach, Biblioth. Lichenol.74: 71 (2000) Literature: Aptroot et al., 2007

11. Amandinea melaxanthella (Nyl.) Marbach, Biblioth. Lichenol. 74: 87 (2000) Literature: Vainio 1909 as Buellia melaxanthella; Wolseley et al., 2002 as Buellia stramineoatra; Aptroot et al., 2007; Van den Boom et al., 2014

12. Amandinea montana (H. Magn.) Marbach, Biblioth. Lichenol. 74: 93 (2000) Literature: Meesim and Mongkolsuk 2010b as Buellia montana

13. Amandinea pelidna (Ach.) Fryday \& L. Arcadia, Graphis scripta 24: 41 (2012) Literature: Mongkolsuk and Meesim 2012, as Amandinea lecideina 
14. Amandinea placodiomorpha (Vain.) Marbach, Biblioth. Lichenol. 74: 99 (2000)

Literature: Aptroot et al., 2007

15. Amandinea punctata (Hoffm.) Coppins \& Scheid., Lichenologist 25: 343 (1993)

Literature: Meesim and Mongkolsuk 2010b as Buellia punctata; Mongkolsuk and Meesim 2012

16. Ampliotrema palaeoamplius (Aptroot \& Sipman) Kalb ex Kalb, Biblioth. Lichenol. 92: 81 (2006)

Literature: Yoshimura 1978; Homchantara and Coppins 2002; Wolseley et al., 2002 as Ocellularia palaeoamplior; Kalb 2009

17. Ampliotrema rimosum (Hale) Mercado-Díaz, Lücking \& Parnmen, Phytotaxa 189: 190 (2014)

Literature: Kraichak et al. 2014; Lumbsch et al., 2014 as Ocellularia rimosa; Sutjaritturakan and Kalb 2015

18. Anisomeridium adnexum (Müll.Arg.) R.C.Harris, More Florida Lichens, Incl. 10 Cent Tour Pyrenol.: 143 (1995)

Literature: Aptroot et al., 2007

19. Anisomeridium asiaticum Vain., Hedwigia 46: 180 (1907)

Literature: Vainio 1909; Alava 1988; Wolseley et al., 2002 as Microthelia asiatica

20. Anisomeridium consimile (Vain.) R.C.Harris, More Florida Lichens, Incl. 10 Cent Tour Pyrenol. (New York): 145 (1995)

Literature: Aptroot et al., 2007

21. Anisomeridium consobrinum (Nyl.) Aptroot, in Aptroot et al., Biblioth. Lichenol. 57: 21 (1995)

Literature: Mongkolsuk et al., 2013 as Arthopyrenia consobrina

22. Anisomeridium foliicola R.Sant. \& Tibell, Austrobaileya 2: 531 (1988)

Literature: Boonpragob et al., 1998; Wolseley et al., 2002

23. Anisomeridium polypori (Ellis \& Everh.) M.E.Barr, in Barr et al., Mem. New York Bot. Gard. 79: 76 (1996)

Literature: Aptroot et al., 2007

24. Anisomeridium subprostans (Nyl.) R.C.Harris, in Tucker \& Harris, Bryologist 83: 4 (1980)

Literature: Aptroot et al., 2007

25. Anisomeridium tamarindi (Fée) R.C.Harris, in Tucker \& Harris, Bryologist 83: 4 (1980)

Literature: Aptroot et al., 2007

26. Anisomeridium throwerae R.C.Harris, More Florida Lichens, Incl. 10 Cent Tour Pyrenol.: 150 (1995)

Literature: Schumm and Aptroot 2012

27. Anthracothecium macrosporum (Hepp) Müll.Arg., Linnaea 43: 44 (1880)

Literature: Paulson 1930 as Anthracothecium pyrenuloides; Wolseley et al., 2002; Aptroot et al., 2007 as Anthracothecium columellatum 
28. Anthracothecium prasinum (Eschw.) R.C.Harris, in Egan, Bryologist 90: 163 (1987)

Literature: Wolseley and Aguirre-Hudson 1997a, 1997b; Boonpragob et al., 1998; Wolseley et al., 2002

29. Arctomia fascicularis (L.) Otálora \& Wedin, Lichenologist 45: 292 (2013)

Literature: Aptroot et al., 2007 as Collema fascicularis

30. Arthonia complanata Fée, Essai Crypt. Écorc.: 54 (1825) [1824]

Literature: Vainio 1909; Wolseley et al., 2002

31. Arthonia cyanea var. cyanea Müll.Arg., Flora 64: 233 (1881)

Literature: Papong et al., 2007

32. Arthonia lividula Vain., Ann. Acad. Sci. Fenn., Ser. A, 15: 309 (1921)

Literature: Papong et al., 2007

33. Arthonia mira R.Sant., Symb. Bot. Upsal. 12: 77 (1952)

Literature: Boonpragob et al., 1998; Wolseley et al., 2002

34. Arthonia obscuratula Zahlbr., Cat. Lich. Univ. 2: 71 (1922) [1924]

Literature: Vainio 1909; Wolseley et al., 2002 as Arthonia obscurata

35. Arthonia ochrodes Nyl., Syn. Gen. Arthonia: 10 (1890)

Literature: Vainio 1909; Wolseley et al., 2002

36. Arthonia perpallens Nyl., Ann. Sci. Nat., Bot. 20: 231 (1863)

Literature: Vainio 1909; Wolseley et al., 2002

37. Arthonia ramosii (Räsänen) R.Sant., Symb. Bot. Upsal. 12: 76 (1952)

Literature: Papong et al., 2007

38. Arthonia rhizophorae Vain., Bot. Tidsskr. 29: 141 (1909)

Literature: Vainio 1909; Alava 1988; Wolseley et al., 2002

39. Arthonia trilocularis Müll.Arg., Flora 64: 233 (1881)

Literature: Papong et al., 2007

40. Arthonia varia var. stenographella (Nyl.) Vain., Ann. Acad. Sci. Fenn., Ser. A, 15: 393 (1921)

Literature: Vainio 1909 as Arthonia stenographella; Wolseley et al., 2002

41. Arthothelium monosporum (Vain.) Zahlbr., Cat. Lich. Univ. 2: 129 (1922) [1924]

Literature: Vainio 1921; Alava 1988 as Arthonia monospora; Wolseley et al., 2002

42. Arthothelium ruanum (A.Massal.) Körb., Parerga Lichenol.: 263 (1861)

Literature: Vainio 1909 as Arthonia ruana; Wolseley et al., 2002

43. Arthothelium ruanum var. phaeosporum (Vain.) Zahlbr., Cat. Lich. Univ. 2: 132 (1922)

Literature: Vainio 1909; Alava 1988 as Arthonia ruana var. phaeospora; Wolseley et al., 2002

44. Arthothelium spectabile A.Massal., Ric. Auton. Lich. Crost.: 54 (1852)

Literature: Paulson 1930 as Arthothelium spectabile; Wolseley et al., 2002

45. Aspidothelium cinerascens Vain., Acta Soc. Fauna Fl. Fenn. 7: 189 (1890)

Literature: Aptroot et al., 2007 
46. Aspidothelium fugiens (Müll.Arg.) R.Sant., in Thorold, J. Ecol. 40: 129 (1952) Literature: Aptroot et al., 2007

47. Asteristion alboannuliforme (Nagarkar, Sethy \& Patw.) I. Medeiros, Lücking \& Lumbsch, Fieldiana 9: 7 (2017)

Literature: Wolseley et al., 2002 as Thelotrema alboannuliforme

48. Asterothyrium microsporum R.Sant., Symb. Bot. Upsal. 12: 320 (1952)

Literature: Aptroot et al., 2007

49. Asterothyrium pittieri Müll.Arg., Bull. Soc. Roy. Bot. Belgique 30: 71 (1891)

Literature: Aptroot et al., 2007

50. Astrochapsa astroidea (Berk. \& Broome) Parnmen et al., PLoS ONE 7 (12): e51392 (2012)

Literature: Boonpragob et al., 1998; Wolseley et al., 2002 as Thelotrema astroideum; Parnmen, et al. 2012 as Chapsa astroidea; Lumbsch et al., 2014; Poengsungnoen et al., 2014a

51. Astrochapsa calathiformis (Vain.) Parnmen et al., PLoS ONE 7 (12): e51392 (2012)

Literature: Vainio 1909; Yoshimura 1978; Wolseley et al., 2002 as Thelotrema calathiforme; Homchantara and Coppins 2002 as Thelotrema phliuense; Papong et al., 2010 as Chapsa calathiformis

52. Astrochapsa kalbii Poengs., Lücking \& Lumbsch, Phytotaxa 189: 274(2014)

Literature: Poengsungnoen et al., 2014a

53. Astrochapsa zahlbruckneri (Nyl.) Parnmen, Lücking \& Lumbsch, PLoS ONE 7 (12): e51392, 10 (2012)

Literature: Lumbsch et al., 2014; Poengsungnoen et al., 2014a

54. Astrochapsa pseudophlyctis (Nyl.) Parnmen, Lücking \& Lumbsch, PLoS ONE 7 (12): e51392, 9 (2012)

Literature: Wolseley et al., 2002 as Thelotrema subhiatum; Parnmen, et al. 2012 as Chapsa pseudophlyctis; Lumbsch et al., 2014; Poengsungnoen et al., 2014a

55. Astrothelium aenascens Aptroot, in Aptroot et al. , Lichenologist 48: 611 (2016) Literature: Luangsuphabool et al., 2016

56. Astrothelium aeneum (Eschw.) Aptroot \& Lücking, Lichenologist 48: 821 (2016) Literature: Aptroot et al., 2007 as Trypethelium aeneum; Aptroot and Lücking 2016

57. Astrothelium cinereorosellum (Kremp.) Aptroot \& Lücking, Lichenologist 48: 853 (2016)

Literature: Aptroot et al., 2007 as Trypethelium cinereorosellum; Aptroot and Lücking 2016

58. Astrothelium degenerans (Vain.) Aptroot \& Lücking, Lichenologist 48: 857 (2016)

Literature: Aptroot et al., 2007, as Bathelium degenerans; Aptroot and Lücking 2016

59. Astrothelium eustomum (Mont.) Müll.Arg., Flora 68: 247 (1885)

Literature: Aptroot et al., 2007; Aptroot and Lücking 2016 
60. Astrothelium flavocoronatum Luangsuphabool, Aptroot \& Sangvichien, Lichenologist 48: 731 (2016)

Literature: Luangsuphabool et al., 2016

61. Astrothelium indicum (Upreti \& Ajay Singh) Aptroot \& Lücking, Lichenologist 48: 857 (2016)

Literature: Aptroot et al., 2007, as Laurera indica; Aptroot and Lücking 2016

62. Astrothelium keralense (Upreti \& Ajay Singh) Aptroot \& Lücking, Lichenologist 48: 865 (2016)

Literature: Vongshewarat et al., 1999; Wolseley et al., 2002, both as Laurera keralensis; Aptroot and Lücking 2016

63. Astrothelium macrocarpum (Fée) Aptroot \& Lücking, Lichenologist 48: 867 (2016)

Literature: Aptroot et al., 2007 as Astrothelium galbineum; Aptroot and Lücking 2016

64. Astrothelium macrostiolatum Luangsuphabool, Aptroot \& Sangvichien, Lichenologist 48: 732 (2016)

Literature: Luangsuphabool et al., 2016

65. Astrothelium megaspermum (Mont.) Aptroot \& Lücking, Lichenologist 48: 870 (2016)

Literature: Vongshewarat et al., 1999; Wolseley et al., 2002, both as Laurera megasperma; Aptroot and Lücking 2016

66. Astrothelium meristosporoides (P.M.McCarthy \& Vongshew.) Aptroot \& Lücking, Lichenologist 48: 871 (2016)

Literature: Vongshewarat et al., 1999; Wolseley et al., 2002, both as Laurera meristosporoides; Aptroot and Lücking 2016

67. Astrothelium meristosporum (Mont. \& Bosch) Aptroot \& Lücking, Lichenologist 48: 871 (2016)

Literature: Vainio 1909; Alava 1988 as Thelenella interrupta; Boonpragob et al., 1998; Wolseley et al., 2002, all as Laurera meristospora; Aptroot and Lücking 2016

68. Astrothelium neglectum Luangsuphabool, Aptroot \& Sangvichien, Lichenologist 48: 733 (2016)

Literature: Luangsuphabool et al., 2016

69. Astrothelium neovariolosum Luangsuphabool, Aptroot \& Sangvichien, Lichenologist 48: 734 (2016)

Literature: Luangsuphabool et al., 2016

70. Astrothelium nitidiusculum (Nyl.) Aptroot \& Lücking, Lichenologist 48: 873 (2016)

Literature: Aptroot et al., 2007 as Trypethelium nitidiusculum; Aptroot and Lücking 2016

71. Astrothelium siamense Luangsuphabool, Aptroot \& Sangvichien, Lichenologist 48: 735 (2016)

Literature: Luangsuphabool et al., 2016 
72. Astrothelium subdiscretum (Nyl.) Aptroot \& Lücking, Lichenologist 48: 890 (2016)

Literature: Vongshewarat et al., 1999; Wolseley et al., 2002, both as Laurera subdiscreta and as Laurera phaeomelodes; Aptroot and Lücking 2016

73. Astrothelium subdissocians (Nyl. ex Vain.) Aptroot \& Lücking, Lichenologist 48: 891 (2016)

Literature: Vainio 1909, 1921 as Pseudopyrenula ochroleuca var. subdissocians; Aptroot and Lücking 2016

74. Astrothelium variolosum (Ach.) Müll. Arg., Flora, Regensburg 68(12): 255 (1885)

Literature: Wolseley et al., 2002 as Trypethelium variolosum; Aptroot et al., 2007; Aptroot and Lücking 2016

75. Aulaxina microphana (Vain.) R.Sant., Symb. Bot. Upsal. 12: 299 (1952)

Literature: Papong et al., 2007

76. Aulaxina quadrangula (Stirt.) R.Sant., J. Ecol. 40: 129 (1952)

Literature: Aptroot et al., 2007

77. Austrotrema terebrans (Nyl.) I. Medeiros, Lücking \& Lumbsch, Fieldiana 9: 17 (2017)

Literature: Medeiros et al., 2017; Schumm and Aptroot 2012, as Thelotrema bicinctulum

78. Bacidia heterochroa (Müll.Arg.) Zahlbr., Cat. Lich. Univ. 4: 204 (1926) Literature: Aptroot et al., 2007

79. Bacidia hostheleoides (Nyl.) Zahlbr., Cat. Lich. Univ. 4: 205 (1926) [1927] Literature: Schumm and Aptroot 2012

80. Bacidia manilensis var. siamensis Vain., Suom. Elian-ja Kasvit. Seuran Van. Julk. 1: 46 (1921)

Literature: Vainio 1921; Wolseley et al., 2002

81. Bacidia medialis (Tuck.) Zahlbr., Denkschr. Kaiserl. Akad. Wiss., Wien. Math.-Naturwiss. Kl. 83: 127 (1909)

Literature: Aptroot et al., 2007; Schumm and Aptroot 2012

82. Bacidia squamellosa (S.Ekman) Coppins \& Aptroot, Lichenologist 40: 367 (2008) Literature: Aptroot et al., 2007 as Bacidina squamellosa

83. Bacidia subincompta (Nyl.) Arnold, Flora 53: 472 (1870)

Literature: Paulson 1930 as Bacidia atrosanguinea; Wolseley et al., 2002 as Bacidia affinis

84. Bacidina pallidocarnea (Müll.Arg.) Vězda, Folia Geobot. Phytotax. 25: 432 (1991)

Literature: Papong et al., 2007

85. Bacidina penicillata Aptroot, M.Cáceres, Lücking \& Sparrius, in Aptroot et al., Fungal Diversity 24: 87 (2007)

Literature: Aptroot et al., 2007

86. Bacidiopsora orizabana (Vain.) Kalb, Biblioth. Lichenol. 88: 304 (2004) Literature: Aptroot et al., 2007 
87. Bacidiopsora squamulosula (Nyl.) Kalb, Lichenes Neotropici, Fascicle 10: 4 (1988) Literature: Aptroot et al., 2007

88. Bactrospora metabola (Nyl.) Egea \& Torrente, Mycotaxon 53: 58 (1995)

89. Bactrospora inspersa Aptroot, in Aptroot et al., Fungal Diversity 24: 89 (2007) Literature: Aptroot et al., 2007

90. Bactrospora myriadea (Fée) Egea \& Torrente, Lichenologist 25: 245 (1993) Literature: Kalb et al., 2012

91. Bactrospora perspiralis Sparrius, Saipunkaew \& Wolseley, Lichenologist 38: 29 (2006)

Literature: Sparrius et al., 2006

92. Bactrospora subdryina Sparrius, Saipunkaew \& Wolseley, Lichenologist 38: 28 (2006)

Literature: Sparrius et al., 2006

93. Badimia multiseptata Papong \& Lücking, Phytotaxa 18: 19 (2011)

Literature: Lumbsch et al., 2011

94. Bagliettoa baldensis (A.Massal.) Vězda, in Poelt \& Vězda, Biblioth. Lichenol. 16: 363 (1981)

Literature: Aptroot et al., 2007

95. Bathelium albidoporum (Makhija \& Patw.) Aptroot \& Lücking, Lichenologist 48: 897 (2016)

Literature: Aptroot and Lücking 2016

96. Bathelium madreporiforme (Eschw.) Trevis., Flora 44: 21 (1861)

Literature: Boonpragob et al., 1998 as Laurera madreporiformis; Wolseley et al., 2002; Aptroot et al., 2007; Aptroot and Lücking 2016

97. Bathelium nigroporum (Makhija \& Patw.) Aptroot \& Lücking, Lichenologist 48: 900 (2016)

Literature: Vongshewarat et al., 1999; Wolseley et al., 2002 as Trypethelium nigroporum

98. Blastenia testaceorufa (Vain.) Zahlbr., Cat. Lich. Univers. 7: 43 (1930)

Literature: Vainio 1909; Alava 1988; Wolseley et al., 2002 as Placodium testaceorufum

99. Bogoriella fumosula (Zahlbr.) Aptroot \& Lücking, Lichenologist 48: 910 (2016)

Literature: Aptroot et al., 2007 as Mycomicrothelia fumosula

100. Bogoriella hemisphaerica (Müll.Arg.) Aptroot \& Lücking, Lichenologist 48: 910 (2016)

Literature: Aptroot et al., 2007 as Mycomicrothelia hemisphaerica

101. Bogoriella miculiformis (Nyl. ex Müll.Arg.) Aptroot \& Lücking, Lichenologist 48: 912 (2016)

Literature: Aptroot et al., 2007 as Mycomicrothelia miculiformis

102. Bogoriella subfallens (Müll.Arg.) D Aptroot \& Lücking, Lichenologist 48: 915 (2016)

Literature: Aptroot et al., 2007 as Mycomicrothelia subfallens 
103. Brigantiaea leucoxantha (Spreng.) R.Sant. \& Hafellner, in Hafellner \& Bellemère, Nova Hedwigia 35: 246 (1982) [1981]

Literature: Boonpragob et al., 1998; Wolseley et al., 2002

104. Brigantiaea lobulatisidiata Aptroot, in Aptroot et al., Fungal Diversity 24: 90 (2007) Literature: Aptroot et al., 2007

105. Brigantiaea pulchra (Müll.Arg.) Hafellner, Nova Hedwigia 35: 707 (1983)

Literature: Wolseley and Aguirre-Hudson 1997b

106. Brigantiaea tricolor (Mont.) Trevis., Spighe Paglie: 9 (1853)

Literature: Wolseley and Aguirre-Hudson 1997a; Wolseley et al., 2002

107. Buellia aethalea (Ach.) Th.Fr., Lich. Scand. 1: 604 (1874)

Literature: Aptroot et al., 2007; Mongkolsuk and Meesim 2012

108. Buellia alboatrior (Nyl.) Zahlbr., Cat. Lich. Univ. 7: 441 (1931)

Literature: Van den Boom et al., 2014

109. Buellia almeriensis Llimona, Lichenes Selecti Exsiccati, Fascicle 48: 7 (1973)

Literature: Meesim and Mongkolsuk 2010b

110. Buellia amblyogona Müll.Arg., Bull. Herb. Boissier 3: 641 (1895)

Literature: Mongkolsuk and Meesim 2012

111. Buellia bahiana Malme, Ark. Bot. 21: 17 (1927)

Literature: Meesim and Mongkolsuk 2010a

112. Buellia blastenioides Vain., Hedwigia 46: 171 (1907)

Literature: Vainio 1909; Alava 1988; Wolseley et al., 2002

113. Buellia chloroleuca Körb., Parerga Lichenol.: 191 (1860)

Literature: Meesim and Mongkolsuk 2010b

114. Buellia circumpallida H. Magn., Ark. Bot. 3(10): 379 (1955)

Literature: Aptroot et al., 2007 as Endohyalina circumpallida

115. Buellia curatellae Malme, Ark. Bot. 21A(no. 14): 18 (1927)

Literature: Aptroot et al., 2007; Meesim and Mongkolsuk 2010b

116. Buellia demutans Zahlbr., Cat. Lich. Univers. 7: 348 (1931)

Literature: Aptroot et al., 2007 as Hafellia demutans

117. Buellia desertica (Marbach) Bungartz, Lichen Flora of the Greater Sonoran Desert Region (Tempe) 3: 138 (2007)

Literature: Meesim and Mongkolsuk 2010b

118. Buellia disciformis (Fr.) Mudd, Man. Brit. Lich.: 216 (1861)

Literature: Vainio 1921; Meesim and Mongkolsuk 2010b; Wolseley et al., 2002 as Hafellia disciformis

119. Buellia dispersa A.Massal., Sched. Crit. 8: 150 (1856)

Literature: Mongkolsuk and Meesim 2012

120. Buellia erubescens Arnold, Verh. Zool.-Bot. Ges. Wien 23: 493 (1874) [1873]

Literature: Meesim and Mongkolsuk 2010b; Mongkolsuk and Meesim 2012

121. Buellia glaucotheca Malme, Ark. Bot. 21: 8 (1927)

Literature: Meesim and Mongkolsuk 2010b

122. Buellia homophylia (C.Knight) Zahlbr., Cat. Lich. Univ. 7: 366 (1931)

Literature: Mongkolsuk and Meesim 2012 
123. Buellia insignis (Nägeli) Th.Fr., Nova Acta Soc. Regiar Sci. Upsal., 3: 327 (1861) [1860]

Literature: Meesim and Mongkolsuk 2010b

124. Buellia leptocline A.Massal., Geneac. Lich.: 20 (1854)

Literature: Meesim and Mongkolsuk 2010b

125. Buellia leptoclinoides (Nyl.) J. Steiner, Verh. zool.-bot. Ges. Wien 57: 357 (1907)

Literature: Meesim and Mongkolsuk 2010b

126. Buellia ocellata (Flörke ex Flot.) Körb., Syst. Lich. Germ.: 224 (1855)

Literature: Aptroot et al., 2007

127. Buellia pallidomarginata A.Nordin, Symb. Bot. Upsal. 33: 1-117 (2000)

Literature: Mongkolsuk and Meesim 2012; Van den Boom et al., 2014

128. Buellia parastata (Nyl.) Zahlbr., Cat. Lich. Univ. 7: 386 (1930)

129. Buellia pinicola S.R.Singh \& D.D.Awasthi, Biol. Mem. 6: 181 (1981) Literature: Meesim and Mongkolsuk 2010b

130. Buellia pleiophoroides var. meiospermoides Vain. ex Räsänen, J. Jap. Bot. 16: 141 (1940) Literature: Vainio 1909; Alava 1988; Wolseley and Aguirre-Hudson 1997b; Wolseley et al., 2002 as Buellia pleiophoroides

131. Buellia pleiotera Malme, Ark. Bot. 21A(14): 7 (1927)

Literature: Meesim and Mongkolsuk 2010b; Van den Boom et al., 2014

132. Buellia proximata H.Magn., Ark. F. Bot. 32A: 52 (1945)

Literature: Van den Boom et al., 2014

133. Buellia pruinosa Müll. Arg., Bull. Herb. Boissier 1: 51 (1893)

Literature: Van den Boom et al., 2014

134. Buellia rechingeri Zahlbr., in Rechinger, Denkschr. Kaiserl. Akad. Wiss. Wien, Math.-Naturwiss. Kl.: 274 (1907)

Literature: Aptroot et al., 2007 as Hafellia rechingeri

135. Buellia remensa (Stirt.) Imshaug, Farlowia 4: 502 (1955)

Literature: Meesim and Mongkolsuk 2010b as Buellia tincta

136. Buellia saxorum A.Massal., Ric. Auton. Lich. Crost.: 82 (1852)

Literature: Meesim and Mongkolsuk 2010b

137. Buellia schaereri De Not., G. Bot. Ital. 2: 199 (1846)

Literature: Meesim and Mongkolsuk 2010b

138. Buellia sequax (Nyl.) Zahlbr., Cat. Lich. Univ. 7: 410 (1931)

Literature: Meesim and Mongkolsuk 2010b as Buellia abstracta

139. Buellia stellulata (Taylor) Mudd, Man. Brit. Lich.: 216 (1861)

Literature: Meesim and Mongkolsuk 2010b

140. Buellia stigmea Tuck., Syn. N. Amer. Lich. 2: 90 (1888)

Literature: Meesim and Mongkolsuk 2010a, 2010b

141. Buellia stillingiana J.Steiner, Oesterr. Bot. Z. 18: 144 (1919)

Literature: Meesim and Mongkolsuk 2010b

142. Buellia subnexa Vain., Bot. Tidsskr. 29: 115 (1909)

Literature: Vainio 1907, 1909; Alava 1988; Wolseley et al., 2002 as Buellia subdives 
143. Buellia subdisciformis (Leight.) Jatta, Syll. Lich. Ital.: 392 (1900)

Literature: Aptroot et al., 2007; Meesim and Mongkolsuk 2010b

144. Buellia subsororioides S.R.Singh \& D.D.Awasthi, Biol. Mem. 6: 191 (1981)

Literature: Meesim and Mongkolsuk 2010b

145. Buellia substigmea S.R.Singh \& D.D.Awasthi, Biol. Mem. 6: 191 (1981)

Literature: Meesim and Mongkolsuk 2010b

146. Buellia triseptata A.Nordin, Bryologist 102: 260 (1999)

Literature: Meesim and Mongkolsuk 2010b

147. Bulbothrix bulbochaeta (Hale) Hale, Phytologia 28: 480 (1974)

Literature: Saipunkaew et al., 2007; Louwhoff et al., 2012

148. Bulbothrix goebelii (Zenker) Hale, Smithsonian Contr. Bot. 32: 14 (1976)

Literature: Vainio 1909; Hale 1976a as Parmelia addenda; Wolseley and Aguirre-Hudson 1997a; Wolseley et al., 2002; Louwhoff et al., 2012

149. Bulbothrix hypocraea (Vain.) Hale, Phytologia 28: 480 (1974)

Literature: Pooprang et al., 1999; Wolseley et al., 2002; Buaruang et al., 2009; Louwhoff et al., 2012

150. Bulbothrix isidiza (Nyl.) Hale, Phytologia 28: 480 (1974)

Literature: Wolseley and Aguirre-Hudson 1995, 1997a, 1997b; Moon et al., 2000a; Wolseley et al., 2002; Buaruang et al., 2009; Louwhoff et al., 2012; Schumm and Aptroot 2012

151. Bulbothrix laevigatula (Nyl.) Hale, Phytologia 28: 480 (1974) Literature: Vainio 1921 as Parmelia hookeri; Wolseley et al., 2002

152. Bulbothrix meizospora (Nyl.) Hale, Phytologia 28: 480 (1974) Literature: Boonpragob et al., 1998; Pooprang et al., 1999; Wolseley et al., 2002; Louwhoff et al., 2012; Schumm and Aptroot 2012

153. Bulbothrix papyrina (Fée) Hale, Phytologia 28: 480 (1974) Literature: Vainio 1909 as Parmelia addenda

154. Bulbothrix pigmentacea (Hale) Hale, Phytologia 28: 480 (1974)

Literature: Wolseley and Aguirre-Hudson 1995, 1997a, 1997b; Wolseley et al., 2002; Louwhoff et al., 2012

155. Bulbothrix queenslandica (Elix \& G.N.Stevens) Elix, Mycotaxon 47: 126 (1993)

Literature: Pooprang et al., 1999; Wolseley et al., 2002

156. Bulbothrix sensibilis (J.Steiner \& Zahlbr.) Hale, Phytologia 28: 481 (1974)

Literature: Pooprang et al., 1999; Wolseley et al., 2002

157. Bulbothrix setschwanensis (Zahlbr.) Hale, Phytologia 28: 481 (1974)

Literature: Wolseley and Aguirre-Hudson 1997a; Wolseley et al., 2002; Louwhoff et al., 2012

158. Bulbothrix tabacina (Mont. \& Bosch) Hale, Phytologia 28: 481 (1974)

Literature: Satô 1962 as Parmelia meizosporoides; Buaruang et al., 2009; Louwhoff et al., 2012

159. Bulbothrix ventricosa (Hale \& Kurok.) Hale, Phytologia 28: 481 (1974)

Literature: Papong et al., 2007 
160. Byssolecania fumosonigricans (Müll.Arg.) R.Sant., Symb. Bot. Upsal. 12: 553 (1952)

Literature: Aptroot et al., 2007

161. Byssolecania hymenocarpa (Vain.) Kalb, Vězda \& Lücking, in Lücking \& Kalb, Bot. Jahrb. Syst.122: 21 (2000)

Literature: Papong et al., 2007

162. Byssoloma annuum (Vain.) Thor, Lücking \& Tat.Matsumoto, Symb. Bot. Upsal. 32: 29

Literature: Papong et al., 2007

163. Byssoloma chlorinum (Vain.) Zahlbr., Cat. Lich. Univ. 8: 233 (1932)

Literature: Boonpragob et al., 1998; Wolseley et al., 2002

164. Byssoloma fadenii Vězda, Folia Geobot. Phytotax. 10: 425 (1975)

Literature: Papong et al., 2007

165. Byssoloma gahavisukanum Sérus., in Aptroot et al., Biblioth. Lichenol. 64: 39 (1997) Literature: Papong et al., 2007

166. Byssoloma guttiferae (Bat. \& Peres) Lücking \& Sérus., in Lücking et al., Lichenologist 30: 138 (1998)

Literature: Papong et al., 2007

167. Byssoloma leucoblepharum (Nyl.) Vain., Dansk Bot. Ark. 4: 23 (1926)

Literature: Wolseley and Aguirre-Hudson 1997a; Boonpragob et al., 1998;

Wolseley et al., 2002

168. Byssoloma subdiscordans (Nyl.) P.James, Lichenologist 5: 126 (1971)

Literature: Aptroot et al., 2007; Papong et al., 2007

169. Byssoloma tricholomum (Mont.) Zahlbr., Cat. Lich. Univ. 2: 569 (1923) [1924]

Literature: Boonpragob et al., 1998; Wolseley et al., 2002

170. Calenia aspidota (Vain.) Vězda, Folia Geobot. Phytotax. 19: 195 (1984)

Literature: Aptroot et al., 2007

171. Calenia depressa Müll.Arg., Lichenes Epiphylli Novi: 4 (1890)

Literature: Papong et al., 2007

172. Calenia graphidea Vain., Ann. Acad. Sci. Fnn., Ser. A, 15: 163 (1921)

Literature: Boonpragob et al., 1998; Wolseley et al., 2002

173. Calenia leptocarpa Vain., Ann. Acad. Sci. Fenn., Ser. A, 15: 163 (1921)

Literature: Papong et al., 2007

174. Calenia phyllogena (Müll.Arg.) R.Sant., Symb. Bot. Upsal. 12: 349 (1952)

Literature: Boonpragob et al., 1998; Wolseley et al., 2002

175. Calenia thelotremella Vain., Ann. Acad. Sci. Fenn., Ser. A, 15: 160 (1921)

Literature: Papong et al., 2007

176. Calicium adaequatum Nyl., Flora 52: 409 (1869)

Literature: Aptroot et al., 2007

177. Calicium diploellum Nyl., Flora 51: 161 (1868)

Literature: Aptroot et al., 2007

178. Calicium hyperelloides Nyl., Syn. Meth. Lich. 1: 153 (1860)

Literature: Aptroot et al., 2007 
179. Calopadia fusca (Müll.Arg.) Vězda, Folia Geobot. Phytotax. 21: 215 (1986) Literature: Boonpragob et al., 1998; Wolseley et al., 2002

180. Calopadia puiggarii (Müll.Arg.) Vězda, Folia Geobot. Phytotax. 21:215 (1986) Literature: Aptroot et al., 2007; Papong et al., 2007

181. Calopadia subcoerulescens (Zahlbr.) Vězda, Lichenes Selecti Exsiccati 88: 3 (1988)

Literature: Aptroot et al., 2007

182. Caloplaca cinnabarina (Ach.) Zahlbr., in Engler \& Prantl, Nat. Pflanzenfam. Nachtr. [Engler \& Prantl] 1: 228 (1908)

Literature: Paulson 1930; Wolseley et al., 2002 as Caloplaca aequata; Aptroot et al., 2007

183. Caloplaca flavorubescens (Huds.) J.R.Laundon, Lichenologist 8: 147 (1976) Literature: Paulson 1930 as Caloplaca aurantiaca; Wolseley et al., 2002

184. Caloplaca flavovirescens (Wulfen) Dalla Torre \& Sarnth., Fl. Tirol: 180 (1902) Literature: Aptroot et al., 2007

185. Caloplaca holocarpa (Hoffm.) A.E.Wade, Lichenologist 3: 11 (1965) Literature: Paulson 1930 as Caloplaca pyracea; Wolseley et al., 2002

186. Caloplaca inconnexa (Nyl.) Zahlbr., Cat. Lich. Univ. 7: 145 (1930) [1931] Literature: Aptroot et al., 2007

187. Caloplaca pulicarioides Aptroot, in Aptroot \& Seaward, Trop. Bryol. 17: 69 (1999)

Literature: Aptroot et al., 2007

188. Candelaria concolor (Dicks.) Arnold, Flora 62: 364 (1879)

Literature: Wolseley and Aguirre-Hudson 1995

189. Candelariella reflexa (Nyl.) Lettau, Hedwigia 52: 196 (1912)

Literature: Aptroot et al., 2007

190. Canoparmelia concrescens (Vain.) Elix \& Hale, in Elix et al., Mycotaxon 27: 278 (1986)

Literature: Moon et al., 2000a; Wolseley et al., 2002; Louwhoff et al., 2012

191. Canoparmelia ecaperata (Müll.Arg.) Elix \& Hale, in Elix et al., Mycotaxon 27: 278 (1986)

Literature: Vainio 1921; Alava 1988 as Parmelia malaccensis var. laeteflavens; Hale 1976c as Pseudoparmelia ecaperata; Wolseley and Aguirre-Hudson 1995, 1997a, 1997b; Boonpragob et al., 1998; Moon et al., 2000a; Wolseley et al., 2002; Louwhoff et al., 2012

192. Canoparmelia owariensis (Asahina) Elix, Mycotaxon 47: 127 (1993)

Literature: Hale 1976c as Pseudoparmelia owariensis; Moon et al., 2000a; Wolseley et al., 2002; Buaruang et al., 2009; Louwhoff et al., 2012

193. Canoparmelia pustulescens (Kurok.) Elix, Mycotaxon 47: 127 (1993)

Literature: Moon et al., 2000a; Wolseley et al., 2002

194. Canoparmelia rodriguesiana (Hue) Elix, Mycotaxon 70: 108 (1999) Literature: Moon et al., 2000a; Wolseley et al., 2002 as Paraparmelia rodriguesiana 
195. Canoparmelia texana (Tuck.) Elix \& Hale, in Elix et al., Mycotaxon 27: 279 (1986)

Literature: Hale 1976c as Pseudoparmelia texana; Moon et al., 2000a; Wolseley et al., 2002; Louwhoff et al., 2012

196. Caprettia amazonensis Bat. \& H.Maia, Atas Inst. Micol. Univ. Recife 2: 378 (1965)

Literature: Papong et al., 2007

197. Caprettia nyssaegenoides Sérus. \& Lücking, Biblioth. Lichenol. 86: 169 (2003) Literature: Schumm and Aptroot 2012

198. Carbacanthographis amicta (Nyl.) Staiger \& Kalb, in Staiger, Biblioth. Lichenol. 85: 103 (2002)

Literature: Aptroot et al., 2007

199. Carbacanthographis candidata (Nyl.) Staiger \& Kalb, in Staiger, Biblioth. Lichenol. 85: 104 (2002)

Literature: Aptroot et al., 2007; Poengsungnoen et al., 2010

200. Carbacanthographis marcescens (Fée) Staiger \& Kalb, in Staiger, Biblioth. Lichenol. 85: 109 (2002)

Literature: Aptroot et al., 2007; Wolseley et al., 2002 as Graphina intricata; Poengsungnoen et al., 2010 as Graphina marcescens

201. Catillaria testaceolivens (Vain.) Zahlbr., Cat. Lich. Univ. 4: 79 (1926) [1927]

Literature: Vainio 1909 as Lecidea testaceolivens; Wolseley et al., 2002

202. Catillaria unicolor (Vain.) Zahlbr., Cat. Lich. Univ. 4: 83 (1926) [1927]

Literature: Vainio 1909; Alava 1988 as Lecidea unicolor; Wolseley et al., 2002

203. Celothelium aciculiferum (Nyl.) Vain., Ann. Acad. Sci. Fenn., Ser. A. 6: 209 (1915) Literature: Aptroot et al., 2007

204. Cetrelia braunsiana (Müll.Arg.) W.L.Culb. \& C.F.Culb., Contr. U.S. Natl. Herb. 34: 493 (1968)

Literature: Satô 1962 as Cetraria collata f. isidiata; Wolseley and Aguirre-Hudson 1997a; Wolseley et al., 2002; Louwhoff et al., 2012

205. Cetrelia japonica (Zahlbr.) W.L.Culb. \& C.F.Culb., Contr. U.S. Natl. Herb. 34: 511 (1968)

Literature: Wolseley and Aguirre-Hudson 1997a; Boonpragob et al., 1998; Wolseley et al., 2002; Louwhoff et al., 2012

206. Cetrelia nuda (Hue) W.L.Culb. \& C.F.Culb., Contr. U.S. Natl. Herb. 34: 513 (1968)

Literature: Wolseley and Aguirre-Hudson 1997a; Wolseley et al., 2002; Louwhoff et al., 2012

207. Cetrelia olivetorum (Nyl.) W.L.Culb. \& C.F.Culb., Contr. U.S. Natl. Herb. 34: 515 (1968)

Literature: Wolseley and Aguirre-Hudson 1997a; Wolseley et al., 2002; Louwhoff et al., 2012

208. Chaenotheca trichialis (Ach.) Hellb., K. Svenska Vet. Akad. Handl. 9: 82 (1870) Literature: Boonpragob et al., 1998; Wolseley et al., 2002 
209. Chapsa alborosella (Nyl.) Frisch, Biblioth. Lichenol. $92: 90$ (2006)

Literature: Boonpragob et al., 1998; Wolseley et al., 2002 as Thelotrema albosorellum

210. Chapsa discoides (Stirt.) Lücking, in Sipman et al., Phytotaxa 55: 35 (2012) Literature: Papong et al., 2014

211. Chapsa imperfecta (Hale) Rivas Plata \& Mangold, in Rivas Plata et al., Lichenologist 42: 183 (2010)

Literature: Wolseley et al., 2002 as Thelotrema imperfectum

212. Chapsa indica A.Massal., Atti Ist. Veneto Sci. Lett. Arti 5: 257 (1860) [1859-1860] Literature: Wolseley et al., 2002 as Thelotrema pycnophragmium; Schumm and Aptroot 2012; Parnmen, et al., 2012; Kraichak, et al., 2013; Lumbsch et al., 2014; Poengsungnoen et al., 2014a

213. Chapsa laemensis (Homchant. \& Coppins) Lumbsch \& Papong, in Papong et al., Lichenologist 42: 136 (2010)

Literature: Yoshimura 1978; Homchantara and Coppins 2002; Wolseley et al., 2002 as Thelotrema laemense; Papong et al., 2010

214. Chapsa leprocarpa (Nyl.) Frisch, Biblioth. Lichenol. 92: 108 (2006)

Literature: Boonpragob et al., 1998 as Thelotrema colobicum Wolseley et al., 2002 as Thelotrema colobicum and Thelotrema leprocarpum; Parnmen, et al., 2012; Kraichak, et al., 2013; Lumbsch et al., 2014; Poengsungnoen et al., 2014a

215. Chapsa leprocarpoides (Hale) M. Cáceres \& Lücking, in Cáceres, Libri Botanici 22: 52 (2007)

Literature: Boonpragob et al., 1998; Wolseley et al., 2002 as Thelotrema leprocarpoides

216. Chapsa patens (Nyl.) Frisch, Biblioth. Lichenol. 92: 111 (2006)

Literature: Wolseley and Aguirre-Hudson 1997b; Wolseley et al., 2002 as Thelotrema patens; Parnmen, et al., 2012; Kraichak, et al., 2013; Lumbsch et al., 2014; Poengsungnoen et al., 2014a

217. Chiodecton congestulum Nyl., Bull. Soc. Linn. Normandie 2: 106 (1868)

Literature: Wolseley et al., 2002

218. Chiodecton leptosporum Müll.Arg., Flora 65: 332 (1882)

Literature: Aptroot et al., 2007

219. Chiodecton mucorinum Zahlbr. in Handel-Mazzetti, Symb. Sin. 3: 63 (1930)

Literature: Aptroot et al., 2007; Schumm and Aptroot 2012

220. Chiodecton subsphaerale Nyl., J. Linn. Soc., Bot. 20: 59 (1883)

Literature: Vainio 1909; Wolseley et al., 2002

221. Chroodiscus australiensis Vězda \& Lumbsch, in Lumbsch \& Vězda, Nova Hedwigia 50: 246 (1990)

Literature: Papong et al., 2007

222. Chroodiscus defectus Papong \& Lücking, in Papong et al. Bryologist 112: 155 (2009)

Literature: Papong et al., 2009b; Parnmen et al., 2012; Lumbsch et al., 2014; Poengsungnoen et al., 2014a 
223. Chroodiscus homchantarae Papong \& Lücking, in Papong et al. Bryologist 112: 155 (2009)

Literature: Boonpragob et al., 1998; Wolseley et al., 2002 as Chroodiscus mirificus; Papong et al., 2009b

224. Chroodiscus khaolungensis Papong \& Lücking, in Papong et al. Bryologist 112: 156 (2009)

Literature: Papong et al., 2009b; Parnmen et al., 2012; Lumbsch et al., 2014

225. Chroodiscus khaosokensis Papong \& Lücking, in Papong et al. Bryologist 112: 156 (2009)

Literature: Papong et al., 2009b; Poengsungnoen et al., 2014a

226. Chroodiscus parvisporus Kalb \& Lücking, in Lücking \& Grube, Stapfia 80: 271 (2002)

Literature: Papong et al., 2007; Parnmen et al., 2012

227. Chroodiscus verrucosus R.Sant., Lücking \& Vězda, in Santesson \& Lücking, Nordic J. Bot. 19: 727 (1999)

Literature: Parnmen et al., 2012; Lumbsch et al., 2014; Poengsungnoen et al., $2014 \mathrm{a}$

228. Chrysothrix xanthina (Vain.) Kalb, Biblioth. Lichenol. 78: 144 (2001)

Literature: Vainio 1909 as Lepraria xanthina; Wolseley and Aguirre-Hudson 1997a, 1997b; Wolseley et al., 2002 as Chrysothrix candelaris; Kalb et al., 2009a

229. Cladia aggregata (Sw.) Nyl., Recogn. Ram. 69: 167 (1870)

Literature: Yoshimura 1978; Wolseley and Aguirre-Hudson 1995; Wolseley et al., 2002; Parnmen et al., 2008

230. Cladonia calyciformis Nuno, J. Jap. Bot. 47: 161 (1972)

Literature: Yoshimura 1978; Wolseley et al., 2002

231. Cladonia ciliata var. tenuis (Flörke) Ahti, Best. Europ. Flecht., Ergdnz. 1: 68 (1977)

Literature: Yoshimura 1978 as Cladonia tenuis; Wolseley et al., 2002

232. Cladonia corymbescens Nyl., in Leighton, Ann. Mag. Nat. Hist. 18: 407 (1866) Literature: Parnmen et al., 2008

233. Cladonia didyma var. vulcanica (Zoll. \& Moritzi) Vain., Acta Soc. Fauna Fl. Fenn. 4: 145 (1887)

Literature: Yoshimura 1978 as Cladonia vulcanica; Wolseley et al., 2002

234. Cladonia fruticulosa Kremp., Verh. Zool.-Bot. Ges. Wien 30: 331 (1880)

Literature: Parnmen et al., 2008

235. Cladonia furcata (Huds.) Schrad., Spicil. Fl. Germ. 1: 107 (1794)

Literature: Boonpragob et al., 1998; Wolseley et al., 2002; Parnmen et al., 2008

236. Cladonia gymnopoda Vain., Acta Soc. Fauna Fl. Fenn. 10: 172 (1894)

Literature: Yoshimura 1978; Wolseley et al., 2002

237. Cladonia homchantarae Ahti \& Parnmen, in Ahti et al., Sauteria 15: 15 (2008) Literature: Ahti et al., 2008; Parnmen et al., 2008 
238. Cladonia macilenta Hoffm., Deutschl. Fl., Theil 2 (Hoffm.) 2: 126 (1796) [1795]

Literature: Parnmen et al., 2008

239. Cladonia modesta Ahti \& Krog, Ann. Bot. Fenn. 24: 88 (1987)

Literature: Boonpragob et al., 1998; Wolseley et al., 2002

240. Cladonia mongkolsukii Parnmen \& Ahti., Phytotaxa 18: 41 (2011)

Literature: Lumbsch et al., 2011

241. Cladonia mauritiana Ahti \& J.C. David, in David \& Hawksworth, Biblioth. Lichenol. 57: 94 (1995)

Literature: Parnmen et al., 2008

242. Cladonia ochrochlora Flörke, De Cladoniis, Difficillimo lichenum genere, Commentatio nova: 75 (1828)

Literature: Parnmen et al., 2008

243. Cladonia ramulosa (With.) J.R.Laundon, Lichenologist 16: 225 (1984)

Literature: Vainio 1921; Yoshimura 1978 as Cladonia pityrea; Wolseley et al., 2002

244. Cladonia rangiferina (L.) Weber ex F.H.Wigg., in Wiggers, Prim. Fl. Holsat.: 90 (1780)

Literature: Yoshimura 1978; Wolseley and Aguirre-Hudson 1995; Wolseley et al., 2002

245. Cladonia rappii A. Evans, Trans. Ky Acad. Sci. 38: 297 (1952)

Literature: Parnmen et al., 2008

246. Cladonia recticaulis Ahti \& Parnmen, in Ahti et al., Sauteria 15: 16 (2008)

Literature: Ahti et al., 2008; Parnmen et al., 2008

247. Cladonia rudis Ahti \& Parnmen, in Ahti et al., Sauteria 15: 17 (2008)

Literature: Ahti et al., 2008; Parnmen et al., 2008

248. Cladonia scabriuscula (Delise) Leight., Flora 58: 447 (1875)

Literature: Parnmen et al., 2008

249. Cladonia siamea Abbayes, Kew Bull. 2: 262 (1956)

Literature: Wolseley and Aguirre-Hudson 1995; Yoshimura 1978; Wolseley et al., 2002

250. Cladonia singhii Ahti \& P.K.Dixit, in Ahti et al., Lichenologist 34: 306 (2002) Literature: Parnmen et al., 2008

251. Cladonia squamosa (Scop.) Hoffm., Deutschl. Fl., Theil 2 (Hoffm.) 2: 125 (1796) [1795]

Literature: Boonpragob et al., 1998; Wolseley et al., 2002

252. Cladonia subcervicornis (Vain.) Kernst., Éur. Clad.: 15 (1900)

Literature: Yoshimura 1978 as Cladonia calycantha; Wolseley et al., 2002;

Parnmen et al., 2008 as Cladonia rappii

253. Cladonia subpityrea Sandst., in Keissler, Ann. Naturhist. Mus. Wien 42: 62 (1928)

Literature: Yoshimura 1978; Wolseley et al., 2002 
254. Cladonia transindica Ahti, Ann. Bot. Soc. Zool.-Bot. Fenn. "Vanamo" 32: 72 (1961)

Literature: Wolseley and Aguirre-Hudson 1995; Wolseley et al., 2002

255. Clandestinotrema clandestinum (Ach.) Rivas Plata, Lücking \& Lumbsch, Fungal Diversity 52: 116 (2012)

Literature: Boonpragob et al., 1998 as Myriotrema clandestinum

256. Coccocarpia adnata Arv., Opera Bot. 67: 42 (1983)

Literature: Rangsiruji et al., 2016

257. Coccocarpia dissecta Swinscow \& Krog, Norweg. J. Bot. 23: 254 (1976)

Literature: Mongkolsuk et al., 1996; Wolseley et al., 2002; Rangsiruji et al., 2016

258. Coccocarpia erythroxyli (Spreng.) Swinscow \& Krog, Norweg. J. Bot. 23: 254 (1976)

Literature: Satô 1962 as Coccocarpia pellita var. parmelioides; Paulson 1930;

Vainio 1921 as Coccocarpia molybdaea; Boonpragob et al., 1998; Wolseley et al., 2002; Schumm and Aptroot 2012; Rangsiruji et al., 2016

259. Coccocarpia glaucina Kremp., Nuovo Giorn. Bot. Ital. 7: 14 (1875)

Literature: Boonpragob et al., 1998; Wolseley et al., 2002; Schumm and Aptroot 2012

260. Coccocarpia palmicola (Spreng.) Arv. \& D.J.Galloway, Bot. Not. 132: 242 (1979)

Literature: Wolseley and Aguirre-Hudson 1997a, 1997b; Boonpragob et al., 1998; Wolseley et al., 2002; Rangsiruji et al., 2016

261. Coccocarpia pellita (Ach.) Müll.Arg., Flora 65: 320 (1882)

Literature: Boonpragob et al., 1998; Wolseley et al., 2002; Rangsiruji et al., 2016

262. Coccocarpia smaragdina Pers., in Gaudichaud-Beaupré in Freycinet, Voy.

Uranie, Bot. 5: 206 (1827)

Literature: Paulson 1930

263. Coccotrema porinopsis (Nyl.) Imshaug ex Yoshim., Misc. Bryol. Lichenol. 6: 135 (1974)

Literature: Aptroot et al., 2007

264. Coenogonium barbatum Lücking, Aptroot \& Umaña, Fungal Diversity 23: 273 (2006)

Literature: Kalb et al., 2016a

265. Coenogonium convexum J. Kalb \& K. Kalb, in Kalb, Boonpragob \& Kalb, Phytotaxa 283: 108 (2016)

Literature: Kalb et al., 2016a

266. Coenogonium coronatum G.Neuwirth \& E.Stocker-Wörgötter, Bryologist 117: $161(2014)$

Literature: Neuwirth et al. 2014; Kalb et al., 2016a

267. Coenogonium dilucidum (Kremp.) Kalb \& Lücking, in Lücking \& Kalb, Bot. Jahrb. Syst. 122: 32 (2000)

Literature: Aptroot et al., 2007; Papong et al., 2007, Neuwirth et al. 2014; Kalb et al., 2016a 
268. Coenogonium disciforme Papong, Boonpragob \& Lücking, Lichenologist 39: 47 (2007)

Literature: Papong et al., 2007, Neuwirth et al. 2014

269. Coenogonium epiphyllum Vain., Ann. Acad. Sci. Fenn., Ser. A, 15: 156 (1921) Literature: Kalb et al., 2016a

270. Coenogonium flavicans (Vězda \& Farkas) Kalb \& Lücking, in Lücking \& Kalb, Bot. Jahrb. Syst. 122: 32 (2000)

Literature: Papong et al., 2007, Neuwirth et al. 2014

271. Coenogonium frederici (Kalb) Kalb \& Lücking, Fungal Diversity 23: 297 (2006).

Literature: Kalb et al., 2016a

272. Coenogonium geralense (P. Henn.) Lücking, Flora neotrop. Monogr. 103: 579 (2008).

Literature: Kalb et al., 2016a

273. Coenogonium interplexum Nyl., Ann. Sci. Nat., Bot. 16: 92 (1862)

Literature: Papong et al., 2007, Neuwirth et al., 2014; Kalb et al., 2016a

274. Coenogonium isidiiferum (Lücking) Lücking, Lichenologist 33: 201 (2001)

Literature: Neuwirth et al. 2014; Kalb et al., 2016a

275. Coenogonium leprieurii (Mont.) Nyl., Ann. Sci. Nat. Bot., Ser. 4, 16: 89 (1862) Literature: Kalb et al., 2016a

276. Coenogonium luteum (Dicks.) Kalb \& Lücking, Bot. Jahrb. Syst. 122: 32 (2000)

Literature: Vainio 1909 as Gyalecta lutea; Neuwirth et al., 2014; Kalb et al., $2016 \mathrm{a}$

277. Coenogonium nepalense (G.Thor \& Vězda) Lücking, Aptroot \& Sipman, in Rivas Plata, et al., Fungal Diversity 23: 297 (2006)

Literature: Sriprang and Mongkolsuk 2010 as Dimerella nepalensis; Neuwirth et al. 2014; Kalb et al., 2016a

278. Coenogonium pineti (Ach.) Lücking \& Lumbsch, in Lücking et al., Mycologia 96: 290 (2004)

Literature: Kalb et al., 2012; Kalb et al., 2016a

279. Coenogonium queenslandicum (Kalb \& Vězda) Lücking, Lücking et al., Lichenologist 33: 201 (2001)

Literature: Kalb et al., 2016a

280. Coenogonium subborinquense J. Kalb \& K. Kalb, in Kalb, Boonpragob \& Kalb, Phytotaxa 283: 118 (2016)

Literature: Kalb et al., 2016a

281. Coenogonium subluteum (Rehm) Kalb \& Lücking, in Lücking \& Kalb, Bot. Jahrb. Syst. 122: 34 (2000)

Literature: Aptroot et al., 2007, Neuwirth et al. 2014; Kalb et al., 2016a

282. Coenogonium verrucimarginatum J. Kalb \& K. Kalb, in Kalb, Boonpragob \& Kalb, Phytotaxa 283: 120 (2016)

Literature: Kalb et al., 2016a 
283. Collema coilocarpum (Müll.Arg.) Zahlbr., Cat. Lich. Univ. 3: 34 (1924) [1925] Literature: Wolseley and Aguirre-Hudson 1997a, 1997b; Boonpragob et al., 1998; Wolseley et al., 2002

284. Collema furfuraceum (Arn.) Du Rietz, Ark. Bot. 22A: 5 (1929)

Literature: Aptroot et al., 2007

285. Collema leptaleum Tuck., Proc. Amer. Acad. Arts 6: 263 (1866) [1864]

Literature: Aptroot et al., 2007

286. Collema leptaleum var. biliosum (Mont.) Degel., Symb. Bot. Upsal. 20: 105 (1974) Literature: Aptroot et al., 2007

287. Collema pulchellum var. subnigrescens (Müll.Arg.) Degel., Symb. Bot. Upsal. 20: 173 (1974)

Literature: Aptroot et al., 2007

288. Collema rugosum Kremp., in Fenzl, Reise Novara 1: 128 (1870)

Literature: Aptroot et al., 2007

289. Compositrema thailandicum Rivas Plata, Papong \& Lumbsch, Taxon 61: 1174 (2012)

Literature: Rivas Plata et al., 2012a; Lumbsch et al., 2014; Poengsungnoen et al., 2014a; Kraichak et al., 2014; Lücking et al., 2015

290. Constrictolumina cinchonae (Ach.) Lücking, M. P. Nelsen \& Aptroot, in Lücking et al., Lichenologist 48: 756. 2016.

Literature: Vainio 1909 as Didymella cinchonae; Aptroot et al., 2007 as Arthopyrenia cinchonae

291. Cratiria amphorea (Eckfeldt) Marbach, Biblioth. Lichenol. 74: 172 (2000) Literature: Aptroot et al., 2007

292. Cratiria dissimilis (Nyl.) Mabach, Biblioth. Lichenol. 74: 177 (2000) Literature: Schumm and Aptroot 2012

293. Cratiria lauricassiae (Fée) Marbach, Biblioth. Lichenol. 74: 160 (2000) Literature: Aptroot et al., 2007; Van den Boom et al., 2014

294. Cratiria melanochlora (Kremp.) Marbach, Biblioth. Lichenol. 74: 183 (2000) Literature: Aptroot et al., 2007; Mongkolsuk and Meesim 2012

295. Cratiria obscurior (Stirt.) Marbach \& Kalb, in Marbach, Biblioth. Lichenol. 74: 186 (2000)

Literature: Aptroot et al., 2007

296. Cratiria rutilans Marbach, Biblioth. Lichenol. 74: 193 (2000)

Literature: Aptroot et al., 2007

297. Cratiria rutilantoides Marbach, Biblioth. Lichenol. 74: 196 (2000) Literature: Aptroot et al., 2007

298. Creographa subbrasiliensis Sutjaritt. \& Kalb, Phytotaxa 189: 313 (2014) Literature: Sutjaritturakan and Kalb 2014

299. Cresponea chloroconia (Tuck.) Egea \& Torrente, Mycotaxon 48: 310 (1993) Literature: Vainio 1909 as Lecanactis deminuens; Wolseley et al., 2002

300. Cresponea leprieurii (Mont.) Egea \& Torrente, Mycotaxon 48: 317 (1993) Literature: Aptroot et al., 2007 
301. Cresponea proximata (Nyl.) Egea \& Torrente, Mycotaxon 48: 328 (1993)

Literature: Wolseley et al., 2002; Aptroot et al., 2007; Schumm and Aptroot 2012

302. Crocodia aurata (Ach.) Link, Handb. Erkennung Vork. Gewächs. 3: 177 (1833)

Literature: Wolseley and Aguirre-Hudson 1995; 1997a; Wolseley et al., 2002 as Pseudocyphellaria aurata

303. Crocodia clathrata (De Not.) Trevis., Lichenoth. Venet.: no. 75 (1869)

Literature: Aptroot et al., 2007 as Pseudocyphellaria clathrata

304. Crocynia gossypina (Sw.) A.Massal., Atti Ist. Veneto Sci. Lett. Arti 5: 252 (1860) [1859-1860]

Literature: Aptroot et al., 2007

305. Crocynia pyxinoides Nyl., Sert. Lich. Trop. Labuan Singapore: 37 (1891)

Literature: Wolseley and Aguirre-Hudson 1995; 1997a; 1997b; Boonpragob et al., 1998

306. Cruentotrema cruentatum (Mont.) Rivas Plata, Lumbsch \& Lücking, Fungal Diversity 52: 119 (2012)

Literature: Yoshimura 1978 as Thelotrema rhododiscum; Homchantara and Coppins 2002; Wolseley et al., 2002; Papong et al., 2010 as Ocellularia cruentata; Sutjaritturakan and Kalb 2015

307. Cruentotrema kurandense (Mangold) Rivas Plata, Lumbsch \& Lücking, in Rivas Plata, Lücking \& Lumbsch, Fungal Diversity52: 119 (2012)

Literature: Kalb et al., 2016b

308. Cruentotrema lirelliforme J. Kalb, Polyiam \& K. Kalb, in Kalb, Polyiam, Rivas Plata, Bawingan, Kalb \& Lücking, Phytotaxa 268: 115 (2016)

Literature: Kalb et al., 2016b

309. Cruentotrema thailandicum Rivas Plata, Papong \& Lumbsch, in Rivas Plata et al., Fungal Diversity 52: 119 (2012)

Literature: Rivas Plata et al., 2012b; Lumbsch et al., 2014

310. Crustospathula khaoyaiana Kalb \& Mongkolsuk, in Kalb et al., Phytotaxa 42: 35-47 (2012)

Literature: Kalb et al., 2012

311. Cryptolechia subincolorella (Nyl.) D.Hawksw. \& Dibben, Lichenologist 14: 100 (1982)

Literature: Aptroot et al., 2007

312. Cryptothecia candida (Kremp.) R.Sant., Symb. Bot. Upsal.12: 65 (1952)

Literature: Boonpragob et al., 1998; Wolseley et al., 2002

313. Cryptothecia eungellae G.Thor, Symb. Bot. Upsal. 32: 280 (1997)

Literature: Kalb et al., 2012

314. Cryptothecia obtecta Makhija \& Patw., Biovigyanam 13: 46 (1987)

Literature: Wolseley et al., 2002

315. Cryptothecia philippinum (Vain.) G.Thor, Symb. Bot. Upsal. 32: 283 (1997) Literature: Aptroot et al., 2007 
316. Cryptothecia punctosorediata Sparrius, in Sparrius \& Saipunkaew, Lichenologist 37: 507 (2005)

Literature: Sparrius and Saipunkaew 2005

317. Cryptothecia scripta G.Thor, Symb. Bot. Upsal.32: 285 (1997)

Literature: Aptroot et al., 2007

318. Cryptothelium sepultum (Mont.) A.Massal., Atti Ist. Veneto Sci. Lett. Arti 5: 335 (1860) [1859-1860]

Literature: Aptroot et al., 2007

319. Culbersonia nubila (Moberg) Essl., Lichen Flora of the Greater Sonoran Desert Region 1: 164 (2002)

Literature: Meesim and Mongkolsuk 2010a; Mongkolsuk and Meesim 2012 as Pyxine nubila

320. Dendriscosticta platyphylla (Trevis.) B.Moncada \& Lücking, Lichenologist 45: $222(2013)$

Literature: Satô 1962; Yoshimura 1978; Wolseley and Aguirre-Hudson 1995; 1997a; Wolseley et al., 2002 as Sticta nylanderiana

321. Dendriscosticta platyphylloides (Nyl.) B.Moncada \& Lücking, Lichenologist 45: 223 (2013)

Literature: Yoshimura 1978; Wolseley et al., 2002 as Sticta platyphylloides

322. Dendriscosticta wrightii (Tuck.) Moncada \& Lücking, Lichenologist 45: 223 (2013)

Literature: Vainio 1921; Wolseley et al., 2002 as Sticta wrightii

323. Dibaeis arcuata (Stirt.) Kalb \& Gierl, in Gierl \& Kalb, Herzogia 9: 617 (1993) Literature: Aptroot et al., 2007 as Baeomyces fungoides

324. Dichosporidium boschianum (Mont.) G.Thor, Opera Bot. 103: 64 (1991) Literature: Boonpragob et al., 1998; Wolseley et al., 2002

325. Dictyonema thelephora (Spreng.) Zahlbr., Cat. Lich. Univ. 7: 748 (1931) Literature: Vainio 1909; Paulson 1930 as Dictyonema sericeum; Wolseley and Aguirre-Hudson 1995; Wolseley et al., 2002 as Dictyonema sericeum $\mathrm{f}$. thelephora

326. Dimelaena tenuis (Müll.Arg.) H.Mayrhofer \& Wippel, in H.Mayrhoferofer et al., Mycotaxon 58: 304 (1996)

Literature: Aptroot et al., 2007; Mongkolsuk and Meesim 2012

327. Dimelaena thysanota (Tuck.) Hale \& W.L.Culb., Bryologist 73: 513 (1970)

Literature: Meesim and Mongkolsuk 2010b; Mongkolsuk and Meesim 2012

328. Dimelaena weberi Sheard, in Sheard \& H.Mayrhofer, Bryologist 87: 246 (1984)

Literature: Meesim and Mongkolsuk 2010b

329. Diorygma angusticarpum Sutjaritt. \& Kalb, Phytotaxa 189: 314 (2014)

Literature: Sutjaritturakan and Kalb 2014

330. Diorygma chumphonense Sutjaritt. \& Kalb, Phytotaxa 189: 314 (2014)

Literature: Sutjaritturakan and Kalb 2014

331. Diorygma citri Sutjaritt. \& Kalb, Phytotaxa 189: 316 (2014)

Literature: Sutjaritturakan and Kalb 2014 
332. Diorygma conprotocetraricum Sutjaritt. \& Kalb, Phytotaxa 189: 316 (2014) Literature: Sutjaritturakan and Kalb 2014

333. Diorygma erythrellum (Mont. \& Bosch) Kalb, Staiger \& Elix, Symb. Bot. Upsal. 34: 150 (2004)

Literature: Nakanishi et al., 2001 as Graphina erythrella

334. Diorygma fuscopruinosum Sutjaritt. \& Kalb, Phytotaxa 189: 317 (2014)

Literature: Sutjaritturakan and Kalb 2014

335. Diorygma hieroglyphicellum Sutjaritt. \& Kalb, Phytotaxa 189: 317 (2014)

Literature: Sutjaritturakan and Kalb 2014

336. Diorygma hieroglyphicum (Pers.) Staiger \& Kalb, Biblioth. Lichenol. 85: 113 (2002)

Literature: Aptroot et al., 2007 as Graphis albidolivens; Poengsungnoen et al., 2010; Mongkolsuk et al., 2013; as Graphis siamensis Vain., (Wolseley et al., 2002)

337. Diorygma inexpectatum Sutjaritt. \& Kalb, Phytotaxa 189: 318 (2014) Literature: Sutjaritturakan and Kalb 2014

338. Diorygma junghuhnii (Mont. \& Bosch) Kalb, Staiger \& Elix, Symb. Bot. Upsal. 34: 157 (2004)

Literature: Vainio 1921 as Graphis obtecta var. columbiana; Wolseley et al., 2002 as Graphina columbiana; Aptroot et al., 2007; Poengsungnoen et al., 2010

339. Diorygma pruinosum (Eschw.) Kalb, Staiger \& Elix, Symb. Bot. Upsal. 34: 166 (2004)

Literature: Aptroot et al., 2007

340. Diorygma reniforme (Fée) Kalb, Staiger \& Elix, Symb. Bot. Upsal. 34: 167 (2004)

Literature: Aptroot et al., 2007; Poengsungnoen et al., 2010

341. Diorygma salazinicum Sutjaritt. \& Kalb, Phytotaxa 189: 319 (2014)

Literature: Sutjaritturakan and Kalb 2014

342. Diorygma soozanum (Zahlbr.) M.Nakan. \& Kashiw., in Nakanishi et al., Bull. Natl. Sci. Mus., Tokyo 29: 86 (2003)

Literature: Kalb et al., 2009b

343. Diorygma subpruinosum Sutjaritt. \& Kalb, Phytotaxa 189: 319 (2014)

Literature: Sutjaritturakan and Kalb 2014

344. Diorygma thailandicum Sutjaritt. \& Kalb, Phytotaxa 189: 321 (2014)

Literature: Sutjaritturakan and Kalb 2014

345. Diploschistes actinostomus (Ach.) Zahlbr., Hedwigia 31: 34 (1892)

Literature: Aptroot et al., 2007

346. Diploschistes cinereocaesius (Sw. ex Ach.) Vain., Ann, Acad. Sci. Fenn., ser. A 15(6): 172 (1921)

347. Diplotomma alboatrum (Hoffm.) Flot., Jahrsb. Schles. Ges. Vaterl. Kult. 27: 97 (1849)

Literature: Mongkolsuk and Meesim 2012 
348. Diplotomma venustum (Körb.) Körb., Parerga Lichenol.: 179 (1860)

Literature: Meesim and Mongkolsuk 2010b as Buellia venusta

349. Dirinaria aegialita (Ach.) B.J.Moore, Bryologist 71: 248 (1968)

Literature: Wolseley and Aguirre-Hudson 1997a; Wolseley et al., 2002; Meesim and Mongkolsuk 2010a; Mongkolsuk and Meesim 2012; Van den Boom et al., 2014

350. Dirinaria applanata (Fée) D.D.Awasthi, J. Indian Bot. Soc. 49:135 (1970)

Literature: Wolseley and Aguirre-Hudson 1997a; Wolseley et al., 2002; Saipunkaew et al., 2007; Meesim and Mongkolsuk 2010a; Mongkolsuk and Meesim 2012; Schumm and Aptroot 2012; Van den Boom et al., 2014

351. Dirinaria confluens (Fr.) D.D.Awasthi, Biblioth. Lichenol. 2: 28 (1975)

Literature: Aptroot et al., 2007; Meesim and Mongkolsuk 2010a; Mongkolsuk and Meesim 2012

352. Dirinaria confusa D.D.Awasthi, Bull. Soc. Bot. France, Lett. Bot. 121: 56 (1975)

Literature: Yoshimura 1978; Wolseley et al., 2002

353. Dirinaria consimilis (Stirt.) D.D.Awasthi, in Awasthi \& Agarwal, J. Indian Bot. Soc. 49: 135 (1970)

Literature: Wolseley and Aguirre-Hudson 1995; 1997a; 1997b; Wolseley et al., 2002; Saipunkaew et al., 2007

354. Dirinaria papillulifera (Nyl.) D.D.Awasthi, Bryologist 67: 369 (1964)

Literature: Aptroot et al., 2007; Van den Boom et al., 2014

355. Dirinaria picta (Sw.) Schaer. ex Clem., in Clements \& Shear, Gen. Fungi, ed. 2.: 323 (1931)

Literature: Paulson 1930; Vainio 1909 as Physcia picta; Wolseley and AguirreHudson 1995; Wolseley et al., 2002; Papong et al., 2007; Saipunkaew et al., 2007; Meesim and Mongkolsuk 2010a; Schumm and Aptroot 2012; Mongkolsuk and Meesim 2012; Mongkolsuk et al., 2013

356. Dirinaria purpurascens (Vain.) B.J.Moore, Bryologist 71: 251 (1968)

Literature: Aptroot et al., 2007

357. Dyplolabia afzelii (Ach.) A.Massal., Neagen. Lich.: 6 (1854)

Literature: Boonpragob et al., 1998; Wolseley et al., 2002; Poengsungnoen et al., 2010; Mongkolsuk et al., 2012; Mongkolsuk and Poengsungnoen 2013 as Graphis afzelii

358. Dyplolabia ochrocheila (Vain.) Rivas Plata \& Lücking, in Kalb, Polyiam, Rivas Plata, Bawingan, Kalb \& Lücking, Phytotaxa 268: 120 (2016)

Literature: Kalb et al., 2016b

359. Dyplolabia chumphonensis J. Kalb \& K. Kalb, in Kalb, Polyiam, Rivas Plata, Bawingan, Kalb \& Lücking, Phytotaxa 268(2): 115 (2016)

Literature: Kalb et al., 2016b

360. Echinoplaca epiphylla Fée, Essai Crypt. Écorc.: 93 (1825) [1824]

Literature: Boonpragob et al., 1998; Wolseley et al., 2002

361. Echinoplaca leucotrichoides (Vain.) R.Sant., in Thorold, J. Ecol. 40: 129 (1952) 
Literature: Aptroot et al., 2007

362. Echinoplaca pellicula (Müll.Arg.) R.Sant., Symb. Bot. Upsal. 12: 367 (1952)

Literature: Boonpragob et al., 1998; Wolseley et al., 2002

363. Echinoplaca tetrapla (Zahlbr.) Lücking, Lichenologist 33: 203 (2001)

Literature: Papong et al., 2007

364. Endocarpon adscendens (Anzi) Müll.Arg., Bull. Murith. Soc. Valais. Sci. Nat. 10: 58 (1881)

Literature: Boonpragob et al., 1998; Wolseley et al., 2002

365. Endocarpon pallidulum (Nyl.) Nyl., in Hue, Nouv. Arch. Mus. Hist. Nat. 4: 106 (1892)

Literature: Aptroot et al., 2007

366. Enterographa anguinella (Nyl.) Redinger, Reprium nov. Spec. Regni veg. 43: 62 (1938)

Literature: Aptroot et al., 2007

367. Enterographa angustissima (Vain.) R.Sant., Symb. Bot. Upsal. 12: 105 (1952) Literature: Papong et al., 2007

368. Enterographa inthanonensis Sparrius, in Aptroot et al., Fungal Diversity 24: 98 (2007)

Literature: Aptroot et al., 2007

369. Enterographa mesomela Sparrius, Saipunkaew \& Wolseley, Lichenologist 38: 30 (2006)

Literature: Sparrius et al., 2006

370. Enterographa pallidella (Nyl.) Redinger, Reprium nov. Spec. Regni veg. 43: 61 (1938)

Literature: Vainio 1909 as Enterographa pallidellum var. olivaceoalba, Chiodecton pallidellum var. perpallida; Wolseley et al., 2002 as Chiodecton pallidellum var. olivaceoalba, Chiodecton pallidellum var. olivaceostraminea, Chiodecton pallidellum var. perpallida; Schumm and Aptroot 2012

371. Enterographa subserialis (Nyl.) Redinger, Reprium nov. Spec. Regni veg. 43: 69 (1938)

Literature: Aptroot et al., 2007

372. Enterographa tropica Sparrius, Biblioth. Lichenol. 89: 61 (2004)

Literature: Aptroot et al., 2007

373. Eremothecella calamicola Syd. \& P.Syd., Ann. Mycol. 15: 236 (1917)

Literature: Aptroot et al., 2007

374. Eremothecella macrocephala (R.Sant.) Thor, Lücking \& Matsumoto, Symb. Bot. Upsal. 32: 39 (2000)

Literature: Boonpragob et al., 1998 as Stirtonia macrocephala; Wolseley et al., 2002

375. Erioderma sorediatum D.J.Galloway \& P.M.Jørg., Lichenologist 7: 139 (1975) Literature: Wolseley and Aguirre-Hudson 1995; Boonpragob et al., 1998; Wolseley et al., 2002

376. Eschatogonia marivelensis (Vain.) Kalb, Biblioth. Lichenol. 88: 310 (2004) 
Literature: Kalb et al., 2009a

377. Eschatogonia prolifera (Mont.) R.Sant., in Swinscow \& Krog, Macrolichens East Africa: 86 (1988)

Literature: Boonpragob et al., 1998; Wolseley et al., 2002; Aptroot et al., 2007; Kalb et al., 2012

378. Eugeniella micrommata (Kremp.) Lücking, Sérus. \& Kalb, Fl. Neotrop. Monogr. 103: 716 (2008)

Literature: Papong et al., 2007 as Bacidia micrommata; Aptroot et al., 2007

379. Fellhanera bouteillei (Desm.) Vězda, Folia Geobot. Phytotax. 21: 214 (1986)

Literature: Aptroot et al., 2007; Papong et al., 2007

380. Fellhanera fuscatula (Müll.Arg.) Vězda, Folia Geobot. Phytotax. 21: 214 (1986) Literature: Aptroot et al., 2007

381. Fellhanera mastothallina (Vain.) Lücking \& Sérus., Lichenologist 33: 192 (2001) Literature: Papong et al., 2007

382. Fellhanera rhaphidophylli (Rehm) Vězda, Folia Geobot. Phytotax. 21: 214 (1986)

Literature: Papong et al., 2007

383. Fibrillithecis argentea (Müll.Arg.) Rivas Plata \& Lücking, in Rivas Plata, Lücking , Sipman, Mangold, Kalb \& Lumbsch, Lichenologist 42: 183 (2010)

Literature: Wolseley et al., 2002 as Thelotrema piluliferum and Thelotrema platysporum

384. Fissurina albonitens (Müll.Arg.) A.W.Archer, Telopea 11: 71 (2005)

Literature: Paulson 1930 as Graphis albonitens

385. Fissurina cingalina (Nyl.) Staiger, Biblioth. Lichenol. 85: 128 (2002)

Literature: Poengsungnoen et al., 2010

386. Fissurina dumastii Fée, Essai Crypt. Écorc.: 90 (1825) [1824]

Literature: Aptroot et al., 2007; Lumbsch et al., 2014

387. Fissurina incrustans Müll.Arg., Mém. Soc. Phys. Genève 29: 45 (1887)

Literature: Aptroot et al., 2007

388. Fissurina inabensis Vain., Bot. Mag., Tokyo 32: 161 (1918)

Literature: Nakanishi et al., 2001 as Graphina inabensis

389. Fissurina inquinata C.Knight \& Mitt., Trans. Linn. Soc. London 23: 102 (1860)

Literature: Aptroot et al., 2007

390. Fissurina insculpta Mont., Ann. Sci. Nat., Bot. 18: 280 (1842)

Literature: Aptroot et al., 2007

391. Fissurina quadrispora Kalb, Herzogia 9: 64 (1992)

Literature: Aptroot et al., 2007

392. Fissurina marginata Staiger, Biblioth. Lichenol. 85: 144 (2002)

Literature: Lumbsch et al., 2014

393. Fissurina niveoalba Poengs. \& Kalb, Phytotaxa 189: 255-267 (2014)

Literature: Poengsungnoen et al., 2014b

394. Fissurina phuluangii Poengs. \& Kalb, Phytotaxa 189: 255-267 (2014) 
Literature: Poengsungnoen et al., 2014b

395. Fissurina pseudostromatica Lücking \& Rivas Plata, Bull. Florida Mus. Nat. Hist. 49: 145 (2011)

Literature: Lumbsch et al., 2014

396. Fissurina radiata Mont., Ann. Sci. Nat., Bot. 18: 280 (1842)

Literature: Aptroot et al., 2007

397. Fissurina rubiginosa (Fée) Staiger, Biblioth. Lichenol. 85: 148 (2002)

Literature: Aptroot et al., 2007

398. Fissurina rufula (Mont.) Staiger, Biblioth. Lichenol. 85: 160 (2002)

Literature: Aptroot et al., 2007

399. Flakea papillata O.E.Erikss., Syst. Ascomycetum 11: 14 (1992)

Literature: Aptroot et al., 2007 as Agonimia papillata

400. Flavoparmelia caperata (L.) Hale, Mycotaxon 25: 604 (1986)

Literature: Wolseley and Aguirre-Hudson 1995 as Pseudoparmelia caperatum

401. Fuscopannaria siamensis P.M.Jørg. \& Wolseley, J. Hattori Bot. Lab. 89: 256 (2000)

Literature: Jørgensen 2000; Wolseley et al., 2002

402. Gassicurtia chermesina (Kalb) Marbach, Biblioth. Lichenol. 74: 222 (2000)

Literature: Aptroot et al., 2007

403. Gassicurtia clathrisidiata Aptroot, in Aptroot et al., Fungal Diversity 24: 101 (2007)

Literature: Aptroot et al., 2007

404. Gassicurtia coccifera Marbach \& Kalb, in Marbach, Biblioth. Lichenol. 74: 223 (2000)

Literature: Van den Boom et al., 2014

405. Gassicurtia omiae Kalb, in Kalb, Archer, Sutjaritturakan \& Boonpragob, Biblioth. Lichenol. 99: 230 (2009)

Literature: Kalb et al., 2009a

406. Gibbosporina boninensis (Kurok.) Elvebakk \& P.M.Jørg., Lichenologist 48: 29 (2016)

Literature: Aptroot et al., 2007 as Psoroma sphinctrinum

407. Glaucotrema costaricense (Müll.Arg.) Rivas Plata \& Lumbsch, Taxon 6: 1175 (2013) Literature: Boonpragob et al., 1998; Wolseley et al., 2002 as Myriotrema costaricense

408. Glaucotrema glaucophaenum (Kremp.) Rivas Plata \& Lumbsch, Taxon 61: 1175 (2012)

Literature: Mongkolsuk et al., 2013 as Myriotrema glaucophaenum; Kraichak et al., 2014; Papong et al., 2014

409. Glaucotrema thailandicum Naksuwankul, Lücking \& Lumbsch, MycoKeys 17: $53(2016)$

Literature: Naksuwankul et al., 2016

410. Glyphis cicatricosa Ach., Syn. Meth. Lich.: 107 (1814)

Literature: Vainio 1909; Wolseley et al., 2002; Poengsungnoen et al., 2010 as Graphis cicatricosa var. confluens 
411. Glyphis scyphulifera (Ach.) Staiger, Biblioth. Lichenol. 85: 175 (2002)

Literature: Vainio 1909; Wolseley and Aguirre-Hudson 1997a; Boonpragob et al., 1998; Wolseley et al., 2002 as Gyrostomum scyphuliferum; Schumm and Aptroot 2012

412. Graphidastra byssiseda (Müll.Arg.) G.Thor, Opera Bot. 103: 80 (1991)

Literature: Wolseley and Aguirre-Hudson 1997a; Wolseley et al., 2002

413. Graphidastra laii Aptroot \& Sparrius, Lichenologist 38: 31 (2006)

Literature: Sparrius et al., 2006

414. Graphis acharii Fée, Essai Crypt. Écorc.: 39 (1825) [1824]

Literature: Aptroot et al., 2007

415. Graphis analoga Nyl., Ann. Sci. Nat., Bot. 11: 244 (1859)

Literature: Vainio 1909; Alava 1988; Wolseley et al., 2002 as Graphina simplex; Pitakpong et al., 2015

416. Graphis arbusculiformis (Vain.) Lücking, in Lücking, Sutjaritturakan \& Kalb, Lichenologist 44(3): 391 (2012)

Literature: Lücking et al., 2012

417. Graphis assimilis Nyl., Bull. Soc. Linn. Normandie 2: 109 (1868)

Literature: Poengsungnoen et al., 2010

418. Graphis australosiamensis Sutjaritt. \& Kalb, Phytotaxa 189: 321 (2014)

Literature: Sutjaritturakan and Kalb 2014

419. Graphis caesiella Vain., Acta Soc. Fauna Fl. Fenn. 7: 122 (1890)

Literature: Pitakpong et al., 2015

420. Graphis cincta (Pers.) Aptroot, Flora of Australia (Melbourne) 57: 651 (2009) Literature: Pitakpong et al., 2015

421. Graphis concolor Nyl., Mém. Soc. Sci. nat. Cherbourg 5: 129 (1857)

Literature: Vainio 1909; Wolseley et al., 2002 as Graphina boschiana

422. Graphis consimilis Vain., Hedwigia 46: 177 (1907)

Literature: Vainio 1909; Alava 1988; Wolseley et al., 2002 as Graphina consimilis

423. Graphis dendrogramma Nyl. in Lücking et al., Lichenologist 41: 363-452 (2009)

Literature: Papong et al., 2007 as Graphis ceylanica

424. Graphis dussii Vain., Hedwigia 38: 255 (1899)

Literature: Aptroot et al., 2007

425. Graphis emersa Müll.Arg., Hedwigia 32: 132 (1893)

Literature: Poengsungnoen et al., 2010

426. Graphis fissurinoidea (Nyl.) Vain., Bot. Tidsskr. 29: 128 (1909)

Literature: Vainio 1909; Wolseley et al., 2002 as Graphina fissurinoidea

427. Graphis furcata Fée, Essai Crypt. Écorc.: 40 (1825) [1824]

Literature: Poengsungnoen et al., 2010; Pitakpong et al., 2015

428. Graphis glaucescens Fée, Essai Crypt. Écorc.: 36 (1825) [1824]

Literature: Aptroot et al., 2007

429. Graphis glaucocinerea Vain., Hedwigia 46: 178 (1907)

Literature: Vainio 1909 as Graphina glaucocinerea; Wolseley et al., 2002 
430. Graphis glaucorufa Vain., Ann. Bot. Soc. Zool.-Bot. Fenn. "Vanamo" 1(no. 3): 51 (1921)

Literature: Vainio 1921; Alava 1988; Nakanishi et al., 2001; Wolseley et al., 2002 as Graphina glaucorufa

431. Graphis handelii Zahlbr., in Handel-Mazzetti, Symb. Sin. 3: 44 (1930)

Literature: Poengsungnoen 2010 as Graphis chondroplaca; Pitakpong et al., 2015

432. Graphis hossei Vain., Ann. Bot. Soc. Zool.-Bot. Fenn. "Vanamo" 1: 53 (1921) Literature: Vainio 1921; Alava 1988; Wolseley et al., 2002 as Graphis tenuis; Poengsungnoen et al., 2010

433. Graphis intermedians Vain., Ann. Bot. Soc. Zool.-Bot. Fenn. "Vanamo" 1: 50 (1921)

Literature: Vainio 1921; Wolseley et al., 2002 as Graphina intermedians

434. Graphis irosina Vain. Ann. Acad. Sci. fenn., ser. A 15: 213 (1921)

Literature: Nakanishi et al., 2001 as Graphina irosina

435. Graphis jejuensis K.H. Moon, M. Nakan. \& Kashiw., Jap. J. Bot. 87: 320-325 (2012)

Literature: Pitakpong et al., 2015

436. Graphis koratensis Pitakpong, Kraichak, Lücking, Lichenologist 47: 338 (2015) Literature: Pitakpong et al., 2015

437. Graphis leptospora Vain., Ann. Bot. Soc. Zool.-Bot. Fenn. "Vanamo" 1: 53 (1921)

Literature: Vainio 1921; Wolseley et al., 2002

438. Graphis librata C.Knight, Trans. \& Proc. New Zealand Inst. 16: 404 (1884) [1883]

Literature: Poengsungnoen et al., 2010; Schumm and Aptroot 2012; Pitakpong et al., 2015

439. Graphis lineola Ach., Lichenogr. Universalis: 264 (1810)

Literature: Vainio 1909; Wolseley et al., 2002 as Graphina lineola

440. Graphis longiramea Müll.Arg., J. Linn. Soc., Bot. 29: 225 (1892)

Literature: Poengsungnoen et al., 2010

441. Graphis longispora D.D.Awasthi \& S.R.Singh, Norweg. J. Bot. 24: 3 (1977)

Literature: Poengsungnoen et al., 2010

442. Graphis lumbricina Vain., Hedwigia 38: 256 (1899)

Literature: Aptroot et al., 2007

443. Graphis marginata Raddi, Atti Soc. Naturalisti Mat. Modena 18: 344 (1820)

Literature: Poengsungnoen et al., 2010

444. Graphis nanodes Vain., Ann. Acad. Sci. Fenn., Ser. A, 15: 209 (1921)

Literature: Poengsungnoen et al., 2010

445. Graphis nigrocarpa Adaw. \& Makhija, Mycotaxon 96: 56 (2006)

Literature: Pitakpong et al., 2015

446. Graphis norvestitoides Sutjaritturakan, Lichenologist 44(3): 391 (2012)

Literature: Lücking et al., 2012 
447. Graphis novopalmicola A.W. Archer \& Lücking, in Lücking, Archer \& Aptroot, Lichenologist 41(4): 439 (2009)

Literature: Poengsungnoen et al., 2010

448. Graphis nuda (H.Magn.) Staiger \& Lücking, in Lücking, Chaves, Sipman, Umaña \& Aptroot, Fieldiana, Bot. 38: 93 (2008)

Literature: Vainio 1909; Wolseley et al., 2002 as Graphina ruiziana var. gracilior

449. Graphis ochrocheila Vain., Hedwigia 46: 178 (1907)

Literature: Vainio 1909 as Graphina chrocheila; Wolseley et al., 2002

450. Graphis pavoniana Fée, Essai Crypt. Écorc.: 40 (1825) [1824]

Literature: Aptroot et al., 2007; Pitakpong et al., 2015

451. Graphis persimilis Vain., Bot. Tidsskr. 29: 125 (1909)

Literature: Wolseley et al., 2002 as Phaeographina persimilis

452. Graphis perstriatula Nyl., Bull. Soc. Linn. Normandie, sér. 2, 7: 176 (1873)

Literature: Nakanishi et al., 2001 as Graphina perstriatula

453. Graphis phaeospora Vain. in Lücking et al., Lichenologist 41: 363-452 (2009). Literature: Aptroot et al., 2007 as Graphis subcinerea; Pitakpong et al., 2015

454. Graphis proserpens Fée, Essai Crypt. Écorc.: 40 (1825) [1824]

Literature: Aptroot et al., 2007; Pitakpong et al., 2015

455. Graphis pyrrhocheiloides Zahlbr., Cat. Lich. Univ. 2: 321 (1923) [1924]

Literature: Alava 1988 as Graphina pyrrhocheila; Wolseley et al., 2002; Poengsungnoen 2010; Pitakpong et al., 2015

456. Graphis renschiana (Müll. Arg.) Stizenb., Ber. Tätigk. St. Gallischen Naturwiss. Ges.: 184 (1891)

Literature: Pitakpong et al., 2015

457. Graphis rhizocola (Fée) Lücking \& Chaves, in Lücking et al., Fieldiana, Bot. 46: 102 (2008)

Literature: Poengsungnoen et al., 2010; Pitakpong et al., 2015

458. Graphis rimulosa (Mont.) Trevis., Spighe e Paglie: 11 (1853)

Literature: Poengsungnoen et al., 2010; Pitakpong et al., 2015

459. Graphis rongklaensis Sutjaritturakan Lücking 44(3): 391-394 (2012)

Literature: Lücking et al. 2012; Nakanishi et al., 2001 as Graphina vestita

460. Graphis rustica Kremp., Nuovo Giorn. Bot. Ital. 7: 61 (1875)

Literature: Poengsungnoen et al., 2010

461. Graphis sauroidea Leight., Trans. Linn. Soc. London 25: 452 (1866)

Literature: Vainio 1909 as Graphina sauroidea; Wolseley et al., 2002

462. Graphis schmidtii Vain., Hedwigia 46: 176 (1907)

Literature: Vainio 1909; Wolseley et al., 2002 as Phaeographina schmidtii

463. Graphis seminuda Müll. Arg., Bull. Soc. Roy. Bot. Belgique 30: 79 (1891)

Literature: Pitakpong et al., 2015

464. Graphis semirigida (Müll.Arg.) Lücking, Lichenologist 41: 422 (2009)

Literature: Poengsungnoen et al., 2010

465. Graphis stenotera Vain., Ann. Acad. Sci. Fenn., Ser. A, 15: 243 (1921)

Literature: Poengsungnoen et al., 2010 
466. Graphis streblocarpa (Bél.) Müll. Arg., Flora, Regensburg 65: 502 (1882) Literature: Vainio 1921; Wolseley et al., 2002 as Graphina streblocarpa var. pauperior Poengsungnoen et al., 2010; Pitakpong et al., 2015

467. Graphis striatula (Ach.) Spreng., Syst. Veg. (ed. 16) [Sprengel] 4: 250 (1827) Literature: Vainio 1909 as Graphina striatula; Wolseley et al., 2002

468. Graphis subdisserpens Nyl., Bull. Soc. Linn. Normandie 7: 175 (1873) Literature: Vainio 1909 as Graphina subdisserpens; Wolseley et al., 2002

469. Graphis subdussii Poengs. \& Kalb, Phytotaxa 189: 255-267 (2014) Li te r ature: Poengsungnoen et al., 2014b

470. Graphis subinsulana Poengs. \& Kalb, Phytotaxa 189: 255-267 (2014)

Literature: Poengsungnoen et al., 2014b

471. Graphis subobtecta var. feracior Vain., Ann. Bot. Soc. Zool.-Bot. Fenn. "Vanamo" 1(no. 3): 51 (1921)

Literature: Vainio 1921; Alava 1988; Wolseley et al., 2002 as Graphina subobtecta var. feracior

472. Graphis subrigida Nyl., J. Linn. Soc., Bot. 20: 58 (1883)

Literature: Vainio 1909; Wolseley et al., 2002 as Phaeographina subrigida

473. Graphis subserpentina Nyl., Acta Soc. Sci. Fenn. 7(2): 465 (1863)

Literature: Pitakpong et al., 2015; Nakanishi et al., 2001; Poengsungnoen et al., 2010 as Graphina subserpentina

474. Graphis subregularis A.W.Archer, Austral. Syst. Bot. 14: 266 (2001)

Literature: Poengsungnoen et al., 2010

475. Graphis subvittata Adaw. \& Makhija, Mycotaxon 99: 322 (2007)

Literature: Poengsungnoen et al., 2010

476. Graphis supracola A.W.Archer, Aust. Syst. Bot. 14: 267 (2001)

Literature: Poengsungnoen et al., 2010; Schumm and Aptroot 2012

477. Graphis tenella var. epiphaea Vain., Bot. Tidsskr. 29: 133 (1909)

Literature: Vainio 1909 and Alava 1988 as Graphina tenella var. epiphaea; Wolseley et al., 2002

478. Graphis tenella Ach., Syn. Meth. Lich.: 81 (1814)

Literature: Vainio 1921; Wolseley et al., 2002; Pitakpong et al., 2015

479. Graphis trichospora Vain., Ann. Bot. Soc. Zool.-Bot. Fenn. "Vanamo" 1: 52 (1921) Literature: Vainio 1921; Wolseley et al., 2002

480. Graphis verminosa Müll.Arg., J. Linn. Soc., Bot. 29: 224 (1892)

Literature: Poengsungnoen et al., 2010

481. Graphis vittata Müll.Arg., Flora 65: 335 (1882)

Literature: Poengsungnoen et al., 2010

482. Gyalectidium caucasicum (Elenkin \& Woron.) Vězda, Folia Geobot. Phytotax. 18: 56 (1983)

Literature: Aptroot et al., 2007

483. Gyalectidium novoguineense Sérus., in Ferraro et al., J. Linn. Soc., Bot. 137: $340(2001)$

Literature: Papong et al., 2007 
484. Gyalidea luzonensis (Kalb \& Vězda) Aptroot \& Lücking, Biblioth. Lichenol. 86: 69 (2003)

Literature: Aptroot et al., 2007

485. Gyalideopsis altamirensis Lücking \& Umaña, in Lücking et al., Lichenologist 38: 133 (2006)

Literature: Wolseley et al., 2002; Aptroot et al., 2007

486. Gyalideopsis lambinonii Vězda, Folia Geobot. Phytotax. 14: 64 (1979)

Literature: Aptroot et al., 2007

487. Gyalideopsis rubescens Vězda, Folia Geobot. Phytotax. 14: 67 (1979)

Literature: Boonpragob et al., 1998

488. Gyalideopsis vainioi Kalb \& Vězda, Biblioth. Lichenol. 29: 51 (1988)

Literature: Aptroot et al., 2007

489. Gymnoderma coccocarpum Nyl., Syn. Meth. Lich. 2: 26 (1863)

Literature: Wolseley and Aguirre-Hudson 1995; Wolseley et al., 2002

490. Gymnographa heterospora (Nyl.) Staiger, Biblioth. Lichenol. 85: 271 (2002) Literature: Aptroot et al., 2007

491. Haematomma africanum (J. Steiner) C.W. Dodge, Beih. Nova Hedwigia 38: 39 (1971)

Literature: Sriprang and Mongkolsuk 2010

492. Haematomma collatum (Stirt.) C.W.Dodge, Beih. Nova Hedwigia 38: 41 (1971)

Literature: Wolseley et al., 2002; Sriprang and Mongkolsuk 2010

493. Haematomma flexuosum Hillmann, Feddes Repert. Spec. Nov. Regni Veg. 49: 35 (1938)

Literature: Kalb et al., 2009a

494. Haematomma parda Aptroot, in Aptroot et al., Fungal Diversity 24: 103 (2007)

Literature: Aptroot et al., 2007

495. Haematomma puniceum (Sm. ex Ach.) A.Massal, Atti Ist. Veneto Sci. Lett. Arti 5: 253. (1860)

Literature: Vainio 1921; Wolseley et al., 1994; Wolseley and Aguirre-Hudson 1997b; Wolseley et al., 2002

496. Haematomma rufidulum (Fée) A.Massal., Atti Ist. Veneto Sci. Lett. Arti 5: 253 (1860) [1859-1860]

Literature: Aptroot et al., 2007; Kalb et al., 2009a; Sriprang and Mongkolsuk 2010

497. Haematomma wattii (Stirt.) Zahlbr., Cat. Lich. Univ. 5: 776 (1928)

Literature: Wolseley and Aguirre-Hudson 1997a; Wolseley et al., 2002

498. Heiomasia sipmanii (Aptroot, Lücking \& Rivas Plata) Nelsen, Lücking \& Rivas Plata, Bryologist 113: 748 (2010) Literature: Aptroot et al., 2009, as Herpothallon sipmanii, Nelson et al., 2010

499. Hemithecium aphanes Mont. \& Bosch, in Miquel, Pl. Jungh. 4: 474 (1855) Literature: Nakanishi et al., 2001 as Graphis aphanes 
500. Hemithecium implicatum (Fée) Staiger, Biblioth. Lichenol. 85: 287 (2002)

Literature: Pitakpong et al., 2015 as Graphis implicata

501. Herpothallon confluenticum Aptroot \& Lücking, in Aptroot et al., Biblioth. Lichenol. 99: 35 (2009)

Literature: Aptroot et al., 2009

502. Herpothallon echinatum Aptroot, Lücking \& Will-Wolf, in Aptroot et al., Biblioth. Lichenol. 99: 38 (2009)

Literature: Aptroot et al., 2009

503. Herpothallon granulare (Sipman) Aptroot \& Lücking, in Aptroot et al., Biblioth. Lichenol. 99: 43 (2009)

Literature: Aptroot et al., 2007 as Cryptothecia granularis; Aptroot et al., 2009

504. Herpothallon philippinum (Vain.) Aptroot \& Lücking, in Aptroot et al., Biblioth. Lichenol. 99: 56 (2009)

Literature: Aptroot et al., 2009

505. Heterocyphelium leucampyx (Tuck.) Vain., Acta Soc. Fauna Fl. Fenn. 57: 16 (1927) Literature: Aptroot et al., 2007

506. Heterodermia angustiloba (Müll.Arg.) D.D.Awasthi, Geophytology 3(1): 113 (1973)

Literature: Mongkolsuk et al., 2015

507. Heterodermia antillarum (Vain.) Swinscow \& Krog, Lichenologist 8: 114 (1976)

Literature: Aptroot et al., 2007; Meesim and Mongkolsuk 2010a; Mongkolsuk and Meesim 2012; Mongkolsuk et al., 2015

508. Heterodermia archeri Elix, Australas. Lichenol. 68: 16 (2011)

Literature: Mongkolsuk et al., 2015

509. Heterodermia comosa (Eschw.) Follmann \& Redón, Willdenowia 6: 446 (1972) Literature: Aptroot et al., 2007; Meesim and Mongkolsuk 2010a; Mongkolsuk and Meesim 2012; Mongkolsuk et al., 2015

510. Heterodermia coralloidea Elix, Australas. Lichenol. 69: 12 (2011)

Literature: Mongkolsuk et al., 2015

511. Heterodermia dactyliza (Nyl.) Swinscow \& Krog, Lichenologist 8: 117 (1976) Literature: Meesim and Mongkolsuk 2010a

512. Heterodermia diademata (Taylor) D.D.Awasthi, Geophytology 3: 113 (1973)

Literature: Wolseley and Aguirre-Hudson 1997b; Wolseley et al., 2002; Meesim and Mongkolsuk 2010a; 2012; Van den Boom et al., 2014; Mongkolsuk et al., 2015

513. Heterodermia dissecta (Kurok.) D.D.Awasthi, Geophytology 3: 113 (1973)

Literature: Mongkolsuk et al., 2015

514. Heterodermia esorediata (Vain.) Du Rietz \& Lynge, Vidensk. I. Mat.-Nat. Kl. 16: 14 (1924)

Literature: Wolseley et al., 2002

515. Heterodermia firmula (Nyl.) Trevis., Atti Soc. Ital. Sci. Nat. 11: 615 (1868) Literature: Mongkolsuk et al., 2015 
516. Heterodermia flabellata (Fée) D.D.Awasthi, Geophytology 3: 113 (1973)

Literature: Aptroot et al., 2007; Meesim and Mongkolsuk 2010a; Mongkolsuk and Meesim 2012; Van den Boom et al., 2014; Mongkolsuk et al., 2015

517. Heterodermia flavosquamosa Aptroot \& Sipman, in Sipman, Willdenowia 19: $546(1990)$

Literature: Meesim and Mongkolsuk 2010a; Mongkolsuk and Meesim 2012

518. Heterodermia fragilissima (Kurok.) J.C.Wei \& Y.M.Jiang, [Lichens of Xizang] (China): 111 (1986)

Literature: Wolseley and Aguirre-Hudson 1997a; Wolseley et al., 2002

519. Heterodermia galactophylla (Tuck.) W.L.Culb., Bryologist 69: 482 (1967)

Literature: Aptroot et al., 2007; Mongkolsuk et al., 2015

520. Heterodermia hypochraea (Vain.) Swinscow \& Krog, Lichenologist 8: 119 (1976) Literature: Van den Boom et al., 2014; Mongkolsuk et al., 2015

521. Heterodermia incana (Stirt.) D.D.Awasthi, Geophytology 3: 114 (1973) Literature: Wolseley et al., 2002; Mongkolsuk et al., 2015

522. Heterodermia isidiophora (Vain.) D.D.Awasthi, Geophytology 3: 114 (1973) Literature: Aptroot et al., 2007; Mongkolsuk et al., 2015

523. Heterodermia koyana (Kurok.) Elix, Australas. Lichenol. 66: 61 (2010)

Literature: Mongkolsuk et al., 2015

524. Heterodermia koyanoides Elix, Australas. Lichenol. 69: 14 (2011)

Literature: Mongkolsuk et al., 2015

525. Heterodermia lepidota Swinscow \& Krog, Lichenologist 8: 122 (1976)

Literature: Meesim and Mongkolsuk 2010a; Mongkolsuk and Meesim 2012

526. Heterodermia obscurata (Nyl.) Trevis., Nuovo Giorn. Bot. Ital. 1: 114 (1869)

Literature: Aptroot et al., 2007; Meesim and Mongkolsuk 2010a; Mongkolsuk and Meesim 2012; Mongkolsuk et al., 2015

527. Heterodermia pacifica (Kurok.) Kurok., Folia Cryptog. Estonica. 32: 23 (1998) Literature: Meesim and Mongkolsuk 2010a; Mongkolsuk and Meesim 2012

528. Heterodermia palpebrata (Taylor) Trass, Folia Cryptog. Estonica. 29: 17 (1992) Literature: Schumm and Aptroot 2012

529. Heterodermia pandurata (Kurok.) J.C.Wei, An enumeration of lichens in China: 112 (1991)

Literature: Wolseley et al., 2002; Mongkolsuk et al., 2015

530. Heterodermia paradoxa Schumm \& Schäf.-Verwimp, Herzogia 19: 36 (2006)

Literature: Schumm and Aptroot 2012; Mongkolsuk et al., 2015

531. Heterodermia podocarpa (Bél.) D.D.Awasthi, Geophytology 3: 114 (1973)

Literature: Aptroot et al., 2007; Meesim and Mongkolsuk 2010a; Mongkolsuk and Meesim 2012; Mongkolsuk et al., 2015

532. Heterodermia pseudospeciosa (Kurok.) W.L.Culb., Bryologist 69: 484 (1967) Literature: Wolseley and Aguirre-Hudson 1995; Meesim and Mongkolsuk 2010a; Mongkolsuk and Meesim 2012; Mongkolsuk et al., 2015

533. Heterodermia rubescens (Räsänen) D.D.Awasthi, Geophytology 3: 114 (1973) Literature: Mongkolsuk et al., 2015 
534. Heterodermia speciosa (Wulfen) Trevis., Atti. Soc. Ital. Sci. Nat. 11: 614 (1869) Literature: Vainio 1921 as Anaptychia speciosa; Wolseley et al., 2002; Meesim and Mongkolsuk 2010a; Mongkolsuk and Meesim 2012; Van den Boom et al., 2014; Mongkolsuk et al., 2015

535. Heterodermia stellata (Vain.) W.A.Weber, Mycotaxon 13: 102 (1981)

Literature: Meesim and Mongkolsuk 2010a; Mongkolsuk and Meesim 2012; Mongkolsuk et al., 2015

536. Heterodermia verdonii Elix, Australas. Lichenol. 68: 18 (2011)

Literature: Mongkolsuk et al., 2015

537. Hymenelia lacustris (With.) M.Choisy, Bull. Mens. Soc. Linn. Soc. Bot. Lyon 18: 145 (1949)

Literature: Aptroot et al., 2007

538. Hyperphyscia adglutinata (Flörke) H.Mayrhofer \& Poelt, Herzogia 5: 62 (1979) Literature: Saipunkaew et al., 2007; Meesim and Mongkolsuk 2010a; Mongkolsuk and Meesim 2012; Schumm and Aptroot 2012

539. Hyperphyscia cochlearis Scutari, Mycotaxon 61: 94 (1997)

Literature: Saipunkaew et al., 2007

540. Hyperphyscia granulata (Poelt) Moberg, Nordic J. Bot. 7: 721 (1987)

Literature: Aptroot et al., 2007

541. Hyperphyscia pandani (H.Magn.) Moberg, Nordic J. Bot. 7: 722 (1987)

Literature: Saipunkaew et al., 2007

542. Hypoflavia crustosa Aptroot, in Aptroot et al., Fungal Diversity 24: 105 (2007) Literature: Aptroot et al., 2007

543. Hypogymnia coilocarpa Vain., Suom. Elain-ja Kasvit. Seuran. Van. Julk. 1: 40 (1921)

Literature: Vainio 1921; Alava 1988 as Parmelia coilocarpa; Wolseley et al., 2002

544. Hypogymnia pseudobitteriana (D.D.Awasthi) D.D.Awasthi, Geophytology 1: $101(1971)$

Literature: Wolseley et al., 2002; Louwhoff et al., 2012

545. Hypogymnia subphysodes (Kremp.) Filson, Vict. Naturalist 87: 325 (1970)

Literature: Vainio 1921 as Parmelia subphysodes; Wolseley and Aguirre-Hudson 1997a; Wolseley et al., 2002

546. Hypogymnia vittata (Ach.) Parrique, Bull. Soc. Linn. Bordeaux 53: 66 (1898)

Literature: Wolseley and Aguirre-Hudson 1997a; Wolseley et al., 2002; Louwhoff et al., 2012

547. Hypotrachyna addita (Hale) Hale, Phytologia 28: 340 (1974)

Literature: Louwhoff et al., 2012

548. Hypotrachyna adjuncta (Hale) Hale, Phytologia 28: 340 (1974)

Literature: Pooprang et al., 1999; Moon et al., 2000b; Wolseley et al., 2002; Louwhoff et al., 2012

549. Hypotrachyna bahiana (Nyl.) Hale, Smithsonian Contr. Bot. 25: 23 (1975)

Literature: Wolseley et al., 2002 
550. Hypotrachyna brevirhiza (Kurok.) Hale, Smithsonian Contr. Bot. 25: 26 (1975) Literature: Buaruang et al., 2009; Louwhoff et al., 2012

551. Hypotrachyna chlorobarbatica Elix \& Pooprang, in Pooprang et al., Mycotaxon 71: 113 (1999)

Literature: Pooprang et al., 1999; Wolseley et al., 2002; Louwhoff et al., 2012

552. Hypotrachyna cirrhata (Fr.) Divakar, A.Crespo, Sipman, Elix \& Lumbsch, Phytotaxa 132: 31 (2013)

Literature: Vainio 1921 as Parmelia vulgaris; Satô 1962 as Parmelia cirrhata; Moon et al., 2000a and Wolseley et al., 2002 as Everniastrum cirrhatum

553. Hypotrachyna coorgiana Patw. \& Prabhu., Bryologist 80: 348 (1977)

Literature: Pooprang et al., 1999; Wolseley et al., 2002; Buaruang et al., 2009

554. Hypotrachyna corneola Kurok. \& K.H.Moon, Bull. Bot. Gard. Toyama 5: 12 (2000)

Literature: Louwhoff et al., 2012

555. Hypotrachyna crenata (Kurok.) Hale, Phytologia28: 341 (1974)

Literature: Wolseley et al., 2002; Louwhoff et al., 2012

556. Hypotrachyna dactylifera (Vain.) Hale, Smithsonian Contr. Bot. 25: 30 (1975) Literature: Moon et al., 2000a; Wolseley et al., 2002

557. Hypotrachyna ducalis (Jatta) Hale, Smithsonian Contr. Bot. 25: 33 (1975) Literature: Boonpragob et al., 1998; Wolseley et al., 2002

558. Hypotrachyna expallida (Kurok.) Divakar, A.Crespo, Sipman, Elix \& Lumbsch, Phytotaxa 132: 33 (2013)

Literature: Hale 1976b as Parmelina expallida; Moon et al., 2000a; Wolseley et al., 2002; Buaruang et al., 2009; Wolseley and Aguirre-Hudson 1995; Louwhoff et al., 2012 as Parmelinopsis expallida

559. Hypotrachyna exsecta (Taylor) Hale, Phytologia 28: 341 (1974)

Literature: Satô 1962 as Parmelia exsecta; Moon et al., 2000a; Louwhoff et al., 2012

560. Hypotrachyna formosana (Zahlbr.) Hale, Smithsonian Contr. Bot. 25: 38 (1975)

Literature: Boonpragob et al., 1998; Wolseley and Aguirre-Hudson 1995

561. Hypotrachyna granulans K.H.Moon, Kurok. \& Kashiw., Bull. Natl. Sci. Mus., Tokyo, B. 26: 135 (2000)

Literature: Moon et al., 2000b; Wolseley et al., 2002; Louwhoff et al., 2012

562. Hypotrachyna heterochroa (Hale \& Kurok.) Elix, Lich. Aust. Exsicc. 4: 82 (1985) Literature: Vainio 1921 as Parmelia bahiana

563. Hypotrachyna horrescens (Taylor) Krog \& Swinscow, Lichenologist 19: 420 (1987)

Literature: Pooprang et al., 1999; Wolseley et al., 2002; Louwhoff et al., 2012 as Parmelinopsis horrescens

564. Hypotrachyna imbricatula (Zahlbr.) Hale, Smithsonian Contr. Bot. 25: 41 (1975)

Literature: Moon et al., 2000a; Wolseley et al., 2002; Louwhoff et al., 2012 
565. Hypotrachyna immaculata (Kurok.) Hale, Smithsonian Contr. Bot. 25: 41 (1975)

Literature: Pooprang et al., 1999; Wolseley et al., 2002; Louwhoff et al., 2012

566. Hypotrachyna infirma (Kurok.) Hale, Phytologia 28: 341 (1974)

Literature: Moon et al., 2000a; Wolseley et al., 2002

567. Hypotrachyna masonhalei Patw. \& Prabhu, Bryologist 80: 348 (1977)

Literature: Pooprang et al., 1999; Wolseley et al., 2002; Louwhoff et al., 2012

568. Hypotrachyna massartii (Hue) Hale, Phytologia 28: 341 (1974)

Literature: Moon et al., 2000a; Wolseley et al., 2002

569. Hypotrachyna microlobulata (D.D.Awasthi) Divakar, A.Crespo, Sipman, Elix \& Lumbsch, Phytotaxa 132: 34 (2013)

Literature: Pooprang et al., 1999; Wolseley et al., 2002; Buaruang et al., 2009; Louwhoff et al., 2012 as Parmelinopsis microlobulata

570. Hypotrachyna minarum (Vain.) Krog \& Swinscow, Lichenologist 19: 420 (1987) Literature: Pooprang et al., 1999; Wolseley et al., 2002; Louwhoff et al., 2012 as Parmelinopsis minarum

571. Hypotrachyna nepalensis (Taylor) Divakar, A.Crespo, Sipman, Elix \& Lumbsch, Phytotaxa 132: 32 (2013)

Literature: Satô 1962 and Vainio 1921 as Parmelia americana; Wolseley and Aguirre-Hudson 1995; 1997a; Moon et al., 2000a; Wolseley et al., 2002 and Louwhoff et al., 2012 as Everniastrum nepalense

572. Hypotrachyna orientalis (Hale) Hale, Phytologia 28: 341 (1974)

Literature: Wolseley and Aguirre-Hudson 1995; Boonpragob et al., 1998; Moon et al., 2000a; Wolseley et al., 2002; Louwhoff et al., 2012

573. Hypotrachyna osseoalba (Vain.) Y.S.Park \& Hale, Taxon 38: 88 (1989)

Literature: Alava 1988 as Parmelia osseoalba; Vainio 1921; Moon et al., 2000a; Wolseley et al., 2002; Buaruang et al., 2009; Louwhoff et al., 2012

574. Hypotrachyna physcioides (Nyl.) Hale, Smithsonian Contr. Bot. 25: 54 (1975) Literature: Pooprang et al., 1999; Moon et al., 2000a; Wolseley et al., 2002; Louwhoff et al., 2012

575. Hypotrachyna ramkhamhaengiana Elix \& Pooprang, in Pooprang et al., Mycotaxon 71: 113 (1999)

Literature: Pooprang et al., 1999; Wolseley et al., 2002; Louwhoff et al., 2012

576. Hypotrachyna scabrida (Elix \& Pooprang) Divakar, A.Crespo, Sipman, Elix \& Lumbsch, Phytotaxa 132: 32 (2013)

Literature: Pooprang et al., 1999; Wolseley et al., 2002; Louwhoff et al., 2012 as Everniastrum scabridum

577. Hypotrachyna scytodes (Kurok.) Hale, Phytologia 28: 341 (1974)

Literature: Moon et al., 2000a; Wolseley et al., 2002

578. Hypotrachyna sorocheila (Vain.) Divakar, A.Crespo, Sipman, Elix \& Lumbsch, Phytotaxa 132: 32 (2013)

Literature: Satô 1962 as Parmelia sorocheila; Wolseley et al., 2002 as Everniastrum sorocheilum 
579. Hypotrachyna vexans (Zahlbr. ex W.L.Culb. \& C.F.Culb.) Divakar, A.Crespo, Sipman, Elix \& Lumbsch, Phytotaxa 132: 33 (2013)

Literature: Wolseley and Aguirre-Hudson 1995; 1997a; Moon et al., 2000a; Wolseley et al., 2002; Buaruang et al., 2009; Louwhoff et al., 2012 as Everniastrum vexans

580. Imshaugia placorodia (Ach.) S.L.F.Mey., Mycologia 77: 338 (1985)

Literature: Wolseley et al., 2002

581. Ionaspis tropica Aptroot, in Aptroot, Diederich, Sérusiaux \& Sipman, Biblioth. Lichenol. 64: 73 (1997)

Literature: Boonpragob et al., 1998; Wolseley et al., 2002

582. Julella lactea (A.Massal.) M.E.Barr, Sydowia 38: 13 (1986) [1985]

Literature: Aptroot et al., 2007

583. Kalbionora palaeotropica Sodamuk, Leavitt \& Lumbsch, MycoKeys 22: 17 (2017)

Literature: Sodamuk et al., 2017

584. Lasioloma phycophilum (Vain.) R.Sant., Symb. Bot. Upsal. 12: 550 (1952)

Literature: Papong et al., 2007

585. Lecanactis flavisedella (Nyl.) Tehler, Willdenowia 22: 206 (1992)

Literature: Wolseley and Aguirre-Hudson 1997a; Wolseley et al., 2002; Aptroot et al., 2007

586. Lecanactis platygraphoides (Müll.Arg.) Zahlbr., Cat. Lich. Univ. 2: 541 (1923) [1924]

Literature: Aptroot et al., 2007

587. Lecanactis subfarinosa (C.Knight) Hellb., Bih. Kongl.. Svenska Vetensk.-Akad. Handl. 21: 120 (1896)

Literature: Aptroot et al., 2007

588. Lecanographa atropunctata Sparrius, Saipunkaew \& Wolslely, Lichenologist 38: 33 (2006)

Literature: Sparrius et al., 2006

589. Lecanora achroa Nyl., in Crombie, J. Linn. Soc., Bot. 14: 26 (1876)

Literature: Vainio 1909 as Lecanora monodorae and Lecanora subfusa v. chlarona; Wolseley et al., 2002 as Lecanora pulicaris and Lecanora achroella; Aptroot et al., 2007; Papong and Lumbsch 2011

590. Lecanora argentata (Ach.) Röhl, Deutschl. Fl., Abth. 2: 82 (1813)

Literature: Paulson 1930; Wolseley and Aguirre-Hudson 1997b; Wolseley et al., 1994 and Wolseley et al., 2002 as Lecanora subfusca; Papong and Lumbsch 2011

591. Lecanora arthothelinella Lumbsch, J. Hattori Bot. Lab.77: 71 (1994)

Literature: Papong et al., 2011a; Papong and Lumbsch 2011

592. Lecanora austrotropica Lumbsch, J. Hattori Bot. Lab. 77: 72 (1994)

Literature: Papong et al., 2011a; Papong and Lumbsch 2011

593. Lecanora caesiorubella Ach., Lichenogr. Universalis: 366 (1810)

Literature: Aptroot et al., 2007; Papong and Lumbsch 2011 
594. Lecanora coronulans Nyl., Flora 59: 510 (1876)

Literature: Papong and Lumbsch 2011

595. Lecanora ecoronata Vain., Bolet. Socied. Broteriana 6: 146, 1929-30

Literature: Aptroot et al., 2007; Papong and Lumbsch 2011

596. Lecanora farinacea Fée, Essai Crypt. Écorc. (Paris): 117 (1825) [1824]

Literature: Papong and Lumbsch 2011

597. Lecanora flavoviridis Kremp., Flora 56: 470 (1873)

Literature: Aptroot et al., 2007; Papong and Lumbsch 2011

598. Lecanora fumigata Vain., Ann. Bot. Soc. Zool.-Bot. Fenn. "Vanamo" 1: 42 (1921)

Literature: Vainio 1921; Wolseley et al., 2002

599. Lecanora helva Stizenb., Ber. Tät. St. Gall. Naturw. Ges.: 218 (1890) [188889]

Literature: Aptroot et al., 2007; Papong and Lumbsch 2011; Schumm and Aptroot 2012

600. Lecanora interjecta Müll.Arg., Nuovo Giorn. Bot. Ital. 23: 390 (1891)

Literature: Papong and Lumbsch 2011

601. Lecanora kansriae Papong \& Lumbsch, Lichenologist 43: 308 (2011)

Literature: Papong and Lumbsch 2011

602. Lecanora leproplaca Zahlbr., in Magnusson \& Zahlbruckner, Ark. Bot. 31: 64 (1944)

Literature: Papong and Lumbsch 2011

603. Lecanora leprosa Fée, Essai Crypt. Écorc. (Paris): 118 (1825) [1824]

Literature: Vainio 1909; 1921; Wolseley et al., 2002 as Lecanora cinereocarnea;

Papong and Lumbsch 2011; Schumm and Aptroot 2012

604. Lecanora melaleuca (Müll.Arg.) C.W.Dodge, Beih. Nova Hedwigia 38: 22 (1971)

Literature: Papong and Lumbsch 2011

605. Lecanora phaeocardia Vain., Ann. Bot. Soc. Zool.-Bot. Fenn. "Vanamo" 1: 41 (1923)

Literature: Vainio 1921 as Lecanora phaeocardia var. subcrenulans and Lecanora phaeocardia var. lividofuscescens; Wolseley et al., 2002; Aptroot et al., 2007; Papong and Lumbsch 2011

606. Lecanora plumosa Müll.Arg., Flora 65: 484 (1882)

Literature: Papong and Lumbsch 2011

607. Lecanora pseudargentata Lumbsch, J. Hattori Bot. Lab.77: 127 (1994)

Literature: Papong and Lumbsch 2011

608. Lecanora pseudistera Nyl., Flora 55: 354 (1872)

Literature: Aptroot et al., 2007; Papong and Lumbsch 2011

609. Lecanora subimmergens Vain., Bot. Mag. (Tokyo) 35: 51 (1921)

Literature: Papong et al., 2011a; Papong and Lumbsch 2011

610. Lecanora subimmersa Müll.Arg., Nova Hedwigia 32: 124 (1893)

Literature: Aptroot et al., 2007; Papong and Lumbsch 2011 
611. Lecanora toroyensis Zahlbr., Feddes Repert. 33: 52 (1933)

Literature: Papong and Lumbsch 2011

612. Lecanora tropica Zahlbr., Cat. Lich. Univ. 5: 589 (1928)

Literature: Vainio 1909; Wolseley et al., 2002 as Lecanora subgranulata; Aptroot et al., 2007 as Lecanora casuarinophila; Papong and Lumbsch 2011

613. Lecanora ulrikii Papong \& Lumbsch, Lichenologist 43: 48 (2010)

Literature: Papong et al., 2011a; Papong and Lumbsch 2011

614. Lecanora vainioi Vänskä, Ann. Bot. Fenn. 23: 123 (1986)

Literature: Aptroot et al., 2007; Papong and Lumbsch 2011

615. Lecidopyrenopsis corticola Vain., Hedwigia 46: 172 (1907)

Literature: Vainio 1909; Alava 1988; Wolseley et al., 2002

616. Leioderma sorediatum D.J.Galloway \& P.M.Jørg., Lichenologist 19: 390 (1987)

Literature: Jørgensen and Wolseley 2009

617. Leiorreuma hypomelaenoides Poengs. \& Kalb, Phytotaxa 189: 255-267 (2014)

Literature: Poengsungnoen et al., 2014b

618. Leiorreuma sericeum (Eschw.) Staiger, Biblioth. Lichenol. 85: 305 (2002)

Literature: Vainio 1909 as Graphina sericea; Wolseley et al., 2002 as Phaeographis sericea

619. Lepidocollema brisbanense (C. Knight) P.M. Jørg., in Ekman, Wedin, Lindblom \& Jørgensen, Lichenologist 46: 650 (2014)

Literature: Wolseley and Aguirre-Hudson 1995, 1997a, 1997b; Boonpragob et al., 1998; Wolseley et al., 2002 as Parmeliella brisbanensis; Rangsiruji et al., 2016

620. Lepidocollema marianum (Fr.) P.M. Jørg., in Ekman, Wedin, Lindblom \& Jørgensen, Lichenologist 46: 651 (2014)

Literature: Rangsiruji et al., 2016

621. Lepidocollema papillatum (P.M. Jørg.) P.M. Jørg., in Ekman, Wedin, Lindblom \& Jørgensen, Lichenologist 46: 651 (2014)

Literature: Wolseley et al., 2002 as Parmeliella papillata

622. Lepidocollema wainioi (Zahlbr.) P.M. Jørg., in Ekman, Wedin, Lindblom \& Jørgensen, Lichenologist 46: 651 (2014)

Literature: Rangsiruji et al., 2016

623. Lepra amara (Ach.) Hafellner in Hafellner \& Türk, Stapfia 104: 171 (2016)

Literature: Wolseley and Aguirre-Hudson 1997a; Aptroot et al., 2007 as Pertusaria amara

624. Lepra buloloensis (A.W.Archer, Elix \& Streimann) Schmitt \& Lumbsch Literature: Aptroot et al., 2007 as Pertusaria bulolensis

625. Lepra patellifera (A.W.Archer) Schmitt \& Lumbsch Literature: Aptroot et al., 2007 as Pertusaria patellifera

626. Lepra subventosa (Malme) Schmitt \& Lumbsch Literature: Aptroot et al., 2007 as Pertusaria subventosa

627. Lepraria arbuscula (Nyl.) Lendemer \& B.P. Hodk., Mycologia 105: 1005 (2013) Literature: Wolseley and Aguirre-Hudson 1995, 1997a; Boonpragob et al., 1998; Wolseley et al., 2002 as Leprocaulon arbuscula 
628. Lepraria aurescens Orange \& Wolseley, Lichenologist 37: 247 (2005)

Literature: Orange and Wolseley 2005

629. Lepraria pulchra Orange \& Wolseley, Lichenologist 37: 249 (2005)

Literature: Orange and Wolseley 2005

630. Leprocollema novacaledonicum A.L.Sm., J. Linn. Soc., Bot. 46: 78 (1922)

Literature: Aptroot et al., 2007

631. Leptogium asiaticum P.M.Jørg., Herzogia 2: 466 (1973)

Literature: Wolseley and Aguirre-Hudson 1997a; Wolseley et al., 2002

632. Leptogium austroamericanum (Malme) C.W.Dodge, Ann. Missouri Bot. Gard. 20: 419 (1933)

Literature: Aptroot et al., 2007

633. Leptogium azureum (Ach.) Mont., in Webb \& Berthelot, Hist. Nat. Iles Canaries (Phytogr.) 3: 129 (1840)

Literature: Boonpragob et al., 1998; Wolseley et al., 2002

634. Leptogium burgessii (L.) Mont., in Webb \& Berthelot, Hist. Nat. Iles Canaries (Phytogr.) 3: 130 (1840)

Literature: Wolseley et al., 2002

635. Leptogium caesium (Ach.) Vain., Acta Soc. Fauna Fl. Fenn. 7: 225 (1890)

Literature: Vainio 1909; Wolseley et al., 2002

636. Leptogium chloromelum (Ach.) Nyl., Mém. Soc. Sci. Nat. Cherbourg 5: 333 (1857)

Literature: Paulson 1930; Wolseley et al., 2002

637. Leptogium cochleatum (Dicks.) P.M.Jørg. \& P.James, Lichenologist 15: 113 (1983)

Literature: Paulson 1930 as Leptogium tremelloides; Wolseley et al., 2002; Rangsiruji et al., 2016

638. Leptogium coralloideum (Meyen \& Flot.) Vain., Ann. Acad. Sci. Fenn., Ser. A, 6: 110 (1915) Literature: Vainio 1921 as Parmelia coralloidea; Wolseley et al., 2002

639. Leptogium corticola (Taylor) Tuck. in Lea, Cat. Pl. Cincinnati: 47 (1849) Literature: Aptroot et al., 2007

640. Leptogium cyanescens (Rabenh.) Körb., Syst. Lich. Germ.: 420 (1855)

Literature: Wolseley and Aguirre-Hudson 1997a; 1997b; Boonpragob et al., 1998; Wolseley et al., 2002; Papong et al., 2007; Rangsiruji et al., 2016

641. Leptogium denticulatum Nyl., Ann. Sci. Nat., Bot. 5: 302 (1867)

Literature: Wolseley and Aguirre-Hudson 1997a; 1997b; Boonpragob et al., 1998; Wolseley et al., 2002

642. Leptogium javanicum (Mont. \& Bosch) Mont., Syll. Gen. Sp. Crypt.: 379 (1856)

Literature: Wolseley and Aguirre-Hudson 1997a; Wolseley et al., 2002

643. Leptogium marginellum (Sw.) Gray, Nat. Arr. Brit. Pl. 1: 401 (1821)

Literature: Mongkolsuk et al., 1996; Wolseley and Aguirre-Hudson 1997a;

Wolseley et al., 2002 
644. Leptogium moluccanum (Pers.) Vain., Acta Soc. Fauna Fl. Fenn. 7: 223 (1890) Literature: Satô 1962; Vainio 1921; Wolseley et al., 2002

645. Leptogium pedicellatum P.M.Jørg., Herzogia 3: 448 (1975) [1973-4]

Literature: Aptroot et al., 2007

646. Leptogium phyllocarpum (Pers.) Mont., Ann. Sci. Nat., Bot. 10: 134 (1848) Literature: Aptroot et al., 2007

647. Leptogium resupinans Nyl., Ann. Sci. Nat., Bot. 15: 368 (1861)

Literature: Wolseley et al., 2002

648. Leptogium trichophorum Müll.Arg. Flora 72: 505 (1889)

Literature: Vainio 1921; Wolseley et al., 2002

649. Letrouitia corallina (Müll.Arg.) Hafellner, Nova Hedwigia 35: 669 (1983) [1981] Literature: Aptroot et al., 2007

650. Letrouitia domingensis (Pers.) Hafellner \& Bellem., Nova Hedwigia 35: 281 (1982) [1981]

Literature: Wolseley and Aguirre-Hudson 1997b; Boonpragob et al., 1998; Wolseley et al., 1994; 2002; Schumm and Aptroot 2012

651. Letrouitia flavocrocea (Nyl.) Hafellner \& Bellem., Nova Hedwigia 35: 281 (1982) [1981]

Literature: Wolseley et al., 1994; 2002

652. Letrouitia leprolyta (Nyl.) Hafellner, Nova Hedwigia 35: 693 (1983) [1981]

Literature: Wolseley and Aguirre-Hudson 1997a; 1997b; Wolseley et al., 2002

653. Letrouitia subvulpina (Nyl.) Hafellner, Nova Hedwigia 35: 705 (1983) [1981] Literature: Wolseley and Aguirre-Hudson 1997a; 1997b; Boonpragob et al., 1998; Wolseley et al., 1994; 2002

654. Letrouitia transgressa (Malme) Hafellner \& Bellem., Nova Hedwigia 35: 710 (1983) Literature: Wolseley et al., 1994; 2002

655. Letrouitia vulpina (Tuck.) Hafellner \& Bellem., Nova Hedwigia 35: 281 (1982) [1981]

Literature: Wolseley and Aguirre-Hudson 1997b; Wolseley et al., 1994; 2002

656. Leucodecton anamalaiense (Patw. \& C.R.Kulk.) Rivas Plata \& Lucking, in Rivas Plata et al., Lichenologist 42: 184 (2010)

Literature: Papong et al., 2014

657. Leucodecton compunctellum (Nyl.) Frisch, Biblioth. Lichenol. 92: 155 (2006) Literature: Schumm and Aptroot 2012; Parnmen et al., 2012

658. Leucodecton compunctum (Ach.) A.Massal., Atti Ist. Veneto Sci. Lett. Arti 5: 325 (1860) [1859-1860]

Literature: Wolseley et al., 2002 as Myriotrema compunctum

659. Leucodecton confusum Papong, Lumbsch, Lucking in Papong et al., Phytotaxa 189: 233 (2014)

Literature: Papong et al., 2014

660. Leucodecton fissurinum (Hale) Frisch, Biblioth. Lichenol. 92: 156 (2006)

Literature: Boonpragob et al., 1998 and Wolseley et al., 2002 as Myriotrema decorticatum and Myriotrema fissurinum 
661. Leucodecton glaucescens (Nyl.) Frisch, Biblioth. Lichenol. 92: 162 (2006)

Literature: Wolseley et al., 2002 as Myriotrema glaucescens

662. Leucodecton phaeosporum (Nyl.) Rivas Plata \& Lücking, in Rivas Plata et al., Lichenologist 42: 184 (2010)

Literature: Paulson 1930 as Leptotrema phaeosporum; Wolseley et al., 2002 as Myriotrema phaeosporum

663. Leucodermia borphyllidiata Kalb \& Meesim, Phytotaxa 235:1-66 (2015)

Literature: Mongkolsuk et al., 2015

664. Leucodermia boryi (Fée) Kalb, Phytotaxa 235:1-66 (2015)

Literature: Satô 1962 as Anaptychia neoleucomelaena; Mongkolsuk et al., 2015

665. Leucodermia leucomelos (L.) Kalb, Phytotaxa 235:1-66 (2015)

Literature: Vainio 1921 as Anaptychia leucomelaena; Wolseley and AguirreHudson 1995; 1997a; Wolseley et al., 2002; Meesim and Mongkolsuk 2010a; Mongkolsuk and Meesim 2012 as Heterodermia leucomelos

666. Lithothelium obtectum (Müll.Arg.) Aptroot, Biblioth. Lichenol. 44: 62 (1991) Literature: Aptroot et al., 2007

667. Lithothelium quadrisporum Aptroot, Lichenologist 38: 543 (2006)

Literature: Aptroot 2006

668. Lobaria japonica (Zahlbr.) Asahina, Jap. J. Bot. 9: 450 (1933)

Literature: Aptroot et al., 2007

669. Lobaria pseudopulmonaria Gyeln., Acta Fauna. Fl. Universali, ser 2, Bot.: 6 (1933)

Literature: Yoshimura 1978; Wolseley et al., 2002

670. Lobaria retigera (Bory) Trevis., Philipp. J. Sci. 8: 129 (1913)

Literature: Satô 1962; Wolseley and Aguirre-Hudson 1995; 1997a; Wolseley et al., 2002

671. Lobaria retigera var. subisidiosa (Asahina) Yoshim., Misc. Bryol. Lichenol. 6: 135 (1974)

Literature: Yoshimura 1978

672. Lobariella isidiosa Moncada \& Lücking, Lichenologist 45: 203-263 (2013)

Literature: Satô 1962; Wolseley et al., 2002 as Lobaria isidiosa

673. Lopezaria isidiza (Makhija \& Nagarkar) Aptroot \& Sipman, in Aptroot et al., Fungal Diversity 24: 110 (2007)

Literature: Aptroot et al., 2007

674. Lopezaria versicolor (Flot.) Kalb \& Hafellner, in Kalb, Lichenes Neotropici, Fascicle 11: 464 (1990)

Literature: Aptroot et al., 2007

675. Loxospora lecanoriformis Lumbsch, A.W.Archer \& Elix, Lichenologist 39: 514 (2007)

Literature: Papong et al., 2009a

676. Malmidea aurigera (Fée) Kalb, Rivas Plata \& Lumbsch, in Kalb et al., Biblioth. Lichenol. 106: 153 (2011)

Literature: Aptroot et al., 2007; Kalb et al., 2011 
677. Malmidea bakeri (Vain.) Kalb, Rivas Plata \& Lumbsch, in Kalb et al., Biblioth. Lichenol.106: 154 (2011)

Literature: Kalb et al., 2011; Schumm and Aptroot 2012

678. Malmidea ceylanica (Zahlbr.) Kalb, Rivas Plata \& Lumbsch, in Kalb et al., Biblioth. Lichenol. 106: 164 (2011)

Literature: Schumm and Aptroot 2012

679. Malmidea chrysostigma (Vain.) Kalb, Rivas Plata \& Lumbsch, in Kalb et al., Biblioth. Lichenol. 106: 155 (2011)

Literature: Kalb et al., 2011

680. Malmidea coralliformis Kalb, in Kalb et al., Biblioth. Lichenol. 106: 157 (2011) Literature: Kalb et al., 2011

681. Malmidea duplomarginata (Papong \& Kalb) Kalb \& Papong, in Kalb et al., Biblioth. Lichenol. 106: 165 (2011)

Literature: Kalb et al., 2009b as Malcolmiella duplomarginata

682. Malmidea eeunae Kalb, in Kalb et al., Biblioth. Lichenol. 106: 158 (2011) Literature: Kalb et al., 2011

683. Malmidea granifera (Ach.) Kalb, Rivas Plata \& Lumbsch, in Kalb et al., Biblioth. Lichenol. 106: 165 (2011)

Literature: Aptroot et al., 2007 as Malcolmiella granifera

684. Malmidea inflata Kalb, in Kalb et al., Biblioth. Lichenol. 106: 159 (2011)

Literature: Kalb et al., 2011

685. Malmidea perplexa Kalb, in Kalb et al., Biblioth. Lichenol. 106: 160 (2011) Literature: Kalb et al., 2011

686. Malmidea piae (Kalb) Kalb Rivas Plata \& Lumbsch, in Kalb et al., Biblioth. Lichenol. 106: 161 (2011)

Literature: Kalb et al., 2009b as Malcolmiella piae

687. Malmidea subaurigera (Vain.) Kalb, Rivas Plata \& Lumbsch, in Kalb et al., Biblioth. Lichenol.106: 161 (2011)

Literature: Kalb et al., 2011

688. Malmidea subgranifera (Kalb \& Elix) Kalb \& Elix, in Kalb et al., Biblioth. Lichenol.106: 166 (2011)

Literature: Schumm and Aptroot 2012

689. Malmidea tratiana Kalb \& Mongkolsuk, in Kalb et al., Phytotaxa 42: 44 (2012) Literature: Kalb et al., 2012; Schumm and Aptroot 2012

690. Malmidea variabilis Kalb, in Kalb et al., Biblioth. Lichenol.106: 162 (2011) Literature: Kalb et al., 2011

691. Malmidea vinosa (Eschw.) Kalb, Rivas Plata \& Lumbsch, in Kalb et al., Biblioth. Lichenol. 106: 166 (2011)

Literature: Aptroot et al., 2007 as Malcolmiella vinosa

692. Marcelaria benguelensis (Müll.Arg.) Aptroot, Nelsen \& Parnmen, Glalia 5: 4 (2013) Literature: Boonpragob et al., 1998; Wolseley and Aguirre-Hudson 1997b; Wolseley et al., 2002 as Laurera benguelensis; Schumm and Aptroot 2012 as Laurera subbenguelensis; Aptroot and Lücking 2016 
693. Maronea constans (Nyl.) Hepp, Flechten Eur.: 771 (1860)

Literature: Wolseley and Aguirre-Hudson 1997a; Wolseley et al., 2002

694. Maronina corallifera (Kantvilas \& Papong) Divakar, Crespo \& Lumbsch, Fungal Diversity. https://doi.org/10.1007/s13225-017-0379-z (2017)

Literature: Papong et al., $2011 \mathrm{~b}$ as Maronina orientalis var. corallifera; Kantvilas et al., 2010 as Protoparmelia corallifera

695. Maronina isidiata (Diederich, Aptroot \& Sérus.) Divakar, Crespo \& Lumbsch, Fungal Diversity. https://doi.org/10.1007/s13225-017-0379-z (2017)

Literature: Aptroot et al., 2007 as Protoparmelia isidiata

696. Maronina orientalis Kantvilas \& Papong, Lichenologist 42: 557 (2010)

Literature: Papong et al., 2011b; Kantvilas et al., 2010 as Protoparmelia orientalis

697. Maronina pulchra (Diederich, Aptroot \& Sérus.) Divakar, Crespo \& Lumbsch, Fungal Diversity. https://doi.org/10.1007/s13225-017-0379-z (2017)

Literature: Aptroot et al., 2007 as Protoparmelia pulchra

698. Mazosia dispersa (J.Hedrick) R.Sant., Symb. Bot. Upsal. 12: 120 (1952)

Literature: Papong et al., 2007

699. Mazosia melanophthalma (Müll.Arg.) R.Sant., Symb. Bot. Upsal. 12: 117 (1952)

Literature: Boonpragob et al., 1998; Wolseley et al., 2002

700. Mazosia paupercula (Müll.Arg.) R.Sant., Symb. Bot. Upsal. 12: 128 (1952)

Literature: Papong et al., 2007

701. Mazosia phyllosema (Nyl.) Zahlbr., Cat. Lich. Univ. 2: 503 (1923)

Literature: Aptroot et al., 2007; Papong et al., 2007

702. Mazosia pseudobambusae Kalb \& Vězda, Folia Geobot. Phytotax. 23: 207 (1988)

Literature: Papong et al., 2007

703. Mazosia rotula (Mont.) A.Massal., Neagen. Lich.: 9 (1854)

Literature: Papong et al., 2007

704. Megalaria laureri (Hepp ex Th.Fr.) Hafellner, The lichens of Italy. An annotated catalogue: 429 (1993)

Literature: Aptroot et al., 2007

705. Megalospora bicolorata (Vain.) Zahlbr., Cat. Lich. Univ. 4: 87 (1926) [1927]

Literature: Vainio 1921 as Catillaria bicolorata; Wolseley et al., 2002

706. Megalospora coccodes (Bél.) Sipman, Biblioth. Lichenol. 18: 132 (1983)

Literature: Aptroot et al., 2007

707. Megalospora sulphurata Meyen, in Meyen \& Flot., Nova Acta Acad. Caes. Leop.-Carol. German. Nat. Cur. 19: 228 (1843)

Literature: Wolseley and Aguirre-Hudson 1997b; Wolseley et al., 2002

708. Megalospora tuberculosa (Fée) Sipman, Biblioth. Lichenol. 18: 156 (1983)

Literature: Boonpragob et al., 1998; Wolseley et al., 2002

709. Megalotremis biocellata Aptroot, Biblioth. Lichenol. 44: 125 (1991)

Literature: Aptroot et al., 2007; 2008 
710. Megalotremis pustulata Aptroot, in Aptroot et al., Biblioth. Lichenol. 64: 103 (1997)

Literature: Aptroot et al., 2007

711. Melanotrema lirelliforme (Tuck.) Frisch, in Frisch \& Kalb, Biblioth. Lichenol. 92: 390 (2006)

Literature: Aptroot et al., 2007 as Ocellularia lirelliformis

712. Melanotrema meiospermum (Nyl.) Frisch, in Frisch \& Kalb, Biblioth. Lichenol. 92: 394 (2006)

Literature: Wolseley et al., 2002 as Ocellularia meiosperma; Sutjaritturakan and Kalb 2015

713. Melanotrema melanophthalmum (Homchant. \& Coppins) Papong \& Lumbsch, in Papong et al., Lichenologist 42: 135 (2010)

Literature: Yoshimura 1978; Homchantara and Coppins 2002; Wolseley et al., 2002; Papong et al., 2010 as Ocellularia melanophthalma

714. Melanotrema platystomum (Mont.) Frisch, in Frisch \& Kalb, Biblioth. Lichenol. 92: 397 (2006)

Literature: Boonpragob et al., 1998; Wolseley et al., 2002 as Ocellularia concolor; Sutjaritturakan and Kalb 2015

715. Menegazzia terebrata (Hoffm.) A.Massal., Neagen. Lich.: 1 (1854)

Literature: Wolseley and Aguirre-Hudson 1995; 1997a; Wolseley et al., 2002;

Louwhoff et al., 2012

716. Microtheliopsis uleana Müll.Arg., Flora 73: 195 (1890)

Literature: Boonpragob et al., 1998; Wolseley et al., 2002

717. Milospium planorbis Aptroot \& Sipman, J. Hattori Bot. Lab. 91: 331 (2001) Literature: Aptroot et al., 2007

718. Mycoporum compositum (A.Massal.) R.C.Harris, in Egan, Bryologist 90: 164 (1987)

Literature: Aptroot et al., 2007

719. Myelochroa aurulenta (Tuck.) Elix \& Hale, Mycotaxon 29: 240 (1987)

Literature: Moon et al., 2000a; Wolseley et al., 2002; Louwhoff et al., 2012

720. Myelochroa entotheiochroa (Hue) Elix \& Hale, Mycotaxon 29: 240 (1987)

Literature: Moon et al., 2000a; Wolseley et al., 2002; Louwhoff et al., 2012

721. Myelochroa irrugans (Nyl.) Elix \& Hale, Mycotaxon 29: 241 (1987)

Literature: Moon et al., 2000a; Wolseley et al., 2002; Louwhoff et al., 2012

722. Myelochroa perisidians (Nyl.) Elix \& Hale, Mycotaxon 29: 241 (1987)

Literature: Hale 1976b as Parmelina perisidians; Moon et al., 2000a; Wolseley et al., 2002

723. Myelochroa siamea Kurok., J. Jap. Bot. 73: 13 (1998)

Literature: Kurokawa 1998; Moon et al., 2000a; Wolseley et al., 2002; Louwhoff et al., 2012

724. Myelochroa subaurulenta (Nyl.) Elix \& Hale, Mycotaxon 29: 241 (1987)

Literature: Louwhoff et al., 2012 
725. Myelochroa xantholepis (Mont. \& Bosch) Elix \& Hale, Mycotaxon 29: 241 (1987)

Literature: Hale 1976b as Parmelina xantholepis; Moon et al., 2000a; Wolseley et al., 2002; Louwhoff et al., 2012

726. Myeloconis erumpens P.M.McCarthy \& Elix, Lichenologist 28: 406 (1996)

Literature: Boonpragob et al., 1998; Wolseley et al., 2002; Aptroot et al., 2007

727. Myriotrema clandestinum (Fée) Hale, Mycotaxon 11: 133 (1980)

Literature: Boonpragob et al., 1998; Wolseley et al., 2002 as Myriotrema terebratulum

728. Myriotrema fluorescens Hale, Bull. Brit. Mus. (Nat. Hist.), Bot. 8: 281 (1981)

Literature: Aptroot et al., 2007

729. Myriotrema microporum (Mont.) Hale, Mycotaxon 11: 134 (1980).

Literature: Boonpragob et al., 1998; Wolseley et al., 2002

730. Myriotrema minutum (Hale) Hale, Mycotaxon 11: 134 (1980)

Literature: Wolseley et al., 2002

731. Myriotrema olivaceum Fée, Essai Crypt. Écorc. (Paris): 92 (1825) [1824]

Literature: Kraichak et al. 2014; Wolseley et al., 2002

732. Myriotrema polytretum Hale, Bull. Brit. Mus. (Nat. Hist.), Bot. 8: 291 (1981)

Literature: Wolseley et al., 2002

733. Myriotrema rugiferum (Harm.) Hale, Mycotaxon 11: 135 (1980)

Literature: Wolseley et al., 2002

734. Myriotrema subconforme (Nyl.) Hale, Mycotaxon 11: 135 (1980)

Literature: Wolseley et al., 2002

735. Myriotrema viride Nagarkar \& Hale, Mycotaxon 35: 440 (1989)

Literature: Wolseley et al., 2002

736. Nadvornikia expallescens (Nyl.) I. Medeiros, Lücking \& Lumbsch, Fieldiana 9: 18 (2017)

Literature: Boonpragob et al., 1998 and Wolseley et al., 2002 as Thelotrema expallescens

737. Nadvornikia hawaiensis (Tuck.) Tibell, Beih. Nova Hedwigia 79: 672 (1984)

Literature: Aptroot et al., 2007

738. Nephroma helveticum Ach., Lichenogr. Universalis: 523 (1810)

Literature: Wolseley and Aguirre-Hudson 1995 and 1997a as Nephroma tropicum; Wolseley et al., 2002

739. Nephromopsis pallescens (Taylor) Kurok., Mem. Natl. Sci. Mus. (Tokyo) 13: 140 (1980)

Literature: Vainio 1921 as Cetraria wallichiana; Wolseley and Aguirre-Hudson 1995; 1997a; Wolseley et al., 2002 as Cetrariopsis wallichiana; Louwhoff et al., 2012

740. Nephromopsis stracheyi (C.Bab.) Müll.Arg., Flora 74: 374 (1891)

Literature: Wolseley and Aguirre-Hudson 1995 as Nephromopsis ornata; Wolseley et al., 2002 
741. Nephromopsis thailandica (Elix \& M.J. Lai) Divakar, Crespo \& Lumbsch, Fungal Diversity. https://doi.org/10.1007/s13225-017-0379-z (2017)

Literature: Lai and Elix 2002; Louwhoff et al., 2012 as Cetreliopsis thailandica

742. Nigrovothelium tropicum (Ach.) Lücking, M.P. Nelsen \& Aptroot, in Lücking et al., Lichenologist 48: 757 (2016)

Literature: Boonpragob et al., 1998; Wolseley et al., 2002; Schumm and Aptroot 2012 as Trypethelium tropicum; Aptroot and Lücking 2016

743. Nitidochapsa leprieurii (Mont.) Parnmen, Lücking \& Lumbsch, Bryologist 116: 127-133 (2013)

Literature: Wolseley et al., 2002 as Thelotrema leprieurii; Kraichak et al. 2014; Lumbsch et al., 2014; Poengsungnoen et al., 2014b; Lücking et al., 2015

744. Nitidochapsa siamensis Poengs., Lücking \& Lumbsch, Phytotaxa 189: 255-267 (2014)

Literature: Poengsungnoen et al., 2014b

745. Normandina pulchella (Borrer) Nyl., Ann. Sci. Nat., Bot. 15: 382 (1861)

Literature: Boonpragob et al., 1998; Wolseley and Aguirre-Hudson 1995; 1997a; Wolseley et al., 2002

746. Ocellularia albocincta (Hale) Divakar \& Mangold, in Papong et al., Lichenologist 42: 135 (2010)

Literature: Boonpragob et al., 1998; Wolseley et al., 2002 as Myriotrema albocinctum; Kraichak et al., 2014

747. Ocellularia albomaculata Hale, Bull. Brit. Mus. (Nat. Hist.), Bot. 8: 300 (1981) Literature: Wolseley et al., 2002

748. Ocellularia allosporoides (Nyl.) Patw. \& C.R.Kulk., Kavaka 5: 5 (1977)

Literature: Boonpragob et al., 1998; Wolseley et al., 2002 as Ocellularia groenhartii; Parnmen et al., 2012; Kraichak et al., 2014; Lumbsch et al., 2014; Poengsungnoen et al., 2014a Lücking et al., 2015

749. Ocellularia arecae (Vain.) Hale, Mycotaxon 11: 136 (1980)

Literature: Vainio 1909 as Thelotrema arecae; Wolseley et al., 2002

750. Ocellularia ascidioidea Hale, Bull. Brit. Mus. (Nat. Hist.), Bot. 8: 300 (1981)

Literature: Boonpragob et al., 1998; Wolseley et al., 2002

751. Ocellularia bahiana (Ach.) Frisch, in Frisch \& Kalb, Biblioth. Lichenol. 92: 503 (2006)

Literature: Wolseley et al., 2002 as Myriotrema bahianum

752. Ocellularia brunneospora Homchant. \& Coppins, Lichenologist 34: 119 (2002) Literature: Yoshimura 1978; Homchantara and Coppins 2002; Papong et al., 2010

753. Ocellularia cerebriformis Papong, Lucking \& Lumbsch in Papong et al., Phytotaxa 189: 234 (2014)

Literature: Papong et al., 2014

754. Ocellularia chonestoma (Leight.) Zahlbr., Cat. Lich. Univ. 2: 586 (1923) [1924] Literature: Wolseley et al., 2002 
755. Ocellularia confundita Sutjaritt. \& Kalb, Herzogia 28: 545-555 (2015)

Literature: Sutjaritturakan and Kalb 2015

756. Ocellularia diacida Hale, Mycotaxon 7: 378 (1978)

Literature: Boonpragob et al., 1998; Wolseley et al., 2002

757. Ocellularia diospyrosis Homchantara \& Coppins, Lichenologist 34: 123 (2002) Literature: Yoshimura 1978; Homchantara and Coppins 2002; Wolseley et al., 2002; Papong et al., 2010

758. Ocellularia dolichotata (Nyl.) Zahlbr., Cat. Lich. Univ. 2: 589 (1923)

Literature: Vainio 1909 as Thelotrema siamense; Boonpragob et al., 1998; Wolseley et al., 2002; Kraichak et al. 2014; Lumbsch et al., 2014; Poengsungnoen et al., 2014a; Lücking et al., 2015

759. Ocellularia elixii Sutjaritt. \& Kalb, Herzogia 28: 545-555 (2015)

Literature: Sutjaritturakan and Kalb 2015

760. Ocellularia eumorpha (Stirt.) Hale, Mycotaxon 11: 136 (1980)

Literature: Wolseley et al., 2002

761. Ocellularia exigua Müll.Arg., Hedwigia 31: 284 (1892)

Literature: Lücking et al., 2015; Kraichak et al. 2014

762. Ocellularia exuta Hale, Bull. Br. Mus. nat. Hist., Bot. 8(3): 305 (1981)

Literature: Mongkolsuk et al., 2013

763. Ocellularia fumosa (Ach.) Müll.Arg., Mém. Soc. Phys. Genève 29: 7 (1887)

Literature: Kraichak et al. 2014; Papong et al., 2014; Mongkolsuk et al., 2013

764. Ocellularia flavescens Homchant. \& Coppins, Lichenologist 34: 123 (2002) Literature: Yoshimura 1978; Homchantara and Coppins 2002; Wolseley et al., 2002 Papong et al., 2010

765. Ocellularia garoana Patw. \& Nagarkar, Biovigyanam 6: 7 (1980)

Literature: Sutjaritturakan and Kalb 2015

766. Ocellularia granulifera (Kremp.) Müll.Arg., Hedwigia 31: 284 (1892)

Literature: Papong et al., 2014

767. Ocellularia guptei (Nagarkar, Sethy \& Patw.) D.D.Awasthi, Biblioth. Lichenol. 40: 3 (1991)

Literature: Boonpragob et al., 1998; Wolseley et al., 2002

768. Ocellularia interposita (Nyl.) Hale, Mycotaxon 11: 137 (1980)

Literature: Lücking et al., 2015; Kraichak et al. 2014; Lumbsch et al., 2014; Poengsungnoen et al., 2014a

769. Ocellularia inthanonensis Homchant. \& Coppins, Lichenologist 34: 123 (2002)

Literature: Yoshimura 1978; Homchantara and Coppins 2002; Wolseley et al., 2002; Papong et al., 2010

770. Ocellularia khuntanensis (Homchant. \& Coppins) Lumbsch \& Papong, in Papong et al., Lichenologist 42: 132 (2010)

Literature: Yoshimura 1978 as Myriotrema khuntanense; Homchantara and Coppins 2002; Wolseley et al., 2002; Papong et al., 2010 
771. Ocellularia klinhomii Naksuwankul, Lücking \& Lumbsch, MycoKeys 17: 55 (2016)

Literature: Naksuwankul et al., 2016

772. Ocellularia kohphangangensis Papong, Mangold \& Lucking, in Papong et al., Phytotaxa 189: 235 (2014)

Literature: Papong et al., 2014

773. Ocellularia krathingensis Homchant. \& Coppins, Lichenologist 34: 125 (2002) Literature: Yoshimura 1978; Homchantara and Coppins 2002; Wolseley et al., 2002; Papong et al., 2010; Naksuwankul et al., 2016

774. Ocellularia massalongoi (Mont.) Hale, Mycotaxon 11: 137 (1980)

Literature: Boonpragob et al., 1998; Wolseley et al., 2002

775. Ocellularia microascidium (Vain.) Zahlbr., Cat. Lich. Univ. 2: 595 (1923) [1924] Literature: Vainio 1909; Alava 1988 and Wolseley et al., 2002 all as Thelotrema microascidium

776. Ocellularia microstoma (Müll.Arg.) Hale, in Mangold et al., Flora of Australia 57: 653-659 (2009)

Literature: Yoshimura 1978 as Ocellularia peremergens; Wolseley et al., 2002 as Myriotrema microstomum; Homchantara and Coppins 2002 as Ocellularia kansriae; Papong et al., 2010

777. Ocellularia neoleucina Homchant. \& Coppins, Lichenologist 34: 126 (2002) Literature: Yoshimura 1978; Homchantara and Coppins 2002; Wolseley et al., 2002; Papong et al., 2010

778. Ocellularia neopertusariiformis Hale, Bull. Brit. Mus. (Nat. Hist.), Bot. 8: 315 (1981)

Literature: Wolseley et al., 2002

779. Ocellularia nylanderiana Hale, Phytologia 26: 414 (1973)

Literature: Boonpragob et al., 1998; Wolseley et al., 2002

780. Ocellularia palianensis Sutjaritt. \& Kalb, Phytotaxa 189: 322 (2014)

Literature: Sutjaritturakan and Kalb 2014

781. Ocellularia papillata (Leight) Zahlbr., Cat. Lich. Univ. 2: 597 (1923)

Literature: Yoshimura 1978 and Homchantara and Coppins 2002 as Myriotrema rongklaense; Boonpragob et al., 1998; Wolseley et al., 2002; Papong et al., 2010

782. Ocellularia perforata (Leight.) Müll.Arg., Hedwigia 31: 284 (1892)

Literature: Yoshimura 1978 as Myriotrema subminutum; Homchantara and Coppins 2002 and Wolseley et al., 2002 as Myriotrema khaoyaianum; Boonpragob et al., 1998; Papong et al., 2010

783. Ocellularia phatamensis Naksuwankul, Parnmen \& Lumbsch, MycoKeys 17: 56 (2016)

Literature: Naksuwankul et al., 2016

784. Ocellularia poschlodiana Sutjaritt. \& Kalb, Herzogia 28: 545-555 (2015)

Literature: Sutjaritturakan and Kalb 2015 
785. Ocellularia pertusariiformis (Leight.) Zahlbr., Cat. Lich. Univ. 2: 598 (1923) [1924]

Literature: Boonpragob et al., 1998; Wolseley et al., 2002

786. Ocellularia pluripora Hale, Bull. Brit. Mus. (Nat. Hist.), Bot. 8: 319 (1981)

Literature: Wolseley et al., 2002

787. Ocellularia pluriporoides Homchant. \& Coppins, Lichenologist 34: 127 (2002) Literature: Yoshimura 1978; Homchantara and Coppins 2002; Wolseley et al., 2002 Papong et al., 2010

788. Ocellularia pseudopapillata Papong, Mangold \& Lücking, Papong et al., Phytotaxa 198: 236 (2014)

Literature: Papong et al., 2014

789. Ocellularia punctulata (Leight) Zahlbr. Cat. Lich. Univ. 2: 599 (1923)

Literature: Boonpragob et al., 1998; Wolseley et al., 2002; Schumm and Aptroot 2012

790. Ocellularia rhicnoporoides Homchant. \& Coppins, Lichenologist 34: 128 (2002)

Literature: Yoshimura 1978; Homchantara and Coppins 2002; Wolseley et al., 2002 Papong et al., 2010; Lumbsch et al., 2014; Poengsungnoen et al., 2014a; Kraichak et al., 2014

791. Ocellularia rotundifumosa Naksukankul, Lücking \& Lumbsch, MycoKeys 17: 57 (2016)

Literature: Naksuwankul et al., 2016

792. Ocellularia salazinica Papong, Mangold \& Lücking in Papong et al., Phytotaxa198: 238 (2014)

Literature: Papong et al., 2014

793. Ocellularia sticticans Hale, Bull. Brit. Mus. (Nat. Hist.), Bot. 8: 323 (1981)

Literature: Boonpragob et al., 1998; Wolseley et al., 2002

794. Ocellularia subdolichotata Papong, Mangold \& Lumbsch in Papong et al., Phytotaxa 198: 239 (2014)

Literature: Papong et al., 2014

795. Ocellularia subgranulosa (Homchant. \& Coppins) Lumbsch \& Papong, in Papong et al., Lichenologist 42: 133 (2010)

Literature: Yoshimura 1978; Homchantara and Coppins 2002; Wolseley et al., 2002 as Myriotrema subgranulosum; Papong et al., 2010

796. Ocellularia subleucina Homchant. \& Coppins, Lichenologist 34: 129 (2002) Literature: Yoshimura 1978; Homchantara and Coppins 2002; Wolseley et al., 2002; Papong et al., 2010

797. Ocellularia terebrata (Ach.) Müll.Arg., Flora, Regensburg 70: 398 (1887) Literature: Mongkolsuk et al., 2013

798. Ocellularia thailandica Naksuwankul, Kraichak \& Lumbsch, MycoKeys 17: 58 (2016)

Literature: Naksuwankul et al., 2016 
799. Ocellularia thelotremoides (Leight.) Zahlbr. Cat. Lich. Univ. 2: 603 (1923)

Literature: Yoshimura 1978; Homchantara and Coppins 2002 as Myriotrema thailandicum; Aptroot et al., 2007; Papong et al., 2010; Kraichak et al. 2014

800. Ocellularia urceolaris Ach., Syst. Orb. Veg. (Lundae) 4: 242 (1827)

Literature: Boonpragob et al., 1998; Wolseley et al., 2002 as Ocellularia leucina

801. Ocellularia violacea Räsänen, Suom. Elain-ja Kasvit. Seuran Van. Tiedon. Pöytäkirjat 3: 184 (1949)

Literature: Papong et al., 2014

802. Ocellularia viridipallens Müll.Arg., Flora, Regensburg 70: 397 (1887)

Literature: Kraichak et al., 2014; Papong et al., 2014

803. Ocellularia wolseleyana Homchant. \& Coppings, Lichenologist 34: 133-140 (2010)

Literature: Yoshimura 1978; Homchantara and Coppins 2002; Wolseley et al., 2002; Papong et al., 2010

804. Ocellularia xanthostromiza (Nyl.) Zahlbr., Cat. Lich. Univers. 2: 604 (1923) Literature: Kraichak et al., 2014

805. Ocellularia zamboangensis (Vain.) Zahlbr., Cat. Lich. Univers. 2: 604 (1923) [1924]

Literature: Kraichak et al., 2014

806. Ochrolechia africana Vain., Ann. Univ. Fenn. Åbo., A 2: 3 (1926)

Literature: Aptroot et al., 2007

807. Opegrapha apomelaenna A. Massal., Verh. K. K. Zool.-Bot. Ges. Wien 21:864 (1871)

Literature: Ertz 2009

808. Opegrapha astraea Tuck. Lich. Calif.: 33 (1866)

Literature: Ertz 2009

809. Opegrapha dekeselii Ertz, Biblioth. Lichenol. 102: 49 (2009)

Literature: Ertz 2009; Schumm and Aptroot 2012

810. Opegrapha gyrocarpa Flot., Flora 8: 345 (1825)

Literature: Aptroot et al., 2007

811. Opegrapha herbarum Mont. Arch. Bot. Guillemmin 2: 302 (1833)

Literature: Ertz 2009

812. Opegrapha lambinonii Sérus., Lejeunia 90: 2 (1978)

Literature: Wolseley et al., 2002

813. Opegrapha prosodea Ach., Methodus (Acharius): 22 (1803)

Literature: Paulson 1930; Wolseley et al., 2002

814. Opegrapha robusta Vain., Bot. Tidsskr. 29: 137 (1909)

Literature: Vainio 1909; Alava 1988; Wolseley et al., 2002

815. Opegrapha subvulgata Nyl., Flora 52: 71 (1869)

Literature: Vainio 1909; Wolseley et al., 2002

816. Opegrapha vegae R.Sant., Symb. Bot. Upsal. 12: 99 (1952)

Literature: Papong et al., 2007 
817. Opegrapha velata Vain., Cat. Afr. Pl. 2: 444 (1901)

Literature: Papong et al., 2007

818. Opegrapha viridis (Ach.) Behlen \& Desberger, Naturgesch. und Beschreib. Deutsch. Forst.-Kryptog.: 110 (1835)

Literature: Ertz 2009

819. Opegrapha viridistellata Sérus., Lücking \& Sparrius, Mycotaxon 104: 223 (2008)

Literature: Schumm and Aptroot 2012

820. Pallidogramme chlorocarpoides (Nyl.) Staiger, Kalb \& Lücking, in Lücking et al., Fieldiana, Bot. 38: 9 (2008)

Literature: Vainio 1909; 1921 as Graphis chlorocarpoides; Wolseley et al., 2002 as Phaeographina chlorocarpoides; Poengsungnoen et al., 2010 as Hemithecium chlorocarpoides

821. Pallidogramme chrysenteron (Mont.) Staiger, Kalb \& Lücking, in Lücking, Chaves, Sipman, Umaña \& Aptroot, Fieldiana, Bot. 38(no. 1549): 9 (2008) Literature: Wolseley et al., 2002 as Phaeographina chrysenteron; Paulson 1930 as Phaeographina chrysentera; Poengsungnoen et al., 2010 as Hemithecium chrysenteron; Mongkolsuk et al., 2013

822. Pannaria dispartita (Nyl.) Vain., Suom. Elain-ja Kasvit. Seuran Van. Julk. 1: 45 (1921).

Literature: Vainio 1921; Wolseley et al., 2002

823. Parainoa subconcolor (Anzi) Resl \& T. Sprib., in Resl et al., Fung. Div. 73: 254 (2015)

Literature: Aptroot et al., 2007 as Trapelia subconcolor

824. Parmelinella chozoubae (Kr.P.Singh \& G.P.Sinha) Elix \& Pooprang, Mycotaxon 71: 115 (1998)

Literature: Pooprang et al., 1999; Wolseley et al., 2002; Buaruang et al., 2009; Louwhoff et al., 2012

825. Parmelinella salacinifera (Hale) Marcelli \& Benatti, in Benatti, Mycosphere 5: 780 (2014)

Literature: Hale 1976c as Pseudoparmelia salacinifera; Wolseley and AguirreHudson 1995; Moon et al., 2000a; Wolseley et al., 2002; Louwhoff et al., 2012 as Canoparmelia salacinifera

826. Parmelinella simplicior (Hale) Elix \& Hale, Mycotaxon29: 242 (1987)

Literature: Moon et al., 2000a; Wolseley et al., 2002; Louwhoff et al., 2012

827. Parmelinella wallichiana (Taylor) Elix \& Hale, Mycotaxon 29: 242 (1987) Literature: Hale 1976b as Parmelina wallichiana; Moon et al., 2000a; Wolseley et al., 2002; Buaruang et al., 2009; Wolseley and Aguirre-Hudson 1995, 1997a, 1997b; Louwhoff et al., 2012

828. Parmeliopsis ambigua (Wulfen) Nyl., Syn. Meth. Lich. 2: 54 (1863) Literature: Pooprang et al., 1999; Wolseley et al., 2002; Louwhoff et al., 2012

829. Parmotrema abessinicum (Nyl. ex Kremp.) Hale, Phytologia 28: 334 (1974) Literature: Pooprang et al., 1999; Wolseley et al., 2002; Louwhoff et al., 2012 
830. Parmotrema adspersum (Vain.) Elix, Mycotaxon 81: 255 (2002)

Literature: Vainio 1909; Alava 1988 as Parmelia adspersa; Hale 1976c as Pseudoparmelia adspersa; Wolseley et al., 2002 as Canoparmelia adspersa

831. Parmotrema amaniense (J.Steiner \& Zahlbr.) Krog \& Swinscow, Lichenologist 15: 129 (1983)

Literature: Wolseley and Aguirre-Hudson 1997a; Wolseley et al., 2002

832. Parmotrema andinum (Müll.Arg.) Hale, Phytologia 28: 334 (1974)

Literature: Hale 1965a as Parmelia andina; Wolseley et al., 2002; Louwhoff et al., 2012

833. Parmotrema austrosinense (Zahlbr.) Hale, Phytologia 28: 335 (1974)

Literature: Moon et al., 2000a; Wolseley et al., 2002

834. Parmotrema breviciliatum (Hale) Hale, Phytologia 28: 335 (1974) Literature: Hale 1965a as Parmelia breviciliata

835. Parmotrema cetratum (Ach.) Hale, Phytologia 28: 335 (1974)

Literature: Louwhoff et al., 2012

836. Parmotrema cooperi (J.Steiner \& Zahlbr.) Sérus., Bryologist 87: 4 (1984)

Literature: Saipunkaew et al., 2007; Louwhoff et al., 2012

837. Parmotrema corniculans (Nyl.) Hale, Phytologia 28: 335 (1974)

Literature: Pooprang et al., 1999; Wolseley et al., 2002; Louwhoff et al., 2012

838. Parmotrema crinitum (Ach.) M.Choisy, Bull. Mens. Soc. Linn. Soc. Bot. Lyon 21: 175 (1952)

Literature: Mongkolsuk et al., 1996; Wolseley et al., 2002

839. Parmotrema cristiferum (Taylor) Hale, Phytologia 28: 335 (1974)

Literature: Hale 1965b as Parmelia andina; Vainio 1909 as Parmelia latissima var. cristifera; Boonpragob et al., 1998; Moon et al., 2000a; Wolseley et al., 2002; Buaruang et al., 2009b; Louwhoff et al., 2012

840. Parmotrema dilatatum (Vain.) Hale, Phytologia 28: 335 (1974)

Literature: Boonpragob et al., 1998; Moon et al., 2000a; Wolseley et al., 2002; Louwhoff et al., 2012

841. Parmotrema dominicanum (Vain.) Hale, Phytologia 28: 336 (1974)

Literature: Moon et al., 2000a; Wolseley et al., 2002

842. Parmotrema elacinulatum (Kurok.) Streimann, Biblioth. Lichenol. 22: 94 (1986)

Literature: Pooprang et al., 1999; Wolseley et al., 2002

843. Parmotrema eunetum (Stirt.) Hale, Phytologia 28: 336 (1974)

Literature: Moon et al., 2000a; Wolseley et al., 2002; Louwhoff et al., 2012

844. Parmotrema euplectinum Elix, Noich. \& Wolsaley, in Noicharoen et al., Mycotaxon 85: 326 (2003)

Literature: Noicharoen et al., 2003

845. Parmotrema eurysacum (Hue) Hale, Phytologia 28: 336 (1974)

Literature: Wolseley et al., 2002

846. Parmotrema explanatum (Hale) Hale, Phytologia 28:337 (1974)

Literature: Hale 1965b as Parmelia explanata; Moon et al., 2000a; Wolseley et al., 2002 
847. Parmotrema gardneri (C.W.Dodge) Sérus., Bryologist 87:5 (1984)

Literature: Wolseley and Aguirre-Hudson 1997a; Wolseley et al., 2002; Saipunkaew et al., 2007; Buaruang et al., 2009; Louwhoff et al., 2012

848. Parmotrema hababianum (Gyeln.) Hale, Phytologia 28: 336 (1974)

Literature: Pooprang et al., 1999; Wolseley et al., 2002; Louwhoff et al., 2012

849. Parmotrema hypotropum (Nyl.) Hale, Phytologia 28: 337 (1974)

Literature: Wolseley et al., 2002

850. Parmotrema incrassatum Hale ex DePriest \& B.W.Hale, Mycotaxon 67: 207 (1998)

Literature: Pooprang et al., 1999; Wolseley et al., 2002; Saipunkaew et al., 2007; Louwhoff et al., 2012

851. Parmotrema latissimum (Fée) Hale, Phytologia 28: 337 (1974)

Literature: Louwhoff et al., 2012

852. Parmotrema lobulascens (J.Steiner) Hale, Phytologia 28: 337 (1974)

Literature: Boonpragob et al., 1998; Wolseley et al., 2002; Louwhoff et al., 2012

853. Parmotrema lophogenum (Abbayes) Hale, Phytologia 28: 337 (1974)

Literature: Mongkolsuk et al., 2013

854. Parmotrema maclayanum (Müll.Arg.) Hale, Phytologia 28: 337 (1974)

Literature: Buaruang et al., 2009; Moon et al., 2000a; Wolseley et al., 2002; Louwhoff et al., 2012

855. Parmotrema mellissii (C.W.Dodge) Hale, Phytologia 28: 337 (1974)

Literature: Moon et al., 2000a; Wolseley et al., 2002; Louwhoff et al., 2012

856. Parmotrema merrillii (Vain.) Hale, Phytologia 28: 337 (1974)

Literature: Louwhoff et al., 2012

857. Parmotrema nanfongense (Kurok.) DePriest \& B.W.Hale, Mycotaxon 67: 204 (1998)

Literature: Moon et al., 2000a; Wolseley et al., 2002; Louwhoff et al., 2012

858. Parmotrema nilgherrense (Nyl.) Hale, Phytologia 28: 338 (1974)

Literature: Hale 1965a as Parmelia nilgherrense; Vainio 1921; Alava 1988 as

Parmelia siamensis; Boonpragob et al., 1998; Wolseley and Aguirre-Hudson 1997a; Wolseley et al., 2002

859. Parmotrema overeemii (Zahlbr.) Elix, Australas. Lichenol. 43: 23 (1998)

Literature: Pooprang et al., 1999; Wolseley et al., 2002; Louwhoff et al., 2012

860. Parmotrema pancheri (Hue) Hale, Phytologia 28: 338 (1974)

Literature: Moon et al., 2000a; Wolseley et al., 2002; Louwhoff et al., 2012

861. Parmotrema perforatum (Jacq.) A.Massal., Atti Inst. Veneto Sci. lett., ed Arti, Sér. 3 5: 248 (1860)

Literature: Mongkolsuk et al., 2013

862. Parmotrema perlatum (Huds.) M.Choisy, Bull. Mens. Soc. Linn. Soc. Bot. Lyon 21: 174 (1952)

Literature: Vainio 1909 as Parmelia perlata; Boonpragob et al., 1998 ; Wolseley et al., 2002 as Parmotrema chinense; Louwhoff et al., 2012 
863. Parmotrema permutatum (Stirt.) Hale, Phytologia 28:338 (1974)

Literature: Hale 1965a as Parmelia permutata; Moon et al., 2000a; Wolseley et al., 2002; Buaruang et al., 2009b; Louwhoff et al., 2012

864. Parmotrema planatilobatum (Hale) Hale, Phytologia 28:338 (1974)

Literature: Pooprang et al., 1999; Wolseley et al., 2002

865. Parmotrema platyphyllinum (Vain.) Elix, Australas. Lichenol. 42: 22-27 (1998) Literature: Vainio 1909; Alava 1988 as Parmelia platyphyllina; Moon et al., 2000a; Wolseley et al., 2002; Saipunkaew et al., 2007; Louwhoff et al., 2012

866. Parmotrema poolii (C.W.Dodge) Krog \& Swinscow, Lichenologist 15: 130 (1983) Literature: Moon et al., 2000a; Wolseley et al., 2002; Buaruang et al., 2009; Louwhoff et al., 2012

867. Parmotrema praesorediosum (Nyl.) Hale, Phytologia 28:338 (1974)

Literature: Moon et al., 2000a; Wolseley et al., 2002; Saipunkaew et al., 2007; Buaruang et al., 2009; Louwhoff et al., 2012; Schumm and Aptroot 2012

868. Parmotrema procerum (J.Steiner \& Zahlbr.) Hale, Phytologia 28: 338 (1974) Literature: Hale 1965a as Parmelia procera; Boonpragob et al., 1998; Wolseley et al., 2002; Louwhoff et al., 2012

869. Parmotrema pseudocrinitum (Abbayes) Hale, Phytologia 28:338 (1974) Literature: Moon et al., 2000a; Buaruang et al., 2009

870. Parmotrema pseudonilgherrense (Asahina) Hale, Mycotaxon 5: 441 (1977) Literature: Pooprang et al., 1999; Wolseley et al., 2002

871. Parmotrema rampoddense (Nyl.) Hale, Phytologia 28:338 (1974)

Literature: Mongkolsuk et al., 1996; Moon et al., 2000a; Wolseley et al., 2002; Saipunkaew et al., 2007; Buaruang et al., 2009; Louwhoff et al., 2012

872. Parmotrema reticulatum (Taylor) M.Choisy, Bull. Mens. Soc. Linn. Soc. Bot. Lyon 21: 148 (1952)

Literature: Satô 1962; Vainio 1921 as Parmelia cetrata var. sorediifera; Wolseley and Aguirre-Hudson 1995; 1997a; Moon et al., 2000a; Wolseley et al., 2002 as Rimelia clavulifera and Rimelia reticulata; Buaruang et al., 2009a; Buaruang et al., 2009b; Louwhoff et al., 2012; Mongkolsuk et al., 2013

873. Parmotrema robustum (Degel.) Hale, Phytologia 28: 338 (1974)

Literature: Moon et al., 2000a; Wolseley et al., 2002

874. Parmotrema rubromarginatum Elix \& Pooprang, Mycotaxon 72:115 (1999)

Literature: Pooprang et al., 1999; Wolseley et al., 2002

875. Parmotrema saccatilobum (Taylor) Hale, Phytologia 28:339 (1974)

Literature: Moon et al., 2000a; Wolseley et al., 2002; Saipunkaew et al., 2007; Schumm and Aptroot 2012; Louwhoff et al., 2012

876. Parmotrema sancti-angelii (Lynge) Hale, Phytologia 28:339 (1974)

Literature: Moon et al., 2000a; Wolseley et al., 2002; Buaruang et al., 2009; Louwhoff et al., 2012

877. Parmotrema stuppeum (Taylor) Hale, Phytologia 28: 339 (1974)

Literature: Vainio 1909 as Parmelia perforata v. claudelii; Moon et al., 2000a; Wolseley et al., 2002 
878. Parmotrema subarnoldii (Abbayes) Hale, Phytologia 28:339 (1974)

Literature: Pooprang et al., 1999; Wolseley et al., 2002; Louwhoff et al., 2012

879. Parmotrema subcaperatum (Kremp.) Hale, Phytologia 28: 339 (1974)

Literature: Louwhoff et al., 2012

880. Parmotrema submarginale (Michx.) DePriest \& B.W.Hale, Mycotaxon 67: 208 (1998)

Literature: Mongkolsuk et al., 1996 as Parmelia michauxianum; Wolseley et al., 2002

881. Parmotrema subsumptum (Nyl.) Hale. Mycotaxon 5: 434 (1977)

Literature: Wolseley and Aguirre-Hudson 1995; Moon et al., 2000a; Wolseley et al., 2002 as Canomaculina subsumpta; Aptroot et al., 2007; Louwhoff et al., 2012

882. Parmotrema subtinctorium (Zahlbr.) Hale, Phytologia 28: 39 (1974)

Literature: Mongkolsuk et al., 1996, Pooprang et al., 1999; Wolseley et al., 2002 as Canomaculina subtinctoria

883. Parmotrema sulphuratum (Nees \& Flot.) Hale, Phytologia 28:339 (1974)

Literature: Pooprang et al., 1999; Wolseley et al., 2002; Louwhoff et al., 2012

884. Parmotrema thailandicum Elix \& Pooprang, in Pooprang et al., Mycotaxon 71: 117 (1999)

Literature: Pooprang et al., 1999; Wolseley et al., 2002; Louwhoff et al., 2012

885. Parmotrema thomsonii (Stirt.) A.Crespo, Divakar \& Elix, in Crespo et al., Taxon 59: 1746 (2010)

Literature: Wolseley and Aguirre-Hudson 1995; Wolseley et al., 2002 as Parmelaria thomsonii

886. Parmotrema tinctorum (Despr. ex Nyl.) Hale, Phytologia 28:339 (1974)

Literature: Paulson 1930; Satô 1962; Vainio 1921 as Parmelia tinctorum; Wolseley and Aguirre-Hudson 1997a; Moon et al., 2000a; Wolseley et al., 2002; Saipunkaew et al., 2007; Buaruang et al., 2009; Louwhoff et al., 2012; Schumm and Aptroot 2012; Mongkolsuk et al., 2013

887. Parmotrema usambarense (J.Steiner \& Zahlbr.) Krog \& Swinscow, Lichenologist 19: 424 (1987)

Literature: Hale 1976b; Moon et al., 2000a; Wolseley et al., 2002 as Parmelina usambarensis

888. Parmotrema zollingeri (Hepp) Hale, Phytologia 28:339 (1974)

Literature: Hale 1965a as Parmelia zolingeri; Moon et al., 2000a; Wolseley et al., 2002

889. Peltigera pindarensis D.D.Awasthi \& M.Joshi, Kavaka 10: 58 (1982)

Literature: Aptroot et al., 2007

890. Peltula corticola Büdel \& R. Sant., in Büdel, Biblioth. Lichenol. 23: 79 (1987) Literature: Schumm and Aptroot 2012 as Phyllopeltula corticola

891. Peltula obscurans (Nyl.) Gyeln., Feddes Repert. Spec. Nov. Regni Veg. 38: 308 (1935)

Literature: Aptroot et al., 2007 
892. Pertusaria alboaspera var. deficiens Jariangpr. \& A.W.Archer, in Jariangprasert et al., Mycotaxon 85: 290 (2003)

Literature: Jariangprasert et al., 2003

893. Pertusaria alboaspera var. disflavens Jariangpr., Mycotaxon 96: 109 (2006)

Literature: Jariangprasert 2006

894. Pertusaria alboaspera var. tetraspora Jariangpr., Mycotaxon 91: 280 (2005)

Literature: Jariangprasert and Anusarnsunthorn 2005

895. Pertusaria allomicrostoma Jariangpr., Telopea 12: 264 (2008)

Literature: Elix et al., 2008

896. Pertusaria allothwaitesii Jariangpr. \& A.W.Archer, in Jariangprasert et al., Mycotaxon 89: 124 (2004)

Literature: Jariangprasert et al., 2004

897. Pertusaria archeri Jariangpr., Mycotaxon 91: 281 (2005)

Literature: Jariangprasert and Anusarnsunthorn 2005

898. Pertusaria asiana Vain., Ann. Bot. Soc. Zool.-Bot. Fenn. "Vanamo" 1: 44 (1921)

Literature: Vainio 1921; Alava 1988; Wolseley et al., 2002

899. Pertusaria bengalensis Vain., Hedwigia 56: 169 (1907)

Literature: Vainio 1907, 1909; Alava 1988; Wolseley et al., 2002

900. Pertusaria bokluensis Jariangpr., Mycotaxon 91: 281 (2005)

Literature: Jariangprasert and Anusarnsunthorn 2005

901. Pertusaria cicatricosa Müll.Arg., Proc. Roy. Soc. Edinburgh 11: 461 (1882)

Literature: Aptroot et al., 2007

902. Pertusaria confluentica Jariangpr. \& Elix, Telopea 12: 265 (2008)

Literature: Elix et al., 2008

903. Pertusaria elixii Jariangpr., Mycotaxon 91: 282 (2005)

Literature: Jariangprasert and Anusarnsunthorn 2005

904. Pertusaria hylocola Jariangpr. \& A.W.Archer, in Jariangprasert et al., Mycotaxon 85: 292 (2003)

Literature: Jariangprasert et al., 2003

905. Pertusaria hypostictica Jariangpr., Mycotaxon 91: 283 (2005)

Literature: Jariangprasert and Anusarnsunthorn 2005

906. Pertusaria inthanonensis Jariangpr., Mycotaxon 91: 283 (2005)

Literature: Jariangprasert and Anusarnsunthorn 2005

907. Pertusaria isidiosa A.W.Archer, Mycotaxon 41: 228 (1991)

Literature: Wolseley and Aguirre-Hudson 1997a

908. Pertusaria kansriae Jariangpr., Mycotaxon 91: 284 (2005)

Literature: Jariangprasert and Anusarnsunthorn 2005

909. Pertusaria kansriae var. stictica Jariangpr., Mycotaxon 96: 110 (2006)

Literature: Jariangprasert 2006

910. Pertusaria krabiensis Jariangpr., Mycotaxon 91: 286 (2005)

Literature: Jariangprasert and Anusarnsunthorn 2005 
911. Pertusaria lansangensis Jariangpr. \& A.W.Archer, in Jariangprasert et al., Mycotaxon 89: 125 (2004)

Literature: Jariangprasert et al., 2004

912. Pertusaria litchicola Jariangpr. \& A.W.Archer, in Jariangprasert et al., Mycotaxon 85: 292 (2003)

Literature: Jariangprasert et al., 2003

913. Pertusaria loeiensi Jariangpr., Mycotaxon 91: 286 (2005)

Literature: Jariangprasert and Anusarnsunthorn 2005

914. Pertusaria methylstenosporica Jariangpr., Mycotaxon 96: 111 (2006)

Literature: Jariangprasert 2006

915. Pertusaria microstoma var. isidiata Jariangpr., Mycotaxon 96: 111 (2006) Literature: Jariangprasert 2006

916. Pertusaria montpittensis A.W.Archer, in Elix et al., Proc. Linn. Soc. New South Wales 113: 65 (1992)

Literature: Schumm and Aptroot 2012

917. Pertusaria nahaeoensis Jariangpr. \& A.W.Archer, in Jariangprasert et al., Mycotaxon 83: 355 (2002)

Literature: Jariangprasert et al., 2002

918. Pertusaria nanensis Jariangpr. \& A.W.Archer, in Jariangprasert et al., Mycotaxon 89: 126 (2004)

Literature: Jariangprasert et al., 2004

919. Pertusaria neoknightiana Jariangpr., Mycotaxon 96: 112 (2006)

Literature: Jariangprasert 2006

920. Pertusaria omkoiensis Jariangpr. \& A.W.Archer, in Jariangprasert et al., Mycotaxon 83: 355 (2002)

Literature: Jariangprasert et al., 2002

921. Pertusaria orientalis Jariangpr., Mycotaxon 96: 113 (2006)

Literature: Jariangprasert 2006

922. Pertusaria parameeana Jariangpr., Mycotaxon 96: 115 (2006)

Literature: Jariangprasert 2006

923. Pertusaria pertusella Müll.Arg., Flora 67: 283 (1884)

Literature: Aptroot et al., 2007

924. Pertusaria phulhuangensis Jariangpr., Mycotaxon 96: 116 (2006)

Literature: Jariangprasert 2006

925. Pertusaria phusoidaoensis Jariangpr., Telopea 12: 269 (2008)

Literature: Elix et al., 2008

926. Pertusaria pilosula var. abditiva Jariangpr., Mycotaxon 96: 117 (2006)

Literature: Jariangprasert 2006

927. Pertusaria platycarpa Jariangpr., Mycotaxon 96: 118 (2006)

Literature: Jariangprasert 2006

928. Pertusaria pustulata (Ach.) Duby, Bot. Gall. 2: 673 (1830)

Literature: Vainio 1909; Wolseley et al., 2002 
929. Pertusaria siamensis Jariangpr., Mycotaxon 91: 287 (2005)

Literature: Jariangprasert and Anusarnsunthorn 2005

930. Pertusaria sphaerulifera Vain., Hedwigia 46: 169 (1907)

Literature: Vainio 1909; Alava 1988

931. Pertusaria subcopelandii Jariangpr., Mycotaxon 96: 118 (2006)

Literature: Jariangprasert 2006

932. Pertusaria subnegans Vain., Hedwigia 46: 170 (1907)

Literature: Vainio 1909; Alava 1988; Wolseley et al., 2002

933. Pertusaria subplanaica var. stictica Jariangpr., Mycotaxon 96: 120 (2006)

Literature: Jariangprasert 2006

934. Pertusaria subplanaica var. tetraspora Jariangpr. \& A.W.Archer, in Jariangprasert et al., Mycotaxon 85: 293 (2003)

Literature: Jariangprasert et al., 2003

935. Pertusaria takensis Jariangpr. \& A.W.Archer, in Jariangprasert et al., Mycotaxon 89: 128 (2004)

Literature: Jariangprasert et al., 2004

936. Pertusaria thailandica Jariangpr., Mycotaxon 91: 288 (2005)

Literature: Jariangprasert and Anusarnsunthorn 2005

937. Pertusaria uttaraditensis Jariangpr., Mycotaxon 91: 291 (2005)

Literature: Jariangprasert and Anusarnsunthorn 2005

938. Phaeographis brasiliensis (A.Massal.) Kalb \& Matthes-Leicht, Biblioth. Lichenol. 78: 148 (2001)

Literature: Poengsungnoen et al., 2010

939. Phaeographis caesiodiscoides Mongkolsuk \& Kalb, Phytotaxa 189: 255-267 (2014)

Literature: Poengsungnoen et al., 2014b

940. Phaeographis caesioradians (Leight.) Kalb, Lich. Neotrop. 13: 13 (2001)

Literature: Mongkolsuk and Poengsungnoen 2012

941. Phaeographis dendroides (Leight.) Müll.Arg., Flora 65: 208. (1882)

Literature: Poengsungnoen et al., 2010

942. Phaeographis exaltata (Mont. \& Bosch.) Müll.Arg., Flora 65: 336. (1882)

Literature: Vainio 1909 as Graphis diversa; Wolseley et al., 2002

943. Phaeographis hypoglauca (Kremp.) Zahlbr., Cat. Lich. Univ. 2: 374 (1923).

Literature: Vainio 1909 as Graphis persimilis; Pitakpong et al., 2015

944. Phaeographis hypoglaucoides Kr.P.Singh \& D.D.Awasthi, Bull. Bot. Surv. India 21: 109 (1981)

Literature: Lumbsch et al., 2014

945. Phaeographis intricans Kr.P.Singh \& D.D.Awasthi, Bull. Bot. Surv. India 21: 109 (1981)

Literature: Aptroot et al., 2007; Poengsungnoen et al., 2010; Lumbsch et al., 2014

946. Phaeographis kalbii Staiger, Biblioth. Lichenol. 85: 332 (2002)

Literature: Aptroot et al., 2007 
947. Phaeographis loeiensis Boonpragob, Manoch \& Poengs., Phytotaxa 189: 255267 (2014)

Literature: Poengsungnoen et al., 2014b

948. Phaeographis leiogrammodes (Kremp.) Müll.Arg., Nuovo Giorn. Bot. Ital. 23: 397 (1891)

Literature: Aptroot et al., 2007

949. Phaeographis nardiensis A.W.Archer, Telopea 9: 674 (2001)

Literature: Poengsungnoen et al., 2010

950. Phaeographis neotricosoides Poengs. \& Kalb, Phytotaxa 189: 255-267 (2014)

Literature: Poengsungnoen et al., 2014b

951. Phaeographis nylanderi (Vain.) Zahlbr., Cat. Lich. Univ. 2: 382 (1923) [1924] Literature: Vainio 1909 as Graphina nylanderi; Wolseley et al., 2002

952. Phaeographis phurueaensis Poengs. \& Kalb, Phytotaxa 189: 255-267 (2014) Literature: Poengsungnoen et al., 2014b

953. Phaeographis rugulosa (Vain.) Zahlbr., Cat. Lich Univ. 2: 444 (1923) Literature: Alava 1909; Vainio 1921 as Graphis rugulosa; Wolseley et al., 2002

954. Phaeographis schizolomoides Poengs. \& Kalb, Phytotaxa 189: 255-267 (2014) Literature: Poengsungnoen et al., 2014b

955. Phaeographis scalpturata (Ach.) Staiger, Biblioth. Lichenol. 85: 345 (2002) Literature: Vainio 1921 as Graphis sculpturata; Wolseley et al., 2002 as Phaeographina scalpturata

956. Phaeographis siamensis Poengs. \& Kalb, Phytotaxa 189: 255-267 (2014)

Literature: Poengsungnoen et al., 2014b

957. Phaeographis subintricata Müll. Arg., Bull. Herb. Boissier 3: 320 (1895)

Literature: Vainio 1909; Wolseley et al., 2002 as Phaeographina subintricata

958. Phaeographis subtigrina (Vain.) Zahlbr. Cat. Lich. Univ. 2: 387 (1923)

Literature: Vainio 1909; Alava 1988 as Graphina subtigrina; Wolseley et al., 2002

959. Phaeographopsis indica (Patw. \& Nagarkar) Sipman \& Aptroot, in Aptroot et al., Fungal Diversity 24: 114 (2007)

Literature: Aptroot et al., 2007

960. Phaeophyscia hispidula (Ach.) Essl., Mycotaxon 7: 305 (1978)

Literature: Paulson 1930 as Physcia setosa; Wolseley et al., 2002

961. Phaeophyscia limbata (Poelt) Kashiw., Bull. Natl. Sci. Mus., Tokyo, B. 10: 129 (1984)

Literature: Aptroot et al., 2007

962. Phaeophyscia orbicularis (Neck.) Moberg, Symb. Bot. Upsal. 22: 44 (1977)

Literature: Mongkolsuk and Meesim 2012

963. Phaeophyscia primaria (Poelt) Trass, Folia Cryptog. Estonica. 15: 2 (1981)

Literature: Schumm and Aptroot 2012

964. Phlyctis himalayensis (Nyl.) D.D.Awasthi, Lichenology in Indian Subcontinent: 15 (2000)

Literature: Vainio 1921 as Phylctis polyphragmia; Wolseley et al., 2002 as Phlyctella himalayensis 
965. Phlyctis neozelandica Nyl., Flora 52: 121 (1869)

Literature: Paulson 1930 as Phlyctella neozelandica; Wolseley et al., 2002

966. Phlyctis uncinata Stirt., J. Linn. Soc., Bot. 14: 464 (1875)

Literature: Aptroot et al., 2007 as Phlyctella uncinata

967. Phyllogyalidea epiphylla (Vězda) Lücking \& Aptroot, Fl. Neotrop., Monogr. 103: 383 (2008)

Literature: Aptroot et al., 2007

968. Phyllopsora buettneri (Müll.Arg.) Zahlbr., Cat. Lich. Univ. 4: 396 (1926) [1927] Literature: Wolseley et al., 1994

969. Phyllopsora furfuracea Zahlbr., in Engler \& Prantl, Nat. Pflanzenfam. Nachtr. [Engler \& Prantl] 1: 138 (1905)

Literature: Wolseley and Aguirre-Hudson 1997b; Boonpragob et al., 1998;

Wolseley et al., 2002

970. Phyllopsora viridis Paulson, J. Siam Soc., Nat. Hist., 8: 101 (1930)

Literature: Paulson 1930

971. Physcia atrostriata Moberg, Nordic J. Bot. 6: 853 (1986)

Literature: Aptroot et al., 2007; Meesim and Mongkolsuk 2010a; Van den Boom et al., 2014

972. Physcia crispa Nyl., Syn. Meth. Lich 1: 432 (1860)

Literature: Saipunkaew et al., 2007

973. Physcia erumpens Moberg, Nordic J. Bot. 6: 856 (1986)

Literature: Meesim and Mongkolsuk 2010a; Mongkolsuk and Meesim 2012

974. Physcia integrata Nyl., Syn. Meth. Lich. 1: 424 (1860)

Literature: Aptroot et al., 2007; Meesim and Mongkolsuk 2010a; Mongkolsuk and Meesim 2012; Van den Boom et al., 2014

975. Physcia krogiae Moberg, Nordic J. Bot. 6: 858 (1986)

Literature: Saipunkaew et al., 2007

976. Physcia poncinsii Hue, Bull. Soc. Bot. France: 10 (1917)

Literature: Saipunkaew et al., 2007

977. Physcia sorediosa (Vain.) Lynge, Skr. Vidensk.-Selsk. Christiania, Math.Naturvidensk. KL. no. 16: 27 (1924)

Literature: Paulson 1930; Wolseley et al., 2002; Van den Boom et al., 2014

978. Physcia undulata Moberg, Nordic J. Bot. 6: 861 (1986)

Literature: Vainio 1909 as Physcia crispa v. mollescens; Mongkolsuk et al., 2015 and Wolseley et al., 2002 as Heterodermia albicans; Meesim and Mongkolsuk 2010a; Mongkolsuk and Meesim 2012

979. Physcidia cylindrophora (Taylor) Hue, Bull. Soc. Linn. Normandie 1: 97 (1908) Literature: Boonpragob et al., 1998; Wolseley et al., 2002; Aptroot et al., 2007

980. Physcidia squamulosa Tuck., Proc. Amer. Acad. Arts 5: 401 (1862) [1860] Literature: Aptroot et al., 2007

981. Physcidia wrightii (Tuck.) Tuck., Proc. Amer. Acad. Arts Sci. 5: 400 (1862) Literature: Wolseley et al., 1994; Wolseley and Aguirre-Hudson 1995; 1997a; 1997b; Boonpragob et al., 1998; Wolseley et al., 2002; Aptroot et al., 2007 
982. Physciella chloantha (Ach.) Essl., Mycologia 78(1): 94 (1986)

Literature: Meesim and Mongkolsuk 2010a; Mongkolsuk and Meesim 2012 as Phaeophyscia chloantha

983. Physma byrsaeum (Ach.) Tuck., Syn. N. Amer. Lich. 1:115 (1882)

Literature: Wolseley and Aguirre-Hudson 1995; Wolseley et al., 2002; Rangsiruji et al., 2016

984. Physma plicatum Hue, Bull. Soc. Linn. Normandie 9: 136 (1906)

Literature: Vainio 1909; Wolseley et al., 2002

985. Physma radians Vain., Ann. Acad. Sci. fenn., Ser. A 6: 45 (1921)

Literature: Ohmura et al., 2016

986. Piccolia conspersa (Fée) Hafellner, Biblioth. Lichenol. 58: 109 (1995)

Literature: Aptroot et al., 2007

987. Placynthium nigrum (Huds.) Gray, Nat. Arr. Brit. Pl. 2: 395 (1821)

Literature: Aptroot et al., 2007

988. Platismatia erosa W.L.Culb. \& C.F.Culb., Contr. U.S. Natl. Herb. 34: 526 (1968) Literature: Wolseley and Aguirre-Hudson 1997a; Wolseley et al., 2002

989. Platygramme caesiopruinosa (Fée) Fée, Bull. Soc. bot. Fr. 21: 30 (1874)

Literature: Vainio 1921; Wolseley et al., 2002 as Phaeographina caesiopruinosa; Poengsungnoen et al., 2010 as Graphis caesiopruinosa

990. Platygramme microspora Sutjaritt. \& Kalb, Phytotaxa 189: 322 (2014)

Literature: Sutjaritturakan and Kalb 2014

991. Platygramme platyloma (Müll. Arg.) M. Nakan. \& Kashiw., in Nakanishi, Kashiwadani \& Moon, Bull. Natn. Sci. Mus., Tokyo, B 29(2): 89 (2003)

Literature: Lumbsch et al., 2014 as Platygramme impudica

992. Platygramme pudica (Mont. \& Bosch) M.Nakan. \& Kashiw., in Nakanishi et al., Bull. Natl. Sci. Mus., Tokyo, B. 29: 89 (2003)

Literature: Poengsungnoen et al., 2010

993. Platygramme subarechavaletae Poengs. \& Kalb, Phytotaxa 189: 255-267 (2014) Literature: Poengsungnoen et al., 2014b

994. Platythecium allosporellum (Nyl.) Staiger, Biblioth. Lichenol. 85: 377 (2002) Literature: Aptroot et al., 2007

995. Platythecium colliculosum (Mont.) Staiger, Biblioth. Lichenol. 85: 380 (2002) Literature: Aptroot et al., 2007

996. Platythecium dimorphodes (Nyl.) Staiger, Biblioth. Lichenol. 85: 383 (2002) Literature: Aptroot et al., 2007; Nakanishi et al., 2001 as Graphina dimorphodes

997. Platythecium grammitis (Fée) Staiger, Biblioth. Lichenol. 85: 385 (2002) Literature: Aptroot et al., 2007

998. Platythecium leiogramma (Nyl.) Staiger, Biblioth. Lichenol. 85: 388 (2002) Literature: Aptroot et al., 2007

999. Pliariona montagnei (Bosch) A. Massal., Atti Reale Ist. Veneto Sci. Lett. Arti, Sér. 3, 5: 318 (1860)

Literature: Poengsungnoen et al., 2010 as Thecaria montagnei 
1000. Polyblastidium appendiculatum (Kurok.) Kalb, Phytotaxa 235: 39 (2015)

Literature: Meesim and Mongkolsuk 2010a; Mongkolsuk and Meesim 2012 as Heterodermia appendiculata; Mongkolsuk et al., 2015

1001. Polyblastidium chilense (Kurok.) Kalb, Phytotaxa 235: 40 (2015)

Literature: Meesim and Mongkolsuk 2010a; Mongkolsuk and Meesim 2012 as Heterodermia chilensis

1002. Polybastidium dendriticum (Pers.) Kalb, in Mongkolsuk et al., Phytotaxa 235: $49(2015)$

Literature: Wolseley et al., 2002 as Heterodermia dendritica

1003. Polyblastidium fragilissimum (Kurok.) Kalb, Phytotaxa 235: 41 (2015)

Literature: Mongkolsuk et al., 2015

1004. Polyblastidium hypocaesium (Yasuda) Kalb, Phytotaxa 235(1): 41 (2015)

Literature: Wolseley et al., 2002 as Heterodermia hypocaesia; Mongkolsuk et al., 2015

1005. Polyblastidium hypoleucum (Ach.) Kalb, Phytotaxa 235(1): 42 (2015)

Literature: Vainio 1921 as Anaptychia hypoleuca; Wolseley et al., 2002; Meesim and Mongkolsuk 2010a; Mongkolsuk and Meesim 2012 as Heterodermia hypoleuca; Mongkolsuk et al., 2015

1006. Polyblastidium japonicum (Satô) Kalb, Phytotaxa 235(1): 43 (2015)

Literature: Aptroot et al., 2007; Meesim and Mongkolsuk 2010a; Mongkolsuk and Meesim 2012 as Heterodermia japonica; Mongkolsuk et al., 2015

1007. Polyblastidium microphyllum (Kurok.) Kalb, Phytotaxa 235(1): 44 (2015)

Literature: Boonpragob et al., 1998; Wolseley et al., 2002; Meesim and Mongkolsuk 2010a; Mongkolsuk and Meesim 2012 as Heterodermia microphylla; Mongkolsuk et al., 2015

1008. Polyblastidium propaguliferum (Vain.) Kalb, Phytotaxa 235(1): 45 (2015)

Literature: Schumm and Aptroot 2012 as Heterodermia propagulifera; Mongkolsuk et al., 2015

1009. Polyblastidium queenslandicum (Elix) Kalb, Phytotaxa 235(1): 47 (2015)

Literature: Mongkolsuk et al., 2015

1010. Polyblastidium togashii (Kurok.) Kalb, Phytotaxa 235(1): 47 (2015)

Literature: Mongkolsuk et al., 2015

1011. Polyblastidium violostriatum (Elix) Kalb, Phytotaxa 235(1): 48 (2015)

Literature: Mongkolsuk et al., 2015

1012. Polychidium dendriscum (Nyl.) Henssen, Symb. Bot. Upsal. 18: 107 (1963)

Literature: Vainio 1909 as Leptogidium morreii; Wolseley and Aguirre-Hudson 1995

1013. Polychidium muscicola (Sw.) Gray, Nat. Arr. Brit. Pl. 1: 402 (1820)

Literature: Mongkolsuk et al., 2013

1014. Polychidium stipitatum Vězda \& W.A.Weber, Mycotaxon 3: 355 (1976)

Literature: Aptroot et al., 2007

1015. Polymeridium albidum (Müll.Arg.) R.C.Harris, Acta Amazonica Supl. 14: 69 (1986) 
Literature: Wolseley et al., 2002; Aptroot et al., 2007; Aptroot and Lücking 2016

1016. Polymeridium albocinereum (Kremp.) R.C.Harris, Bol. Mus. Paraense Emílio Goeldi, Ser. Bot. 7: 625 (1993)

Literature: Aptroot and Lücking 2016

1017. Polymeridium catapastum (Nyl.) R.C.Harris., Acta Amazonica 14: 70. (1984)

Literature: Vongshewarat et al., 1999; Wolseley et al., 2002; Aptroot et al., 2007

1018. Polymeridium quinqueseptatum (Nyl.) R.C.Harris in Tucker \& Harris, Bryologist 83: 12. (1980)

Literature: Vongshewarat et al., 1999; Wolseley et al., 2002; Aptroot and Lücking 2016

1019. Polymeridium siamense (Vain.) Aptroot, in Aptroot \& Cáceres, Nova Hedwigia 98: 24 (2014)

Literature: Vainio 1921; Alava 1988 as Arthopyrenia siamensis; Aptroot and Lücking 2016

1020. Porina albicera (Kremp.) Overeem \& D.Overeem, Bull. Jard. Bot. Buitenzorg 4: $112(1922)$

Literature: Boonpragob et al., 1998; Wolseley et al., 2002

1021. Porina americana Fée, Essai Crypt. Écorc. (Paris): 83 (1825) [1824]

Literature: Boonpragob et al., 1998; Wolseley et al., 2002

1022. Porina atriceps (Vain.) Vain., in Lücking \& Vězda, Willdenowia 28 : 189 (1998)

Literature: Papong et al., 2007

1023. Porina atrocoerulea Müll.Arg., Flora $66: 336$ (1883)

Literature: Papong et al., 2007

1024. Porina bellendenica Müll.Arg., Hedwigia 30: 56 (1891)

Literature: Wolseley et al., 2002

1025. Porina borreri (Trevis.) D.Hawksw. \& P.James, in Hawksworth et al., Lichenologist 12: 107 (1980)

Literature: Schumm and Aptroot 2012

1026. Porina conica R.Sant., Symb. Bot. Upsal. 12: 232 (1952)

Literature: Boonpragob et al., 1998; Wolseley et al., 2002

1027. Porina corruscans (Rehm) R.Sant., Symb. Bot. Upsal. 12: 223 (1952)

Literature: Boonpragob et al., 1998; Wolseley et al., 2002

1028. Porina cupreola (Müll.Arg.) F.Schill., Hedwigia 67: 274 (1927)

Literature: Papong et al., 2007

1029. Porina distans Vězda \& Vivant, in Vězda, Nova Hedwigia 58: 136 (1994)

Literature: Aptroot et al., 2007

1030. Porina eminentior (Nyl.) P.M.McCarthy, Lichenologist 32: 42 (2000)

Literature: Wolseley et al., 2002; Aptroot et al., 2007

1031. Porina epiphylla Fée, Essai Crypt. Écorc. (Paris): 76 (1825) [1824]

Literature: Boonpragob et al., 1998; Wolseley et al., 2002

1032. Porina erawanensis P.M.McCarthy \& Vongshew., in Vongshewarat et al., Mycotaxon 70: 231 (1999) 
Literature: Vongshewarat et al., 1999; Wolseley et al., 2002

1033. Porina exocha (Nyl.) P.M.McCarthy, Lichenologist 32: 23 (2000)

Literature: Yoshimura 1978, Homchantara and Coppins 2002 as Myriotrema whalleyanum; Papong et al., 2010

1034. Porina fulvella Müll.Arg., Flora 66: 335 (1883)

Literature: Papong et al., 2007

1035. Porina guentheri (Flot.) Zahlbr., Cat. Lich. Univ. 1: 384 (1922)

Literature: Aptroot et al., 2007

1036. Porina hoehneliana (Jaap) R.Sant., Symb. Bot. Upsal. 12: 262 (1952)

Literature: Papong et al., 2007

1037. Porina huainamdungensis Papong, Thammathaworn \& Lücking, Phytotaxa 18: $100(2011)$

Literature: Lumbsch et al., 2011

1038. Porina internigrans (Nyl.) Müll.Arg., Rep. Australas. Assoc. Advancem. Sci.: $452(1895)$

Literature: Vongshewarat et al., 1999; Wolseley et al., 2002; Schumm and Aptroot 2012

1039. Porina kamerunesis F.Schill., Hedwigia 67: 289 (1927)

Literature: Papong et al., 2007

1040. Porina kansriae P.M.McCarthy, Lichenologist 31: 239 (1999)

Literature: Boonpragob et al., 1998; McCarthy 1999; Wolseley et al., 2002; Aptroot et al., 2007 as Porina ulceratula

1041. Porina karnatakensis Makhija, Adaw. \& Patw., J. Econ. Taxon. Bot. 18: 538 (1995)

Literature: Papong et al., 2007

1042. Porina lucida R.Sant., Symb. Bot. Upsal. 12: 240 (1952)

Literature: Boonpragob et al., 1998; Wolseley et al., 2002

1043. Porina mastoidea Fée, Essai Crypt. Écorc. (Paris): 82 (1825) [1824]

Literature: Wolseley and Aguirre-Hudson 1997a, 1997b; Boonpragob et al., 1998; Wolseley et al., 2002

1044. Porina minutissima Henssen, Lücking \& Vězda, in Lücking \& Vězda, Willdenowia 28: 211 (1998)

Literature: Papong et al., 2007

1045. Porina nitidula Müll.Arg., Flora 66: 336 (1883)

Literature: Aptroot et al., 2007; Papong et al., 2007

1046. Porina palmicola Malcolm \& Vězda, in Vězda, Lichenes Rariores Exsiccati 41: 2 (1999)

Literature: Papong et al., 2007

1047. Porina perminuta Vain., Univ. Calif. Publ. Bot. 12: 14 (1924)

Literature: Papong et al., 2007

1048. Porina rubentior (Stirt.) Müll.Arg., Flora 66: 334 (1883)

Literature: Papong et al., 2007

1049. Porina rufula (Kremp.) Vain., Acta Soc. Fauna Fl. Fenn. 7: 227 (1890) 
Literature: Boonpragob et al., 1998; Wolseley et al., 2002

1050. Porina siamensis P.M.McCarthy, Lichenologist 31: 242 (1999)

Literature: McCarthy 1999; Wolseley et al., 2002

1051. Porina sphaerocephala Vain., Ann. Acad. Sci. Fenn., Ser. A, 15 : 368 (1921)

Literature: Papong et al., 2007

1052. Porina subnucula Lumbsch, Lücking \& Vězda, in Lücking \& Vězda, Willdenowia 28: 217 (1998)

Literature: Papong et al., 2007

1053. Porina subrubrosphaera Kurok., J. Jap. Bot. 33: 206 (1958)

Literature: Papong et al., 2007

1054. Porina terrae-reginae P.M. McCarthy, Lücking \& Vẽzda, Fl. Australia 58: 228 (2001) Literature: Papong et al., 2007 as Porina cupreola

1055. Porina tetracerae (Ach.) Müll.Arg., Bot. Jahrb. Syst. 6: 401 (1885)

Literature: Boonpragob et al., 1998; Wolseley et al., 2002

1056. Porina tetramera (Malme) R.Sant., in Thorold, J. Ecol. 40: 129 (1952)

Literature: Papong et al., 2007

1057. Porina trichothelioides R.Sant., Symb. Bot. Upsal. 12: 227 (1952)

Literature: Aptroot et al., 2007; Papong et al., 2007

1058. Porina vezdae Lücking, Nova Hedwigia 52: 285 (1991)

Literature: Boonpragob et al., 1998; Wolseley et al., 2002

1059. Porina virescens (Kremp.) Müll.Arg., in Lücking \& Vězda, Willdenowia 28: 219 (1998)

Literature: Boonpragob et al., 1998; Wolseley et al., 2002

1060. Porina wolseleyae P.M. McCarthy, Lichenologist 31: 244 (1999)

Literature: McCarthy 1999; Wolseley et al., 2002

1061. Porpidia albocaerulescens (Wulfen) Hertel \& Knoph, in Hertel, Beih. Nova Hedwigia 79: 433 (1984)

Literature: Kalb et al., 2012

1062. Pseudochapsa dilatata (Müll.Arg.) Parnmen, Lücking \& Lumbsch, PLoS ONE 7(12): e51392 (2012)

Literature: Wolseley et al., 2002 as Thelotrema dilatatum

1063. Pseudochapsa phlyctidioides (Müll.Arg.) Parnmen et al., PLoS ONE 7(12): e51392 (2012)

Literature: Boonpragob et al., 1998; Wolseley et al., 2002 as Thelotrema phlyctidioides

1064. Pseudochapsa pseudoexanthismocarpa (Patw. \& C.R. Kulk.) Parnmen, Lücking \& Lumbsch, PLoS ONE 7 (12): e51392 (2012)

Literature: Wolseley et al., 2002 as Thelotrema pseudoexanthismocarpoum

1065. Pseudocyphellaria argyracea (Delise) Vain., Hedwigia 37: 35 (1898)

Literature: Wolseley and Aguirre-Hudson 1995; Boonpragob et al., 1998; Wolseley et al., 2002

1066. Pseudocyphellaria beccarii (Kremp.) D.J.Galloway, Bull. Brit. Mus. (Nat. Hist.) Bot. 24: 119 (1994) 
Literature: Wolseley and Aguirre-Hudson 1997a; Wolseley et al., 2002

1067. Pseudocyphellaria crocata (L.) Vain., Hedwigia 37: 34 (1898)

Literature: Mongkolsuk et al., 2013

1068. Pseudocyphellaria junghuhniana (Müll.Arg.) D.D.Awasthi, Beih. Nova Hedwigia 17: 104 (1965)

Literature: Wolseley and Aguirre-Hudson 1995; Aptroot et al., 2007

1069. Pseudopyrenula diluta (Fée) Müll.Arg., Flora 66: 249 (1883)

Literature: Vainio 1909; Aptroot et al., 2007; Aptroot and Lücking 2016

1070. Pseudopyrenula endoxanthoides Vain., Hedwigia 46: 180 (1907)

Literature: Alava 1988; Aptroot and Lücking 2016

1071. Pseudopyrenula subnudata Müll.Arg., Flora 66: 272 (1883)

Literature: Wolseley et al., 2002 as Pseudopyrenula diluta var. degenerans; Aptroot and Lücking 2016

1072. Pseudotopeliopsis laceratula (Müll.Arg.) Parnmen, Lücking \& Lumbsch, PLoS ONE 7 (12): e51392 (2012)

Literature: Wolseley et al., 2002 as Thelotrema laceratulum; Parnmen et al., 2012; Lumbsch et al., 2014; Poengsungnoen et al., 2014b

1073. Psoroglaena cubensis Müll.Arg., Flora 74: 381 (1891)

Literature: Aptroot et al., 2007

1074. Psorotheciopsis patellarioides (Rehm) R.Sant., Symb. Bot. Upsal. 12: 336 (1952) Literature: Aptroot et al., 2007

1075. Punctonora nigropulvinata Aptroot, in Aptroot et al., Biblioth. Lichenol. 64: 151 (1997)

Literature: Aptroot et al., 2007

1076. Pyrenula acutalis R.C.Harris, Mem. New York Bot. Gard. 49: 85 (1989)

Literature: Aptroot et al., 2007

1077. Pyrenula anomala (Ach.) Vain., Ann. Acad. Sci. Fenn., Ser. A, 6: 189 (1915)

Literature: Wolseley et al., 2002

1078. Pyrenula aspistea (Ach.) Ach., Syn. Meth. Lich. 123 (1814)

Literature: Vainio 1909; Paulson 1930; Wolseley et al., 2002 as Pyrenula subaggregata and Pyrenula velatior; Gueidan et al., 2016

1079. Pyrenula aurantiopileata Aptroot, in Aptroot et al., Fungal Diversity 24: 118 (2007) Literature: Aptroot et al., 2007

1080. Pyrenula breutelii (Müll. Arg.) Aptroot, Lichenologist 44(1): 35 (2012)

Literature: Vongshewarat et al., 1999; Wolseley et al., 2002; Aptroot et al., 2007 as Pyrenula macularis

1081. Pyrenula castanea (Eschw.) Müll. Arg., Flora, Regensburg 67(35): 664 (1884) Literature: Vainio 1909; Alava 1988; Wolseley et al., 2002 as Pyrenula submarginata

1082. Pyrenula circumfiniens Vain., Ann. Acad. Sci. fenn., Ser. A 6(no. 7): 195 (1915) Literature: Vongshewarat et al., 1999; Wolseley et al., 2002; Aptroot et al., 2007 as Pyrenula subferruginea

1083. Pyrenula confinis (Nyl.) R.C.Harris, More Florida Lichens, Incl. 10 Cent Tour Pyrenol. (New York): 109 (1995) 
Literature: Vainio 1909 as Bottaria confinis; Wolseley et al., 2002

1084. Pyrenula cuyabensis (Malme) R.C.Harris, Mem. New York Bot. Gard. 49: 91 (1989)

Literature: Aptroot et al., 2007

1085. Pyrenula fetivica (Kremp.) Müll. Arg., Flora, Regensburg 72: 68 (1889)

Literature: Vainio 1909; Alava 1988; Wolseley et al., 2002 as Pyrenula feracissima, Pyrenula approximata and Pyrenula glabriuscula

1086. Pyrenula globifera (Eschw.) Aptroot, in Aptroot, Lücking, Sipman, Umaña \& Chaves, Biblioth. Lichenol. 97: 103 (2008)

Literature: Aptroot et al., 2007 as Anthracothecium globiferum

1087. Pyrenula immissa (Stirt.) Zahlbr., Cat. Lich. Univers. 1: 433 (1922)

Literature: Aptroot et al., 2007 as Pyrenula laii

1088. Pyrenula leucostoma Ach., Syn. Meth. Lich.: 124 (1814)

Literature: Vainio 1909 as Bottaria libricola; Wolseley et al., 2002

1089. Pyrenula lineatostroma Aptroot, in Aptroot et al., Biblioth. Lichenol. 64: 160 (1997)

Literature: Aptroot et al., 2007

1090. Pyrenula mamillana (Ach.) Trevis., Consp. Verruc.: 13 (1860)

Literature: Aptroot et al., 2007; Schumm and Aptroot 2012

1091. Pyrenula massariospora (Starbäck) R.C.Harris, Mem. New York Bot. Gard. 49: 95 (1989)

Literature: Aptroot et al., 2007

1092. Pyrenula microcarpa Müll.Arg., Bot. Jahrb. Syst.6: 412 (1885)

Literature: Aptroot et al., 2007

1093. Pyrenula montana Aptroot, in Aptroot et al., Biblioth. Lichenol. 64: 163 (1997)

Literature: Aptroot et al., 2007

1094. Pyrenula nitida (Weigel) Ach., Syn. Meth. Lich.: 125 (1814)

Literature: Paulson 1930; Wolseley et al., 2002

1095. Pyrenula ochraceoflava var. pacifica P.M. McCarthy, Lichenologist 32: 32 (2000)

Literature: Vainio 1907; Vainio 1909; Alava 1988 as Bottaria rosea; Wolseley et al., 2002 as Anthracothecium roseum

1096. Pyrenula oleosa R.C.Harris, Mem. New York Bot. Gard. 49: 97 (1989)

Literature: Aptroot et al., 2007

1097. Pyrenula parvinuclea (Meyen \& Flot.) Aptroot, in Aptroot et al., Biblioth. Lichenol. 64: 165 (1997)

Literature: Vainio 1907; Vainio 1909 as Bottaria denudata and Anthracothecium parameroides; Alava 1988 as Bottaria parameroides; Vongshewarat et al., 1999;

Wolseley et al., 2002 as Anthracothecium parameroides; Gueidan et al., 2016

1098. Pyrenula quassiicola Fée, Essai Crypt. Écorc., Suppl. Révis. (Paris): 79 (1837)

Literature: Aptroot et al., 2007

1099. Pyrenula rockii Zahlbr., Ann. Mycol., 30: 428 (1932) 
Literature: Aptroot et al., 2007

1100. Pyrenula rubrostoma R.C.Harris, in Tucker \& Harris, Bryologist 83: 16 (1980) Literature: Aptroot et al., 2007

1101. Pyrenula santensis (Nyl.) Müll.Arg., Flora 65: 400 (1882)

Literature: Aptroot et al., 2007

1102. Pyrenula scutata (Stirt.) Zahlbr., Cat. Lich. Univers. 1: 452 (1922)

Literature: Aptroot et al., 2007 as Pyrenula pileata

1103. Pyrenula septicollaris (Eschw.) R.C.Harris, Mem. New York Bot. Gard. 49: 101 (1989) Literature: Schumm and Aptroot 2012

1104. Pyrenula sexlocularis (Nyl.) Müll. Arg., Flora, Regensburg 60: 475 (1879) Literature: Gueidan et al., 2016; Aptroot et al., 2007 as Pyrenula concatervans

1105. Pyrenula thailandica Aptroot, Lichenologist 44: 617 (2012) Literature: Aptroot et al., 2012; Schumm and Aptroot 2012

1106. Pyrgidium montellicum (Beltr.) Tibell, Lichenologist 14: 239 (1982) Literature: Aptroot et al., 2007

1107. Pyrgillus indicus (Kremp.) Aptroot, Biblioth. Lichenol. 44: 84 (1991) Literature: Wolseley and Aguirre-Hudson 1997a; 1997b as Pyrgillocarpon indicum; Wolseley et al., 2002

1108. Pyrgillus javanicus (Mont. \& Bosch) Nyl., Mém. Soc. Sci. Nat. Math. Cherbourgg 5: 334 (1858)

Literature: Wolseley and Aguirre-Hudson 1997a, 1997b; Boonpragob et al., 1998; Wolseley et al., 2002

1109. Pyrrhospora fuscisidiata Aptroot \& Wolseley, in Aptroot et al., Fungal Diversity 24: 121 (2007)

Literature: Aptroot et al., 2007

1110. Pyrrhospora luminescens Aptroot \& Wolseley, in Aptroot et al., Fungal Diversity 24: 122 (2007)

Literature: Aptroot et al., 2007

1111. Pyxine asiatica Vain., Hedwigia, 46: 171 (1907)

Literature: Alava 1988; Mongkolsuk et al., 2012; Van den Boom et al., 2014

1112. Pyxine australiensis Kalb, Herzogia10: 61 (1994)

Literature: Mongkolsuk et al., 2012

1113. Pyxine berteriana (Fée) Imshaug, Trans. Amer. Microscop. Soc. 56: 254 (1957) Literature: Meesim and Mongkolsuk 2010a; Mongkolsuk et al., 2012; Mongkolsuk and Meesim 2012; Schumm and Aptroot 2012; Van den Boom et al., 2014

1114. Pyxine boonpragobiana Kalb \& Mongkolsuk, Phytotaxa 59: 38 (2012) Literature: Mongkolsuk et al., 2012

1115. Pyxine coccifera (Fée) Nyl., Mém. Soc. Sci. Nat. Cherbourg 5: 108 (1857) Literature: Vainio 1921; Wolseley and Aguirre-Hudson 1995; 1997a; 1997b; Wolseley et al., 1994; Wolseley et al., 2002; Meesim and Mongkolsuk 2010a; Mongkolsuk et al., 2012; Mongkolsuk and Meesim 2012; Van den Boom et al., 2014

1116. Pyxine cocoes (Sw.) Nyl., Mém. Soc. Sci. Nat. Cherbourg 5: 108 (1857) 
Literature: Saipunkaew et al., 2007; Meesim and Mongkolsuk 2010a; Mongkolsuk et al., 2012; Mongkolsuk and Meesim 2012; Schumm and Aptroot 2012

1117. Pyxine cognata Stirt., Proc. Roy. Philos. Soc. Glasgow 11:311 (1879) [1878] Literature: Mongkolsuk et al., 2012

1118. Pyxine copelandii Vain., Philipp. J. Sci., C 8: 110 (1913)

Literature: Vainio 1921

1119. Pyxine coralligera Malme, Bih. Kongl. Svenska Vetensk.-Akad. Handl. 23: 40 (1897)

Literature: Wolseley and Aguirre-Hudson 1997a; 1997b; Wolseley et al., 2002; Aptroot et al., 2007; Mongkolsuk et al., 2012; Mongkolsuk and Meesim 2012

1120. Pyxine cylindrica Kashiw., Bull. Natl. Sci. Mus., Tokyo, B. 3: 66 (1977) Literature: Aptroot et al., 2007; Mongkolsuk et al., 2012

1121. Pyxine dactyloschmidtii Kalb \& Mongkolsuk, Phytotaxa 59: 44 (2012) Literature: Mongkolsuk et al., 2012

1122. Pyxine daedalea Krog \& R.Sant., in Moberg, Thunbergia 2: 7 (1986) Literature: Aptroot et al., 2007; Mongkolsuk et al., 2012

1123. Pyxine farinosa Kashiw., Bull. Natl. Sci. Mus., Tokyo, B. 3: 67 (1977) Literature: Aptroot et al., 2007; Mongkolsuk et al., 2012; Van den Boom et al., 2014

1124. Pyxine katendei Swinscow \& Krog, Norweg. J. Bot. 22: 54 (1975) Literature: Meesim and Mongkolsuk 2010a; Mongkolsuk and Meesim 2012

1125. Pyxine meissneriana Nyl., Bull. Soc. Linn. Normandie 2:164 (1873)

Literature: Meesim and Mongkolsuk 2010a; Mongkolsuk et al., 2012; Mongkolsuk and Meesim 2012; Van den Boom et al., 2014

1126. Pyxine obscurascens Malme, Bih. Kongl.. Svenska Vetensk.-Akad. Handl. 23:42 (1897)

Literature: Aptroot et al., 2007

1127. Pyxine petricola Nyl., in Crombie, J. Bot., Lond. 14: 263 (1876)

Literature: Aptroot et al., 2007; Mongkolsuk et al., 2012; Van den Boom et al., 2014

1128. Pyxine philippina Vain., Philipp. J. Sci., C 8: 110 (1913)

Literature: Mongkolsuk et al., 2012

1129. Pyxine profallax Kalb, in Kalb et al., Biblioth. Lichenol.99: 243 (2009)

Literature: Mongkolsuk et al., 2012

1130. Pyxine pseudokeralensis Kalb, Mongkolsuk \& Buaruang, Phytotaxa 59: 49 (2012)

Literature: Mongkolsuk et al., 2012

1131. Pyxine pyxinoides (Müll.Arg.) Kalb, Biblioth. Lichenol. 24: 66 (1987)

Literature: Meesim and Mongkolsuk 2010a as Pyxine microspore; Mongkolsuk and Meesim 2012

1132. Pyxine reticulata (Vain.) Vain., Ann. Acad. Sci. Fenn., Ser. A. 6: 70 (1914). Literature: Meesim and Mongkolsuk 2010a; Mongkolsuk and Meesim 2012 
1133. Pyxine retirugella Nyl., Ann. Sci. Nat., Bot. 11: 240 (1859)

Literature: Vainio 1909; Alava 1988 as Pyxine asiatica and Pyxine retirugella var. laevior; Wolseley and Aguirre-Hudson 1995; 1997a; 1997b; Wolseley et al., 2002; Saipunkaew et al., 2007; Meesim and Mongkolsuk 2010a; Mongkolsuk et al., 2012; Schumm and Aptroot 2012; Mongkolsuk and Meesim 2012 as Pyxine consocians

1134. Pyxine schmidtii Vain., Hedwigia 46: 170 (1907)

Literature: Vainio 1909; Alava 1988; Wolseley et al., 2002; Mongkolsuk et al., 2012

1135. Pyxine sorediata (Ach.) Mont. in Sagva. Hist. Phys. Cuba, Bot., Pl. Cell. 9:188 (1842)

Literature: Aptroot et al., 2007; Meesim and Mongkolsuk 2010a; Mongkolsuk et al., 2012; Mongkolsuk and Meesim 2012

1136. Pyxine subcinerea Stirton, Trans. \& Proc. New Zealand Inst. 30: 397 (1898)

Literature: Aptroot et al., 2007; Meesim and Mongkolsuk 2010a; Mongkolsuk et al., 2012; Mongkolsuk and Meesim 2012

1137. Pyxine subcoralligera Kalb, Mongk. \& Boonpr., in Mongkolsuk, Meesim, Poengsungnoen \& Kalb, Phytotaxa 59: 52 (2012)

Literature: Mongkolsuk et al., 2012

1138. Pyxine vermiformis Swinscow \& Krog, Norweg. J. Bot. 22: 66 (1975)

Literature: Meesim and Mongkolsuk 2010a; Mongkolsuk and Meesim 2012

1139. Ramalina calicaris (L.) Röhl., Deutschl. Fl. 3: 139 (1813)

Literature: Satô 1962; Yoshimura 1978; Wolseley et al., 2002

1140. Ramalina conduplicans Vain., Suom. Elain-ja Kasvit. Seuran Van. Julk. 1: 35 (1921)

Literature: Satô 1962; Vainio 1921; Wolseley et al., 2002

1141. Ramalina farinacea (L.) Ach., Lichenogr. Universalis: 606 (1810)

Literature: Paulson 1930; Vainio 1909; Yoshimura 1978; Wolseley et al., 2002

1142. Ramalina hossei Vain., Ann. Bot. Soc. Zool.-Bot. Fenn. "Vanamo" 1: 36 (1921) Literature: Wolseley et al., 2002

1143. Ramboldia russula (Ach.) Kalb, Lumbsch \& Elix, Nova Hedwigia 86: 37 (2008) Literature: Satô 1962; Vainio 1921 as Lecidea russula; Wolseley and AguirreHudson 1997; Boonpragob et al., 1998; Wolseley et al., 2002 as Pyrrhospora russula; Kalb et al., 2009b; Schumm and Aptroot 2012

1144. Ramboldia siamensis Buaruang, Elix \& Kalb, Mycotaxon 110: 120 (2009)

Literature: Kalb et al., 2009b

1145. Relicina abstrusa (Vain.) Hale, Phytologia 28: 484 (1974)

Literature: Boonpragob et al., 1998; Moon et al., 2000a; Wolseley et al., 2002; Buaruang et al., 2009; Buaruang et al., 2015

1146. Relicina circumnodata (Nyl.) Hale, Phytologia 28: 484 (1974)

Literature: Moon et al., 2000a; Wolseley et al., 2002

1147. Relicina intertexta (Mont. \& Bosch) Kirika, Divakar \& Lumbsch, Lichenologist 49: 485 (2017) 
Literature: Vainio 1909 as Parmelia ecoronata; Hale 1976c as Pseudoparmelia intertexta; Wolseley et al., 2002; Buaruang et al., 2009; Buaruang et al., 2015 as Relicinopsis intertexta; Kirika et al., 2017

1148. Relicina malaccensis (Nyl.) Kirika, Divakar \& Lumbsch, Lichenologist 49: 485 (2017)

Literature: Pooprang et al., 1999; Wolseley et al., 2002; Louwhoff et al., 2012; Buaruang et al., 2015 as Relicinopsis malaccensis; Kirika et al., 2017

1149. Relicina palmata Elix, Biblioth. Lichenol. 62: 83 (1996)

Literature: Noicharoen et al., 2003

1150. Relicina planiuscula (Kurok.) Hale, Phytologia 28: 484 (1974)

Literature: Aptroot et al., 2007; Louwhoff et al., 2012

1151. Relicina polycarpa Elix \& Polyiam, in Noicharoen et al., Mycotaxon 85: 328 (2003)

Literature: Noicharoen et al., 2003

1152. Relicina rahengensis (Vain.) Kirika, Divakar \& Lumbsch, Lichenologist 49: 485 (2017)

Literature: Hale 1976c; Vainio 1921 as Pseudoparmelia rahengensis; Wolseley and Aguirre-Hudson 1995; 1997a; Moon et al., 2000a; Louwhoff et al., 2012; Buaruang et al., 2015 as Relicinopsis rahengensis; Kirika et al., 2017

1153. Relicina subabstrusa (Gyeln.) Hale, Phytologia 28: 485 (1974)

Literature: Boonpragob et al., 1998; Wolseley et al., 2002; Buaruang et al., 2015

1154. Relicina subconnivens Hale, Smithsonian Contr. Bot. 26: 30 (1975)

Literature: Noicharoen et al., 2003

1155. Relicina sublanea (Kurok.) Hale, Phytologia 28: 485 (1974)

Literature: Wolseley et al., 2002

1156. Relicina sublimbata (Nyl.) Hale, Phytologia 28: 485 (1974)

Literature: Hale 1975; Wolseley and Aguirre-Hudson 1995 as Relicina sublimate; Moon et al., 2000a; Wolseley et al., 2002

1157. Remototrachyna adducta (Nyl.) A.Crespo et al., Taxon 97: 586 (2010)

Literature: Pooprang et al., 1999; Moon et al., 2000a; Wolseley et al., 2002; Buaruang et al., 2009; Louwhoff et al., 2012 as Hypotrachyna adducta

1158. Remototrachyna awasthii (Hale \& Patw.) Divakar \& A.Crespo, Amer. J. Bot. 97: 586 (2010)

Literature: Louwhoff et al., 2012

1159. Remototrachyna crenata (Kurok.) Divakar \& A.Crespo, Taxon 97: 586 (2010) Literature: Moon et al., 2000a as Hypotrachyna crenata

1160. Remototrachyna kingii (Hale) Divakar \& A.Crespo, Amer. J. Bot. 97: 586 (2010)

Literature: Hale 1968 as Parmelia kingii; Moon et al., 2000a; Wolseley et al., 2002; Buaruang et al., 2009; Louwhoff et al., 2012 as Hypotrachyna kingii

1161. Rhabdodiscus asiaticus (Vain.) Rivas Plata, Lücking \& Lumbsch, Taxon 61: 1175 (2012) 
Literature: Vainio 1909; Alava 1988 as Thelotrema asiatica; Boonpragob et al., 1998; Wolseley et al., 2002 as Ocellularia asiatica; Sutjaritturakan and Kalb 2015

1162. Rhabdodiscus crassus (Müll.Arg.) Rivas Plata, Lücking \& Lumbsch, Taxon 6: 1175 (2013)

Literature: Wolseley et al., 2002 as Ocellularia crassa; Sutjaritturakan and Kalb 2015

1163. Rhabdodiscus emersus (Kremp.) Rivas Plata, Lücking \& Lumbsch, Taxon 6: 1175 (2013)

Literature: Boonpragob et al., 1998; Wolseley et al., 2002 as Ocellularia emersa; Sutjaritturakan and Kalb 2015

1164. Rhabdodiscus epitrypus (Nyl.) Vain., Ann. Acad. Sci. Fenn., Ser. A, 15: 184 (1921)

Literature: Wolseley et al., 2002 as Ocellularia epitrypa; Sutjaritturakan and Kalb 2015

1165. Rhabdodiscus fissus (Nyl.) Vain., Ann. Acad. Sci. Fenn., Ser. A, 15: 184 (1921) Literature: Boonpragob et al., 1998; Wolseley et al., 2002 as Ocellularia fissa

1166. Rhabdodiscus marivelensis (Vain.) Rivas Plata, Lücking \& Lumbsch, Taxon 6: 1176 (2013)

Literature: Boonpragob et al., 1998; Kraichak et al. 2014; Wolseley et al., 2002 as Ocellularia marivelensis; Sutjaritturakan and Kalb 2015; Lücking et al., 2015

1167. Rhabdodiscus subcavatus (Nyl.) Rivas Plata \& Lumbsch, in Rivas Plata et al., Taxon 61: 1176 (2012)

Literature: Papong et al., 2014

1168. Ricasolia discolor (Bory) Nyl., Mém. Soc. Imp. Sci. Nat. Cherbourg 3: 174 (1855)

Literature: Yoshimura 1978; Wolseley et al., 2002 as Lobaria discolor

1169. Rinodina citrinisidiata Aptroot \& Wolseley, in Aptroot et al., Fungal Diversity 24: 124 (2007)

Literature: Aptroot et al., 2007

1170. Rinodina colorans Vain ., Acta Soc. Fauna Fl. Fenn. 7: 159 (1890)

Literature: Van den Boom et al., 2014

1171. Rinodina guianensis Aptroot, Proc. Kon. Ned. Akad. Wetensch., Biol. Chem. Geol. Phys. Med. Sci. 90: 240 (1997)

Literature: Van den Boom et al., 2014

1172. Rinodina neglecta Aptroot, Proc. Kon. Ned. Akad. Wetensch., Biol. Chem. Geol. Phys. Med. Sci. 90: 240 (1997)

Literature: Aptroot et al., 2007; Schumm and Aptroot 2012

1173. Rinodina oxydata (A.Massal.) A.Massal., Geneac. Lich.: 19 (1854)

Literature: Aptroot et al., 2007

1174. Rinodina thiomela (Nyl.) Müll.Arg., Flora 64: 515 (1881)

Literature: Aptroot et al., 2007 
1175. Roccella montagnei Bel., Voy. Indes Or. 2: 117 (1834)

Literature: Paulson 1930; Wolseley and Aguirre-Hudson 1995; Wolseley et al., 2002 as Roccella phycopsis

1176. Rolfidium coccocarpioides (Nyl.) Timdal, Opera Bot. 110: 121 (1991)

1177. Rolueckia siamensis Papong, Thammathaworn \& Boonpragob, Nova Hedwigia 86: 205 (2008)

Literature: Papong et al., 2008

1178. Rostania coccophylla (Nyl.) Otálora, P.M.Jørg. \& Wedin, Fungal Diversity 64: 289 (2014)

Literature: Wolseley et al., 2002 as Collema coccophyllum

1179. Sanguinotrema wightii (Taylor) Lücking, in Lücking, Mangold, Plata, Parnmen, Kraichak \& Lumbsch, J. Linn. Soc., Bot.: 441 (2015)

Literature: Wolseley et al., 2002 as Myriotrema wightii; Lücking et al., 2015 as Leptotrema wightii

1180. Sarcographa cinchonarum Fée, Essai Crypt. Écorc. 90: 58 (1824)

Literature: Aptroot et al., 2007

1181. Sarcographa glyphiza (Nyl.) Kr.P.Singh \& G.P. Sinha, Indian Lichens: 404 (2010)

Literature: Aptroot et al., 2007; Poengsungnoen et al., 2010 as Sarcographina glyphiza

1182. Sarcographa heteroclita (Mont.) Zahlbr., in Rechinger, Denkschr. Kaiserl. Akad. Wiss., Wien. Math.-Naturwiss. Kl. 88: 19 (1911)

Literature: Aptroot et al., 2007

1183. Sarcographa labyrinthica (Ach.) Müll.Arg., Mém. Soc. Phys. Genève 29: 62 (1887)

Literature: Vainio 1909 as Graphis labyrinthica; Wolseley et al., 2002; Aptroot et al., 2007; Poengsungnoen et al., 2010

1184. Sarcographa medusulina (Nyl.) Müll.Arg., Flora 70: 77 (1887)

Literature: Wolseley and Aguirre-Hudson 1997a; 1997b; Boonpragob et al., 1998; Wolseley et al., 2002

1185. Sarcographa tricosa (Ach.) Müll.Arg., Mém. Soc. Phys. Genève 29: 63 (1887)

Literature: Aptroot et al., 2007

1186. Sarcographa verrucosa (Vain.) Zahlbr., Cat. Lich. Univ. 2: 467 (1923) [1924]

Literature: Poengsungnoen et al., 2010

1187. Schistophoron tenue Stirt., Rep. Trans. Glasgow Soc. Field Naturalists 4: 165 (1876)

Literature: Aptroot et al., 2007

1188. Scoliciosporum umbrinum (Ach.) Arnold, Flora 54: 50 (1871)

Literature: Paulson 1930; Wolseley et al., 2002 as Bacidia umbrina var. turgida

1189. Sculptolumina japonica (Tuck.) Marbach. Biblioth. Lichenol. 74: 296 (2000)

Literature: Aptroot et al., 2007; Van den Boom et al., 2014

1190. Septotrapelia triseptata (Hepp) Aptroot,in Aptroot et al., Fungal Diversity 24: 122 (2007) 
Literature: Aptroot et al., 2007

1191. Septotrapelia usnica (Sipman) Kalb \& Bungartz, Phytotaxa 150(1): 11 (2013) Literature: Aptroot et al., 2007 as Lepraria usnica

1192. Spilonema schmidtii (Vain.) Henssen., Symb, Bot. Upsal. 18: (1963)

Literature: Wolseley et al., 2002

1193. Sporopodium argillaceum (Müll.Arg.) Zahlbr., Cat. Lich. Univ. 2: 679 (1924) Literature: Papong et al., 2007

1194. Sporopodium flavescens (R.Sant.) Vězda, Lichenes Selecti Exsiccati, Fascicle 5: 2193 (1988)

Literature: Aptroot et al., 2007

1195. Sporopodium hossei Vain., Suom. Elain-ja Kasvit. Seuran Van. Julk. 1: 47 (1921)

Literature: Vainio 1921; Alava 1988; Wolseley et al., 2002

1196. Sporopodium leprieurii Mont., Ann. Sci. Nat., Bot. 16: 54 (1851)

Literature: Aptroot et al., 2007

1197. Sporopodium leucoxanthum (Spreng.) Vain., Suom. Elain-ja Kasvit. Seuran Van. Julk. 1: 47 (1921)

Literature: Vainio 1921; Wolseley et al., 2002

1198. Sporopodium phyllocharis (Mont.) A.Massal., Alc. Gen. Lich. 21: 98 (1997) Literature: Papong et al., 2007

1199. Sporopodium xantholeucum (Müll.Arg.) Zahlbr., Cat. Lich. Univ. 2: 681 (1924) Literature: Aptroot et al., 2007

1200. Stegobolus berkeleyanus Mont., in Hooker, London J. Bot. 4: 4 (1845) Literature: Kraichak et al. 2014; Papong et al., 2014; Lücking et al., 2015

1201. Sticta boschiana Mont. \& Bosch., Syll. Gen. Sp. Crypt.: 327 (1856) Literature: Wolseley et al., 2002

1202. Sticta fuliginosa (Hoffm.) Ach., Methodus (Acharius): 280 (1803) Literature: Wolseley et al., 2002

1203. Sticta weigelii Isert, in Acharius, Lichenogr. Universalis 446 (1810) Literature: Yoshimura 1978; Wolseley et al., 2002

1204. Stigmatochroma epimarta (Nyl.) Marbach, Biblioth. Lichenol. 74: 311 (2000) Literature: Aptroot et al., 2007; Van den Boom et al., 2014

1205. Stigmatochroma gerontoides (Stirt.) Marbach, Biblioth. Lichenol. 74: 314 (2000) Literature: Van den Boom et al., 2014

1206. Stigmatochroma metaleptodes (Nyl.) Marbach, Biblioth. Lichenol. 74: 319 (2000)

Literature: Aptroot et al., 2007

1207. Stirtonia rhizophorae Kalb \& Mongkolsuk, in Kalb et al., Phytotaxa 42: 45 (2012)

Literature: Kalb et al., 2012; Schumm and Aptroot 2012

1208. Strigula antillarum (Fée) R.Sant., Bot. Jahrb. Syst. 6: 379 (1885)

Literature: Aptroot et al., 2007

1209. Strigula concreta (Fée) R.Sant., Symb. Bot. Upsal. 12: 177 (1952) 
Literature: Aptroot et al., 2007; Papong et al., 2007

1210. Strigula maculata (Cooke \& Massee) R.Sant., Symb. Bot. Upsal. 12: 186 (1952) Literature: Boonpragob et al., 1998; Wolseley et al., 2002

1211. Strigula melanobapha (Kremp.) R.Sant., Symb. Bot. Upsal. 12: 188 (1952)

Literature: Boonpragob et al., 1998; Wolseley et al., 2002

1212. Strigula nemathora Mont., Hist. Phys. Cuba, Bot., Pl. Cell. 2: 139 (1842)

Literature: Aptroot et al., 2007

1213. Strigula nitidula Mont. in Sagra, Hist. Phys. Cuba, Bot., Pl. Cell. 9 : 184 (1842) Literature: Aptroot et al., 2007; Papong et al., 2007

1214. Strigula obducta (Müll.Arg.) R.C.Harris, More Florida Lichens, Incl. 10 Cent Tour Pyrenol. (New York): 158 (1995)

Literature: Papong et al., 2007

1215. Strigula phaea (Ach.) R.C.Harris, in Tucker \& Harris, Bryologist 83: 18 (1980) Literature: Aptroot et al., 2007

1216. Strigula phyllogena (Müll.Arg.) R.C.Harris, More Florida Lichens, Incl. 10 Cent Tour Pyrenol. (New York): 159 (1995)

Literature: Papong et al., 2007

1217. Strigula smaragdula Fr., Linnaea 5: 550 (1830)

Literature: Boonpragob et al., 1998; Wolseley et al., 2002

1218. Strigula subtilissima (Fée) Müll.Arg., Flora 66: 346 (1883)

Literature: Boonpragob et al., 1998; Wolseley et al., 2002; Papong et al., 2007

1219. Syncesia albiseda (Nyl.) Tehler, Fl. Neotrop. Monogr. 78: 37 (1996)

Literature: Wolseley et al., 2002; Wolseley and Aguirre-Hudson 1997b

1220. Tapellaria nigrata (Müll.Arg.) R.Sant., Symb. Bot. Upsal. 12: 499 (1952)

Literature: Papong et al., 2007

1221. Telochistes flavicans (Sw.) Norman, Nytt Mag. Naturvidensk. 7: 229 (1853)

Literature: Vainio 1909; Wolseley and Aguirre-Hudson 1995; Wolseley et al., 2002

1222. Tephromela atra (Huds.) Hafeller, in Kalb, Lich. Neotrop. Exsicc., 8: 297 (1983)

Literature: Aptroot et al., 2007

1223. Tephromela bunyana Kalb \& Elix, in Kalb, Biblioth. Lichenol. 88: 319 (2004) Literature: Kalb et al., 2009

1224. Tephromela physodica Kalb, in Türk, John \& Hauck (eds), Sauteria 15: 245 (2008)

Literature: Kalb et al., 2009

1225. Thamnolia vermicularis (Sw.) Schaer., Enum. Crit. Lich. Eur.: 243 (1850)

Literature: Satô 1962

1226. Thecaria quassiicola Fée, Essai Crypt. Écorc. (Paris): 92 (1825) [1824]

Literature: Aptroot et al., 2007; Poengsungnoen et al., 2010

1227. Thelotrema adjectum Nyl., Flora 49: 290 (1866)

Literature: Parnmen et al., 2012

1228. Thelotrema canarense Patw. \& C.R.Kulk., Norweg. J. Bot. 24: 128 (1977) 
Literature: Wolseley et al., 2002

1229. Thelotrema defossum (Müll.Arg.) Mangold, Aust. Syst. Bot. 21: 221 (2008)

Literature: Schumm and Aptroot 2012; Papong et al., 2014

1230. Thelotrema lacteum Kremp., in Nylander, Flora 47: 269 (1864)

Literature: Wolseley et al., 2002

1231. Thelotrema lepademersum Nagarkar, Sethy \& Patw., Kavaka 12: 59 (1985)

Literature: Wolseley et al., 2002

1232. Thelotrema mongkolsukii Homchant. \& Coppins, Lichenologist 34: 130 (2002)

Literature: Yoshimura 1978; Homchantara and Coppins 2002; Wolseley et al., 2002; Papong et al., 2010

1233. Thelotrema monosporoides Nyl., Lich. Nov. Zeland. (Paris): 76 (1888)

Literature: Homchantara 1999; Wolseley et al., 2002 as Ocellularia monosporoides

1234. Thelotrema monosporum Nyl., Ann. Sci. Nat., Bot. 15: 46 (1860)

Literature: Wolseley et al., 2002

1235. Thelotrema nureliyum Hale, Bull. Brit. Mus. (Nat. Hist.), Bot. 8: 261 (1981)

Literature: Boonpragob et al., 1998; Wolseley et al., 2002; Parnmen et al., 2012 as Thelotrema cf. nureliyum

1236. Thelotrema patwardhanii (Hale) Rivas Plata \& Mangold, in Rivas Plata et al., Lichenologist 42: 184 (2010)

Literature: Yoshimura 1978; Homchantara and Coppins 2002 and Wolseley et al., 2002 as Myriothema grandissporum; Papong et al., 2010

1237. Thelotrema poeltii Patw. \& C.R.Kulk., Norweg. J. Bot. 24: 130 (1977)

Literature: Boonpragob et al., 1998; Wolseley et al., 2002

1238. Thelotrema porinoides Mont. \& Bosch, Syll. Gen. Sp. Crypt.: 151 (1855)

Literature: Boonpragob et al., 1998; Wolseley et al., 2002

1239. Thelotrema rugatulum Nyl., Bull. Soc. Linn. Normandie 7: 168 (1873)

Literature: Boonpragob et al., 1998; Wolseley et al., 2002

1240. Thelotrema subadjectum Mangold, Fl. Australia 57: 658 (2009)

Literature: Papong et al., 2014

1241. Thelotrema turgidulum (Müll.Arg.) Hale, Mycotaxon 11: 132 (1980)

Literature: Boonpragob et al., 1998; Wolseley et al., 2002

1242. Thelotrema weberi Hale, Phytologia 27: 497 (1974)

Literature: Wolseley et al., 2002

1243. Tomasellia eschweileri (Müll.Arg.) R.C.Harris, in Tucker \& Harris, Bryologist 83: 19 (1980)

Literature: Aptroot et al., 2007

1244. Topeliopsis darlingtonii Frisch \& Kalb, Lichenologist 38: 39 (2006)

Literature: Aptroot et al., 2007

1245. Trapelia coarctata (Turner ex Sm.) M.Choisy, in Werner, Bull. Soc. Sci. Nat. Phys. Maroc 12: 160 (1932)

Literature: Aptroot et al., 2007

1246. Trapelia placodioides Coppins \& P. James, Lichenologist 16(3): 257 (1984) 
1247. Trapeliopsis viridescens (Schard.) Coppins \& P.James, Lichenologist 16: 263 (1984)

Literature: Aptroot et al., 2007

1248. Traponora asterella Aptroot, in Diederich et al., Biblioth. Lichenol. 64: 199 (1997) Literature: Aptroot et al., 2007; 2009

1249. Traponora globosa Aptroot, Biblioth. Lichenol. 100: 24 (2009)

Literature: Aptroot 2009

1250. Tricharia demoulinii Sérus, in Aptroot et al., Biblioth. Lichenol. 64: 200 (1997) Literature: Papong et al., 2007

1251. Tricharia vainioi R.Sant., Symb. Bot. Upsal. 12: 382 (1952)

Literature: Boonpragob et al., 1998; Wolseley et al., 2002

1252. Trichothelium ake-assii Becker \& Lücking, in Daniels et al., Flechten Follmann: 170 (1995)

Literature: Papong et al., 2007

1253. Trichothelium alboatrum Vain., Ann. Acad. Sci. Fenn., Ser. A, 15 : 321 (1921) Literature: Papong et al., 2007

1254. Trichothelium bipindense F.Schill., Hedwigia 67: 278 (1927)

Literature: Papong et al., 2007

1255. Trichothelium javanicum (F.Schill.) Vězda, Nova Hedwigia 58 : 139 (1994)

Literature: Papong et al., 2007

1256. Triclinum cinchonarum Fée, Essai Crypt. Écorc. (Paris): 148 (1825) [1824] Literature: Aptroot et al., 2007

1257. Triclinum sorediatum Aptroot \& Sparrius, in Aptroot et al., Fungal Diversity 24: 122 (2007)

Literature: Aptroot et al., 2007

1258. Trinathotrema lumbricoides (Sipman) Sipman \& Aptroot, in Lücking, et. al., Biblioth. Lichenol. 106: 199 (2011)

Literature: Vongshewarat et al., 1999; Wolseley et al., 2002 as Conotrema lumbricoides

1259. Trypethelium eluteriae Spreng., Anleit. Kenntn. Gew. 3: 350 (1804)

Literature: Luangsuphabool et al., 2016; Paulson 1930; Boonpragob et al., 1998; Wolseley et al., 2002; Aptroot and Lücking 2016

1260. Trypethelium epileucodes Nyl., Lich. Japon.: 116 (1890)

Literature: Aptroot et al., 2007 as Trypethelium subnitidiusculum; Aptroot and Lücking 2016

1261. Trypethelium platystomum Mont., Annls Sci. Nat., Bot., sér. 2. 19: 72 (1843)

Literature: Luangsuphabool et al., 2016; Aptroot and Lücking 2016

1262. Trypethelium subeluteriae Makhija \& Patw., Int. J. Mycol. Lichenol. 5: 245 (1992) Literature: Luangsuphabool et al., 2016

1263. Tylophoron moderatum Nyl., Bot. Zeitung 20: 279 (1862)

Literature: Wolseley and Aguirre-Hudson 1997a, 1997b; Wolseley et al., 2002

1264. Tylophoron protrudens Nyl., Ber. Tätigk. St. Gallischen Naturwiss. Ges.: 279 (1862) 
Literature: Aptroot et al., 2007

1265. Usnea australis Fr., Syst. Orb. Veg.: 282 (1825)

Literature: Satô 1962; Vainio 1921; Wolseley et al., 2002

1266. Usnea baileyi (Stirt.) Zahlbr., Denkschr. Kaiserl. Akad. Wiss., Wien. Math.Naturwiss. Kl. 83:182 (1909)

Literature: Vainio 1921 as Eumitria perrubescens; Satô 1962 as Usnea implicita; Yoshimura 1978 as Usnea baileyi subsp. baileyi; Wolseley et al., 2002

1267. Usnea baileyi subsp. chrysopora (Stein) Asahina, J. Jap. Bot. $42: 7$ (1967)

Literature: Yoshimura 1978; Wolseley et al., 2002

1268. Usnea baileyi subsp. septentrionalis Asahina, in Hara, Fl. E. Himalaya: 598 (1966) Literature: Yoshimura 1978; Wolseley et al., 2002

1269. Usnea cinchonarum (Fée) Zahlbr., Denkschr. Kaiserl. Akad. Wiss., Wien. Math.-Naturwiss. Kl. 83: 183-185 (1909)

Literature: Vainio 1921

1270. Usnea dasycera (Nyl.) Vain., Lich. Gen. Usnea Monogr.: 552 (1938) Literature: Vainio 1921 as Usnea perplexans f. dasycera; Wolseley et al., 2002

1271. Usnea endochroa var. papillata (Vain.) Zahlbr., Cat. Lich. Univ. 6: 564 (1930). Literature: Vainio 1921 as Eumitria endochroa var. papillata

1272. Usnea endorhodina (Vain.) Zahlbr., Cat. Lich. Univ. 6: 564 (1930). Literature: Vainio 1921 as Eumitria endorhodina; Wolseley et al., 2002

1273. Usnea florida (L.) Weber ex F.H.Wigg., Prim. Fl. Holsat. 2: 7 (1780) Literature: Vainio 1921

1274. Usnea florida var. subcomosa Vain., Philipp. J. Sci., C 4: 652 (1909). Literature: Wolseley et al., 2002

1275. Usnea himantodes Stirt., Scott. Naturalist 7:75 (1883)

Literature: Yoshimura 1978 as Usnea neoguineensis var. neoguineensis; Wolseley et al., 2002 as Usnea neoguineensis var. gracilior

1276. Usnea hossei Vain., Ann. Bot. Soc. Zool.-Bot. Fenn. "Vanamo" 1:34 (1921)

Literature: Satô 1962; Vainio 1921; Alava 1988; Wolseley et al., 2002

1277. Usnea leucospilodea Nyl., J. Linn. Soc., Bot. 20: 50 (1883)

Literature: Asahina 1969; Yoshimura 1978; Wolseley et al., 2002

1278. Usnea luridorufa Stirt., Scott. Naturalist 6: 104 (1881)

Literature: Wolseley et al., 2002

1279. Usnea neoguineensis var. neoguineensis Asahina, J. Jap. Bot. 43: 496 (1968)

Literature: Yoshimura 1978

1280. Usnea pectinata Stirt., Scott. Naturalist 1: 77 (1883)

Literature: Yoshimura 1978; Wolseley et al., 2002

1281. Usnea perplexans Stirt., Scott. Naturalist 6: 103 (1881)

Literature: Wolseley et al., 2002

1282. Usnea rubicunda Stirt., Naturalist 6:102 (1881)

Literature: Yoshimura 1978; Wolseley et al., 2002

1283. Vainionora flavidorufa (Hue) Papong \& Lumbsch, Lichenologist 43: 299-320 (2011) 
Literature: Vainio 1921; Wolseley et al., 2002 as Lecanora lividocarnea and Lecanora lividoglauca; Aptroot et al., 2007 as Lecanora flavidorufa; Papong and Lumbsch 2011

1284. Varicellaria velata (Turner) Schmitt \& Lumbsch, MycoKeys 4: 31 (2012)

Literature: Aptroot et al., 2007 as Pertusaria velata

1285. Verrucaria muralis Ach., Methodus (Acharius): 115 (1803)

Literature: Aptroot et al., 2007

1286. Vezdaea stipitata Poelt \& Döbbeler, Lichenologist 9: 170 (1977)

Literature: Aptroot et al., 2007

1287. Viridothelium virens (Tuck. ex Michener) Lücking, M.P. Nelsen \& Aptroot, in Lücking et al., Lichenologist 48: 759 (2016)

Literature: Luangsuphabool et al., 2017

1288. Wirthiotrema desquamans (Müll.Arg.) Lücking, in Sipman et al., Phytotaxa 55 (2012) Literature: Wolseley et al., 2002 as Myriotrema desquamans

1289. Wirthiotrema glaucopallens (Nyl.) Rivas Plata \& Kalb, in Rivas Plata et al., Lichenologist 42: 198 (2010)

Literature: Yoshimura 1978; Homchantara and Coppins 2002 as Myriotrema subanamaliense; Wolseley et al., 2002 as Thelotrema glaucopallens; Papong et al., 2010

1290. Xanthoparmelia australiensis (Cromb.) Hale, Phytologia 28: 486 (1974)

Literature: Paulson 1930 as Parmelia australiensis

1291. Xanthoparmelia congensis (J.Steiner) Hale, Phytologia 28: 486 (1974)

Literature: Pooprang et al., 1999; Moon et al., 2000a; Wolseley et al., 2002;

Louwhoff et al., 2012

1292. Xanthoparmelia mongeotina (Nyl.) D.J.Galloway, New Zealand J. Bot. 18: 538 (1981)

Literature: Pooprang et al., 1999; Wolseley et al., 2002; Louwhoff et al., 2012

\section{New combinations}

Lepra buloloensis (A.W.Archer, Elix \& Streimann) Schmitt \& Lumbsch, comb. nov. (MB 820271)

Basionym: Pertusaria buloloensis A.W. Archer, Mycotaxon 56: 388 (1995).

Lepra patellifera (A.W.Archer) Schmitt \& Lumbsch, comb. nov. (MB 820272)

Basionym: Pertusaria patellifera A.W. Archer, Mycotaxon 41: 237 (1991).

Lepra subventosa (Malme) Schmitt \& Lumbsch, comb. nov. (MB 820274)

Basionym: Pertusaria subventosa Malme, Ark. Bot. 28A: 7(1936). Syn.: Marfloraea subventosa (Malme) S.Y. Kondr., L. Lökös \& Hur, in Kondratyuk et al., Stud. Bot. Hungar. 46: 106 (2015).

\section{New records}

Acanthothecis salazinica S. Joshi \& Hur, Lichenologist 45: 600 (2013) 
Specimen examined: Thailand, Sukhothai province, Ban Dan Lan Hoi district, on trunk of Sindora siamensis Teijsm. ex Miq., in dry dipterocarp forest, $119 \mathrm{~m}$ alt., 2015, K. Vongshewarat \& S. Senglek RAMK— 30206 \& 30207.

Bactrospora metabola (Nyl.) Egea \& Torrente, Mycotaxon 53: 58 (1995)

Specimen examined: Thailand, Trat province, Koh chang district, Ban Slak Khok, on trunk of Rhizophora apiculata Bl. in mangrove, $0 \mathrm{~m}$ alt., 2013, M. Sodamuk \& N. Sriwongkorakot RAMK-23429.

Buellia parastata (Nyl.) Zahlbr., Cat. Lich. Univ. 7: 386 (1930)

Specimens examined: Thailand, Nakhon Ratchasima Province, Khao Yai National Park, on trunk of Lithocarpus gracilis (Korth.) Soepadmo in tropical rain forest, 816 $\mathrm{m}$ alt., 2000, K. Noicharoen RAMK-4072; on trunk of unidentified tree in secondary forest, 829 m alt., 1999, V. Sri-indrasutdhi RAMK- 4069; on trunk of unidentified tree in dry evergreen forest, $829 \mathrm{~m}$ alt., 1999, W. Polyiam RAMK— 4073.

Diploschistes cinereocaesius (Sw. ex Ach.) Vain., Ann, Acad. Sci. Fenn., ser. A 15(6): 172 (1921)

Specimen examined: Thailand, Mae Hong Son province, Khun Chae National Park, on soil in lower montane scrub, $1830 \mathrm{~m}$ alt., 2016, S. Senglek \& P. Fuangkeaw RAMK - 30208

Rolfidium coccocarpioides (Nyl.) Timdal, Op. bot. 110: 121 (1991)

Specimens examined: Thailand, Nakhon Ratchasima Province, Sakaerat Environmental Research Station, on sandstone in dry dipterocarp forest, $300 \mathrm{~m}$ alt., 2013, W. Polyiam \& N. Duangpui RAMK— 27954, $27955 \& 27956$.

Trapelia placodioides Coppins \& P. James, Lichenologist 16: 257 (1984)

Specimen examined: Thailand, Nakhon Ratchasima Province, Khao Yai National Park, Khao Khaeo on rock in lower montane forest, 1,393 m alt., 2001, K. Boonpragob RAMK- 23965

\section{Excluded species based on misidentifications (vouchers studied and reidentified)}

Graphis siamensis Vain., Ann. Soc. Zool. Bot. Fenn. "Vanamo" 1(3): 52 (1921) (Wolseley et al., 2002) = Diorygma hieroglyphicum (Pers.) Staiger \& Kalb, Biblioth. Lichenol. 85: 113 (2002)

Lecanora carpinea (Sriprang and Mongkolsuk 2010) = Lecanora tropica Zahlbr., Cat. Lich. Univ. 5: 589 (1928)

Platismatia glauca (Mongkolsuk et al., 1996) = Parmotrema praesorediosum $(\mathrm{Nyl}$.) Hale, Phytologia 28: 338 (1974)

Platismatia lacunosa (Mongkolsuk et al., 1996) = Parmotrema maclayanum (Müll.Arg.) Hale, Phytologia 28: 337 (1974)

Platismatia tuckermanii (Mongkolsuk et al., 1996) = Parmotrema explanatum (Hale) Hale, Phytologia 28: 337 (1974)

Roccella phycopsis (Paulson 1930) $=$ Roccella montagnei Bel., Voy. Indes Or. 2: 117 (1834) 


\section{Doubtful species}

Amandinea coniops (Meesim and Mongkolsuk 2010b)

Bacidia spadicea (Sriprang and Mongkolsuk 2010)

Biatora sphaeroides (Sriprang and Mongkolsuk 2010)

Biatora vernalis (Sriprang and Mongkolsuk 2010)

Buellia punctiformis (Vainio 1921)

Byssoloma meadii (Sriprang and Mongkolsuk 2010)

Caloplaca ferruginea (Sriprang and Mongkolsuk 2010)

Haematomma infuscum (Sriprang and Mongkolsuk 2010)

Lecidea inolescens Vain., Suom. Elain-ja Kasvit. Seuran Van. Julk. 1: 48 (1921) (Wolseley

et al., 2002) Type material unavailable

Physcia americana (Mongkolsuk et al., 1996)

Pyrrhospora gowardiana (Sriprang and Mongkolsuk 2010)

Ramboldia heterocarpa (Sriprang and Mongkolsuk 2010)

\section{Acknowledgements}

This work was financially supported by the National Council of Thailand (grant No. 183076).

\section{References}

Ahti T, Parnmen S, Mongkolsuk P (2008) Three new species of Cladonia from Thailand. Sauteria 15: 15-19.

Alava R (1988) Edvard August Vainio's Types in TUR-V and Other Herbaria, Publications from the Herbarium, 2. University of Turku, Turku, $513 \mathrm{pp}$.

Aptroot A (2006) Three new species of Lithothelium (Pyrenulaceae) from China and Thailand, with a revised world key and annotated list of species. Lichenologist 38: 541-548. https:// doi.org/10.1017/S0024282906005913

Aptroot A (2009) The lichen genus Traponora. Bibliotheca Lichenologica 100: 21-30.

Aptroot A, Lücking R (2016) A first collaborative attempt at a global revision of Trypetheliaceae (Ascomycota: Dothideomycetes: Trypetheliales). Lichenologist 48: 607-608. https:// doi.org/10.1017/s0024282916000517

Aptroot A, Lücking R, Sipman HJM, Umaña L, Chaves JL (2008) Pyrenocarpous lichens with bitunicate asci A first assessment of the lichen biodiversity inventory in Costa Rica. Bibliotheca Lichenologica 97: 1-162.

Aptroot A, Saipunkaew W, Sipman HJM, Sparrius LB, Wolseley PA (2007) New lichens from Thailand, mainly microlichens from Chiang Mai. Fungal Diversity 24: 75-134.

Aptroot A, Schumm F, Caceres MES (2012) Six new species of Pyrenula from the tropics. Lichenologist 44: 611-618. https://doi.org/10.1017/S0024282912000254 
Aptroot A, Thor G, Lücking R, Elix JA, Chaves JL (2009) The lichen genus Herpothallon reinstated. Bibliotheca Lichenologica 99: 19-66.

Asahina Y (1969) Lichenologische Notizen (215-216). Journal of Japanese Botany 44: 33-39.

Boonpragob K, Homchantara N, Coppins BJ, McCarthy PM, Wolseley PA (1998) An introduction to the lichen flora of Khao Yai National Park, Thailand. Botanical Journal of Scotland 50: 209-219. https://doi.org/10.1080/03746609808684918

Boonpragob K, Lumbsch HT, Sipman HJM (2010) 'Thelotremataceae workshop', Bangkok 10-15 March 2008. Lichenologist 42: 127. https://doi.org/10.1017/S0024282909990363 Brodo IM (2012) IAL7 - a perspective. IAL Newsletter 45: 8-9.

Buaruang K, Mongkolsuk P, Manoch L (2009) Morphology and anatomy of lichen family Parmeliaceae at Phu Hin Rongkla National Park. Journal of Microscopy Society of Thailand 23: $20-24$.

Buaruang K, Scharnagl K, Divakar P, Leavitt SD, Crespo A, Nash TH, Manoch L, Lücking R, Lumbsch HT (2015) Molecular data support Pseudoparmelia as a distinct lineage related to Relicina and Relicinopsis (Ascomycota, Lecanorales). Lichenologist 47: 43-49. https://doi. org/10.1017/S0024282914000577

Elix JA, Jariangprasert S, Archer AW (2008) New Pertusaria (lichenized Ascomycota) from Australia and Thailand. Telopea 12: 263-272. https://doi.org/10.7751/telopea20085815

Ertz D (2009) Revision of the corticolous Opegrapha species from the Paleotropics. Bibliotheca Lichenologica 102: 1-176.

Gueidan C, Aptroot A, Cáceres MEdS, Binh NQ (2016) Molecular phylogeny of the tropical lichen family Pyrenulaceae: contribution from dried herbarium specimens and FTA card samples. Mycological Progress 15: 7. https://doi.org/10.1007/s11557-015-1154-8

Hale ME (1965a) A monograph of Parmelia subgenus Amphigymnia. Contributions from the United States National Herbarium 36: 193-358.

Hale ME (1965b) Six new species of Parmelia from southeast Asia. Journal of Japanese Botany 40: 199-205.

Hale ME (1968) New Parmeliae from Southeast Asia. Journal of Japanese Botany 43: 324-327.

Hale ME (1975) A monograph of the lichen genus Relicina (Parmeliaceae). Smithsonian Contributions to Botany 26: 1-32. https://doi.org/10.5479/si.0081024X.26

Hale ME (1976a) A monograph of the lichen genus Bulbothrix Hale (Parmeliaceae). Smithsonian Contributions to Botany 32: 1-29. https://doi.org/10.5479/si.0081024x.32

Hale ME (1976b) A monograph of the lichen genus Parmelina Hale (Parmeliaceae). Smithsonian Contributions to Botany 33: 1-60. https://doi.org/10.5479/si.0081024x.33

Hale ME (1976c) A monograph of the lichen genus Pseudoparmelia Lynge (Parmeliaceae). Smithsonian Contributions to Botany 31: 1-62. https://doi.org/10.5962/bhl.title.122540

Homchantara N, Coppins BJ (2002) New species of the lichen family Thelotremataceae in SE Asia. Lichenologist 34: 113-140. https://doi.org/10.1006/lich.2002.0382

Jaklitsch WM, Baral HO, Lücking R, Lumbsch HT (2016) Ascomycota. In: Frey W (Ed) Syllabus of Plant Families - Adolf Engler's Syllabus der Pflanzenfamilien. Gebr. Borntraeger Verlagsbuchhandlung, Stuttgart, 1-150.

Jariangprasert S (2006) New taxa of the lichen genus Pertusaria (Ascomycota) from Thailand. Mycotaxon 96: 109-121. 
Jariangprasert S, Anusarnsunthorn V (2005) Additional new taxa in the lichen genus Pertusaria (lichenized Ascomycota) from Thailand. Mycotaxon 91: 279-292.

Jariangprasert S, Archer AW, Anusarnsunthorn V (2004) Further new species in the genus Pertusaria (lichenized Ascomycota) from Thailand. Mycotaxon 89: 123-129.

Jariangprasert S, Archer AW, Elix JA, Anusarnsunthorn V (2002) Two new species in the lichen genus Pertusaria (Ascomycotina) from Thailand. Mycotaxon 83: 353-356.

Jariangprasert S, Archer AW, Elix JA, Anusarnsunthorn V (2003) New taxa in the lichen genus Pertusaria (lichenized ascomycotina) from Thailand. Mycotaxon 85: 289-295.

Jørgensen PM (2000) Notes on some East-Asian species of the lichen genus Fuscopannaria. Journal of the Hattori Botanical Laboratory 89: 247-259.

Jørgensen PM, Wolseley PA (2009) Leioderma sorediatum D. J. Galloway \& P. M. Jørg. discovered in Thailand, with a note on the world distribution of Erioderma mollissimum (Sampaio) Du Rietz. Lichenologist 41: 315-316. https://doi.org/10.1017/S0024282909008482

Kalb J, Boonpragob K, Kalb K (2016a) New Coenogonium species (Ostropales: Coenogoniaceae) from Thailand, new reports and a revised key to the species occurring in the country. Phytotaxa 283: 101-122. https://doi.org/10.11646/phytotaxa.283.2.1

Kalb J, Polyiam W, Plata ER, Bawingan PA, Kalb K, Lücking R (2016b) 'Missing links' alive? Novel taxa represent morphological transitions between distinctive phenotypes among extant Graphidaceae (lichenized Ascomycota: Ostropales). Phytotaxa 268: 110-122. https:// doi.org/10.11646/phytotaxa.268.2.2

Kalb K (2009) New taxa and new records of thelotremoid Graphidaceae. Herzogia 22: 17- 42.

Kalb K, Archer AW, Sutjaritturakan J, Boonpragob K (2009a) New or otherwise interesting lichens V. Bibliotheca Lichenologica 99: 225-246.

Kalb K, Buaruang K, Mongkolsuk P, Boonpragob K (2012) New or otherwise interesting lichens. VI, including a lichenicolous fungus. Phytotaxa 42: 35-47. https://doi.org/10.11646/phytotaxa.42.1.5

Kalb K, Buaruang K, Papong K, Boonpragob K (2009b) New or otherwise interesting lichens from the tropics, including the lichen genus Ramboldia in Thailand. Mycotaxon 110: 109-123. https://doi.org/10.5248/110.109

Kalb K, Plata ER, Lücking R, Lumbsch TH (2011) The phylogenetic position of Malmidea, a new genus for Lecidea piperis- and Lecanora granifera-groups (Lecanorales, Malmideaceae), inferred from neclear and mitochondrial ribosomal DNA sequences, with special reference to Thai species. Bibliotheca Lichenologica 106: 143-168.

Kantvilas G, Papong K, Lumbsch HT (2010) Further observations on the genus Maronina, with the description of two new taxa from Thailand. Lichenologist 42: 557-561. https:// doi.org/10.1017/S0024282910000174

Kirika PM, Divarkar PK, Leavitt SD, Buaruang K, Crespo A, Mugambi G, Gatheri GW, Lumbsch HT (2017) The genus Relicinopsis is nested within Relicina (Parmeliaceae, Ascomycota). Lichenologist. [in press]

Kraichak E, Parnmen S, Lücking R, Lumbsch HT (2013) Gintarasia and Xalocoa, two new genera to accommodate temperate to subtropical species in the predominantly tropical Graphidaceae (Ostropales, Ascomycota). Australian Systematic Botany 26: 466-474. 
Kraichak E, Parnmen S, Lücking R, Rivas-Plata E, Aptroot A, Caceres MES, Ertz D, Mangold A, Mercado-Diaz JA, Papong K, Broeck DVD, Weerakoon G, Lumbsch HT (2014) Revisiting the phylogeny of Ocellularieae, the second largest tribe within Graphidaceae (Lichenized Ascomycota: Ostropales). Phytotaxa 189: 52-81. https://doi.org/10.11646/ phytotaxa.189.1.6

Kurokawa S (1998) A new record and a new species in Myelochroa (Parmeliaceae). Journal of Japanese Botany 73: 12-14.

Lai MJ, Elix JA (2002) A new species of Cetreliopsis (Ascomycotina, Parmeliaceae) from Thailand. Mycotaxon 84: 355-360.

Louwhoff SHJJ, Nimis PL, Wolseley PA, Saipunkaew W (2012) A key to the parmelioid lichens of northern Thailand.

Luangsuphabool T, Piapukiew J, Parnmen S, Nelsen MP, Lumbsch HT, Sangvichien E (2016) Diversity of the Trypethelium eluteriae group in Thailand (Ascomycota, Trypetheliales). Lichenologist 48: 53-60. https://doi.org/10.1017/S0024282915000444

Luangsuphabool T, Piapukiew J, Lumbsch HT, Sangvichien E (2017) First record of Viridothelium virens (Trypetheliales, Ascomycota) in the Southeast Asian tropics. Herzogia (in review).

Lücking R, Hodkinson BP, Leavitt SD (2016) The 2016 classification of lichenized fungi in the Ascomycota and Basidiomycota - Approaching one thousand genera. Bryologist 119: 361-416. https://doi.org/10.1639/0007-2745-119.4.361

Lücking R, Mangold A, Rivas Plata E, Parnmen S, Kraichak E, Lumbsch TH (2015) Morphology-based phylogenetic binning to assess a taxonomic challenge: a case study in Graphidaceae (Ascomycota) requires a new generic name for the widespread Leptotrema wirthii. Botanical Journal of the Linnean Society 436: 436-443. https://doi.org/10.1111/boj.12327

Lücking R, Sutjaritturakan J, Kalb K (2012) Validation of three species names and description of a new species in the genus Graphis (Ascomycota: Ostropales: Graphidaceae). Lichenologist 44: 391-394. https://doi.org/10.1017/s0024282911000855

Lumbsch HT (2000) Phylogeny of filamentous ascomycetes. Naturwissenschaften 87: 335-342. https://doi.org/10.1007/s001140050736

Lumbsch HT, Ahti T, Altermann S, De Paz GA, Aptroot A, Arup U, Pena AB, Bawingan PA, Benatti MN, Betancourt L, Bjork CR, Boonpragob K, Brand M, Bungartz F, Caceres MES, Candan M, Chaves JL, Clerc P, Common R, Coppins BJ, Crespo A, Dal-Forno M, Divakar PK, Duya MV, Elix JA, Elvebakk A, Fankhauser JD, Farkas E, Ferraro LI, Fischer E, Galloway DJ, Gaya E, Giralt M, Goward T, Grube M, Hafellner J, Hernandez JE, Campos M, Kalb K, Karnefelt I, Kantvilas G, Killmann D, Kirika P, Knudsen K, Komposch H, Kondratyuk S, Lawrey JD, Mangold A, Marcelli MP, Mccune B, Messuti MI, Michlig A, Gonzalez RM, Moncada B, Naikatini A, Nelsen MP, Ovstedal DO, Palice Z, Papong K, Parnmen S, Perez-Ortega S, Printzen C, Rico VJ, Plata ER, Robayo J, Rosabal D, Ruprecht U, Allen NS, Sancho L, De Jesus LS, Vieira TS, Schultz M, Seaward MRD, Serusiaux E, Schmitt I, Sipman HJM, Sohrabi M, Sochting U, Sogaard MZ, Sparrius LB, Spielmann A, Spribille T, Sutjaritturakan J, Thammathaworn A, Thell A, Thor G, Thus H, Timdal E, Truong C, Turk R, Tenorio LU, Upreti DK, Van den Boom P, Rebuelta MV, Wedin M, Will-Wolf S, Wirth V, Wirtz N, Yahr R, Yeshitela K, Ziemmeck F, Wheeler T, Lücking R 
(2011) One hundred new species of lichenized fungi: a signature of undiscovered global diversity. Phytotaxa 18: 1-127. https://doi.org/10.11646/phytotaxa.18.1.1

Lumbsch HT, Huhndorf SM (2010) Myconet Volume 14. Part One. Outline of Ascomycota 2009. Fieldiana (Life and Earth Sciences) 1: 1-42. https://doi.org/10.3158/1557.1

Lumbsch HT, Parnmen S, Kraichak E, Papong KB, Lücking R (2014) High frequency of character transformations is phylogenetically structured within the lichenized fungal family Graphidaceae (Ascomycota: Ostropales). Systematics and Biodiversity 12: 271-291. https:// doi.org/10.1080/14772000.2014.905506

McCarthy PM (1999) Three new species of Porina (Trichotheliaceae) from Thailand. Lichenologist 31: 239-246. https://doi.org/10.1017/S0024282999000341

Medeiros ID, Kraichak E, Lücking R, Mangold A, Lumbsch HT (2017) Assembling a taxonomic monograph of tribe Wirthiotremateae (Lichenized Ascomycota: Ostropales: Graphidaceae). Fieldiana, Life and Earth Sciences 9: 1-31.

Meesim S, Mongkolsuk P (2010a) Foliose lichens of family Physciaceae at Phu Luang Wildlife Sanctuary, Loei province. Thai Journal of Botany 2: 55-64.

Meesim S, Mongkolsuk P (2010b) Study on the crustose lichens of family Physciaceae in Thailand. Thai Journal of Botany 2: 65-72.

Mongkolsuk P, Buaruang K, Vongshewarat K, Doodurm C, Dangphui N, Poengsuangnoen V, Sriprang V, Meesim S, Parnmen S, Sodamuk M (2013) Biodiversity of lichen in Phu Luang Wildlife Sanctuary, Loei province. Thai Journal of Botany 5: 19-30.

Mongkolsuk P, Meesim S (2012) Biodiversity of lichen family Physciaceae (Lichenized Ascomycota) in Phu Luang Wildlife Sanctuary, Loei Province, Thailand. Ramkhamhaeng University Reseach Journal of Sciences and Technology 15: 69-83.

Mongkolsuk P, Meesim S, Poengsungnoen V, Buaruang K, Schumm F, Kalb K (2015) The lichen family Physciaceae in Thailand - II. The genus Heterodermia sensu lato. Phytotaxa 235: 1-66. https://doi.org/10.11646/phytotaxa.235.1.1

Mongkolsuk P, Meesim S, Poengsungnoen V, Kalb K (2012) The lichen family Physciaceae in Thailand - I. The genus Pyxine. Phytotaxa 59: 32-54. https://doi.org/10.11646/phytotaxa.59.1.2

Mongkolsuk P, Poengsungnoen V (2012) The lichen family Graphidaceae, Natural Art. Nobel Print, Phayathy, Bangkok, 195 pp.

Moon KH, Kurokawa S, Kashiwadani H (2000a) A list of Thailand species of Parmelia (sens. lat.) preserved in the National Science Museum. In: Matsuura KE (Ed) The first and second symposia on collection building and natural history studies in Asia. National Science Museum Monographs, 97-106.

Moon KH, Kurokawa S, Kashiwadani H (2000b) A new species of Hypotrachyna (Lichenized Ascomycetes, Parmeliaceae) from Thailand. Bulletin of the National Science Museum (Tokyo), Series B (Botany) 26: 135-138.

Nakanishi M, Kashiwadani H, Moon KH (2001) Notes on the genera Graphina and Graphis (Graphidaceae) in Thailand. Bulletin of the National Science Museum (Tokyo), Series B (Botany) 27: 47-55.

Naksuwankul K, Kraichak E, Parnmen S, Lücking R, Lumbsch HT (2016) Five new species of Graphidaceae (Ascomycota, Ostropales) from Thailand. MycoKeys 17: 47-63. https://doi. org/10.3897/mycokeys.17.10512 
Nelsen MP, Lücking R, Plata ER, Mbatchou JS (2010) Heiomasia, a new genus in the lichenforming family Graphidaceae (Ascomycota: Lecanoromycetes: Ostropales) with disjunct distribution in Southeastern North America and Southeast Asia. Bryologist 113: 742-751. https://doi.org/10.1639/0007-2745-113.4.742

Neuwirth G, Stocker-Wörgötter E, Boonpragob K, Saipunkaew W (2014) Coenogonium coronatum (Ostropales: Coenogoniaceae), a new foliicolous species from Thailand, ecological aspects and a key to the species occurring in the country. Bryologist 117: 161-164. https:// doi.org/10.1639/0007-2745-117.2.161

Noicharoen K, Polyiam W, Boonpragob K, Elix JA, Wolseley PA (2003) New species of Parmotrema and Relicina (Ascomycota, Parmeliaceae) from Thailand. Mycotaxon 85: 325-330.

Ohmura Y, Frisch A, Lendemer JC, Nakanishi M (2016) Lichens of Mikura-jima Island, Izu Islands, Central Japan. Bull Natl Mus Nat Sci, Ser B 42: 1-18.

Orange A, Wolseley PA (2005) Two new thamnolic acid-containing Lepraria species from Thailand. Lichenologist 37: 247-250. https://doi.org/10.1017/S0024282905015136

Papong K, Boonpragob K, Lücking R (2007) New species and new records of foliicolous lichens from Thailand. Lichenologist 39: 47-56. https://doi.org/10.1017/S0024282906006104

Papong K, Boonpragob K, Lumbsch HT (2009a) Additional lichen records from Thailand 1. Loxospora lecanoriformis (Sarrameanaceae). Australasian Lichenology 65: 50-53.

Papong K, Boonpragob K, Lumbsch HT (2011a) A new species and new records of Lecanora (Lecanoraceae, Ascomycota) from south-east Asia. Lichenologist 43: 47-50. https://doi. org/10.1017/S0024282910000496

Papong K, Boonpragob K, Mangold A, Divakar PK, Lumbsch TH (2010) Thelotremoid lichen species recently described from Thailand: a re-evaluation. Lichenologist 42: 131-137. https://doi.org/10.1017/S0024282909990405

Papong K, Kantvilas G, Lumbsch TH (2011b) Morphological and molecular evidence places Maronina into synonymy with Protoparmelia (Ascomycota: Lecanorales). Lichenologist 43: 561-567. https://doi.org/10.1017/S0024282911000284

Papong K, Lücking R, Thammathaworn A, Boonpragob K (2009b) Four new taxa of Chroodiscus (thelotremoid Graphidaceae) from Southeast Asia. Bryologist 112: 152-163. https:/doi. org/10.1639/0007-2745-112.1.152

Papong K, Lumbsch TH (2011) A taxonomic survey of Lecanora sensu stricto in Thailand (Lecanoraceae; Ascomycota). Lichenologist 43: 299-320. https://doi.org/10.1017/S0024282911000247

Papong K, Thammathaworn A, Boonpragob K (2008) Rolueckia (Ostropales: Gomphillaceae), a new genus of foliicolous lichens. Nova Hedwigia 86: 201-208. https://doi.org/10.1127/0029-5035/2008/0086-0201

Papong KB, Mangold A, Lücking R, Lumbsch TH (2014) New species and new records of thelotremoid Graphidaceae (Ascomycota: Ostropales) from Thailand. Phytotaxa 189: 232-244. https://doi.org/10.11646/phytotaxa.189.1.16

Parnmen S, Lücking R, Lumbsch HT (2012) Phylogenetic classification at generic level in the absence of distinct phylogenetic patterns of phenotypical variation: A case study in Graphidaceae (Ascomycota). PLoS One 7(12): e51392. https://doi.org/10.1371/journal.pone.0051392

Parnmen S, Rangsiruji A, Mongkolsuk P, Ahti T (2008) Phylogenetics of lichens in the genus Cladonia (Cladoniaceae) in northern and northeastern Thailand. Sauteria 15: 385-402. 
Paulson R (1930) Lichens from Kaw Tao, an island in the Gulf of Siam. Journal of the Siam Society, Natural History Supplement 8: 99-101.

Pitakpong A, Kraichak E, Papong KB, Muangsan N, Suwanwaree P, Lumbsch HT, Lücking R (2015) New species and records of the lichen genus Graphis (Graphidaceae, Ascomycota) from Thailand. Lichenologist 47: 335-342. https://doi.org/10.1017/S0024282915000213 Poengsungnoen V, Manoch L, Mongkolsuk P, Boonpragob K, Parnmen S, Lücking R, Tehler A, Lumbsch TH (2014a) Phylogenetic analysis reveals two morphologically unique new species in the genera Astrochapsa and Nitidochapsa (lichenized Ascomycota: Graphidaceae). Phytotaxa 189: 268-281. https://doi.org/10.11646/phytotaxa.189.1.19

Poengsungnoen V, Manoch L, Mongkolsuk P, Kalb K (2014b) New species of Graphidaceae from Loei Province, Thailand. Phytotaxa 189: 255-267. https://doi.org/10.11646/phytotaxa.189.1.18

Poengsungnoen V, Mongkolsuk P, Boonpragob K, Manoch L (2010) Diversity of the lichen family Graphidaceae in Phu Luang Wildlife Sanctuary, Loei province. Thai Journal of Botany 2: 73-79. https://doi.org/10.1007/978-3-642-02167-1_10

Pooprang T, Boonpragob K, Elix JA (1999) New species and new records in the lichen family Parmeliaceae (Ascomycotina) from Thailand. Mycotaxon 71: 111-127.

Printzen C (2010) Lichen Systematics: The role of morphological and molecular data to reconstruct phylogenetic relationships. Progress in Botany 71: 233-275.

Rangsiruji A, Boonpragob K, Mongkolsuk P, Sodamuk M, Buaruang K, Binchai S, Lumbsch HT, Parnmen S (2016) Diversity and phylogenetic survey of cyanobacterial lichens (Collematineae, Ascomycota) in mangrove forests of eastern Thailand. Bryologist 119: 123-130. https://doi.org/10.1639/0007-2745-119.2.123

Rivas Plata E, Lücking R, Lumbsch TH (2012a) Molecular phylogeny and systematics of the Ocellularia clade (Ascomycota: Ostropales: Graphidaceae). Taxon 61: 1161-1179.

Rivas Plata E, Lücking R, Lumbsch TH (2012b) A new classification for the family Graphidaceae (Ascomycota: Lecanoromycetes: Ostropales). Fungal Diversity 52: 107-121. https://doi.org/10.1007/s13225-011-0135-8

Saipunkaew W, Wolseley PA, Chimonides PJ, Boonpragob K (2007) Epiphytic macrolichens as indicators of environmental alteration in northern Thailand. Environmental Pollution 146: 366-374. https://doi.org/10.1016/j.envpol.2006.03.044

Satô M (1962) Lichens of Doi Inthanon and Doi Suthep, northwestern Thailand. Nature and Life in Southeast Asia 2: 1-4.

Schumm F, Aptroot A (2012) A microscopical atlas of some tropical lichens from SE-Asia (Thailand, Cambodia, Philippines, Vietnam). Privately published, Norderstedt, 881 pp.

Sodamuk M, Boonpragob K, Mongkolsuk P, Tehler A, Leavitt SD, Thorsten Lumbsch HT (2017) Kalbionora palaeotropica, a new genus and species from coastal forests in Southeast Asia and Australia (Malmideaceae, Ascomycota). MycoKeys 22: 15-25. https://doi. org/10.3897/mycokeys.22.12528

Sparrius LB, Saipunkaew W (2005) Cryptothecia punctosorediata, a new species from northern Thailand. Lichenologist 37: 507-509. https://doi.org/10.1017/S0024282905015495 
Sparrius LB, Saipunkaew W, Wolseley PA, Aptroot A (2006) New species of Bactrospora, Enterographa, Graphidastra and Lecanographa from northern Thailand and Vietnam. Lichenologist 38: 27-36. https://doi.org/10.1017/S0024282905005414

Sriprang V, Mongkolsuk P (2010) Discolichens at Phu Luang Wildlife Sanctuary, Loei province. Thai Journal of Botany 2: 47-53.

Sutjaritturakan J, Kalb K (2015) Ocellularia (Ascomycota: Ostropales) - three new species, a new record and a key for all species so far recorded for Thailand. Herzogia 28: 545-555. https://doi.org/10.13158/heia.28.2.2015.545

Sutjaritturakan J, Saipunkaew W, Boonpragob K, Kalb K (2014) New species of Graphidaceae (Ostropales, Lecanoromycetes) from southern Thailand. Phytotaxa 189: 312-324. https:// doi.org/10.11646/phytotaxa.189.1.22

Takhtajan A (1986) Floristic regions of the world. University of California Press, Berkeley, 522 pp. Vainio EA (1907) Lichenes novi rarioresque, IV. Hedwigia 46: 168-181.

Vainio EA (1909) Lichenes. In Schmidt J (Ed.) Flora of Koh Chang; Contribution to the knowledge of the vegetation of the Gulf of Siam. Botanisk Tidsskrift 29: 104-151.

Vainio EA (1921) Lichenes in summo monte Doi Sutep (circ. 1675 m.s.m.) in Siam boreali anno 1904 a D:re C.C. Hosseo collecti. Annales Societatis Zoologicae-Botanicae Fennicae "Vanamo" 1:33-55.

van den Boom PPG, Giralt M, Moberg R (2014) Lichen diversity of Physciaceae from Thailand. Graphis Scripta 26: 54-62.

Vongshewarat K, McCarthy PM, Mongkolsuk P, Boonpragob K (1999) Additions to the lichen flora of Thailand. Mycotaxon 70: 227-236.

Wolseley PA, Aguirre-Hudson B (1995) Key to lichen genera in Thailand with special reference to epiphytic taxa, part I: Macrolichens. Natural History Bulletin of the Siam Society 43: 303-335.

Wolseley PA, Aguirre-Hudson B (1997a) The ecology and distribution of lichens in tropical deciduous and evergreen forests of northern Thailand. Journal of Biogeography 24: 327-343. https://doi.org/10.1046/j.1365-2699.1997.00124.x

Wolseley PA, Aguirre-Hudson B (1997b) Fire in tropical dry forests: corticolous lichens as indicators of recent ecological changes in Thailand. Journal of Biogeography 24: 345-362. https://doi.org/10.1046/j.1365-2699.1997.00125.x

Wolseley PA, Aguirre-Hudson B, McCarthy PM (2002) Catalogue of the lichens of Thailand. Bulletin of the Natural History Museum, London (Botany) 32: 13-59. https://doi. org/10.1017/s0968044602000038

Wolseley PA, Moncrieff C, Aguirre-Hudson B (1994) Lichens as indicators of environmental stability and change in the tropical forests of Northern Thailand. Global Ecology and Biolography Letters 4: 116-123. https://doi.org/10.2307/2997437

Yoshimura I (1978) Some lichens of Thailand collected by Danish botanists, 1958-68. Bulletin of the Kochi Gakuen Junior College 9: 35-40. 